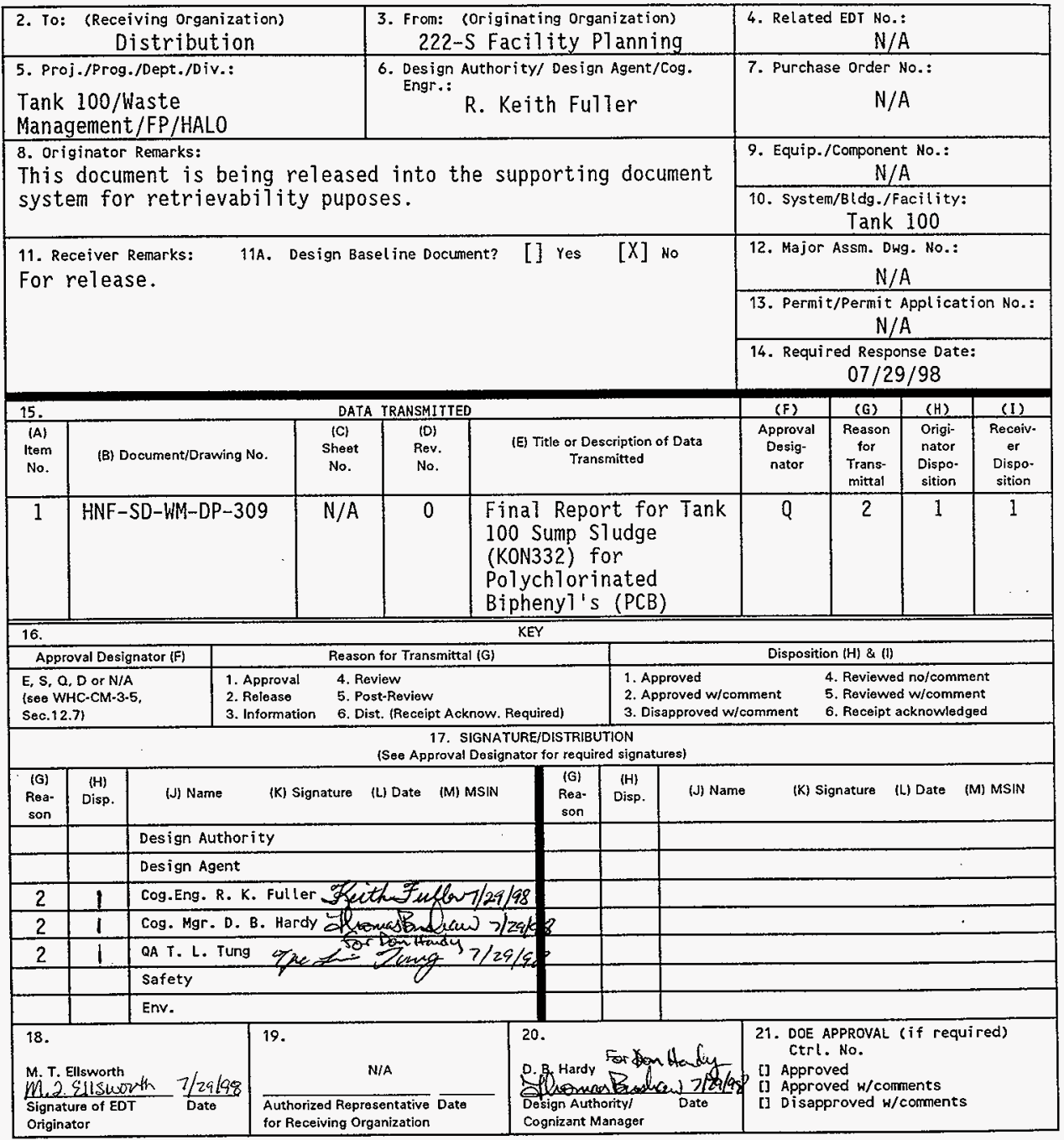




\title{
Final Report for Tank 100 Sump Sludge (KON332) for Polychlorinated Biphenyl's (PCB)
}

\author{
R. Keith Fuller \\ Waste Management of Hanford, Inc., Richland, WA 99352 \\ U.S. Department of Energy Contract DE-AC06-96RL13200
}
EDT/ECN: EDT-623162 UC: 2070
Org Code: 31132 Charge Code: KNOEO
B\&R Code: EW 3120074 Tota1 Pages: 164

Key Words: Final Report, Tank 100, 100, Sump, Sludge, K0N332, Polychlorinated Biphenyls, PCB

Abstract: N/A

TRADEMARK DISCLAIMER. Reference herein to any specific commercial product, process, or service by trade name, trademark, manufacturer, or otherwise, does not necessarily constitute or imply its endorsement, recommendation, or favoring by the United States Government or any agency thereof or its contractors or subcontractors.

Printed in the United States of America. To obtain copies of this document, contact: Document Control Services, P.0. Box 950, Mailstop H6-08, Richland WA 99352, Phone (509) 372-2420; Fax (509) 376-4989.
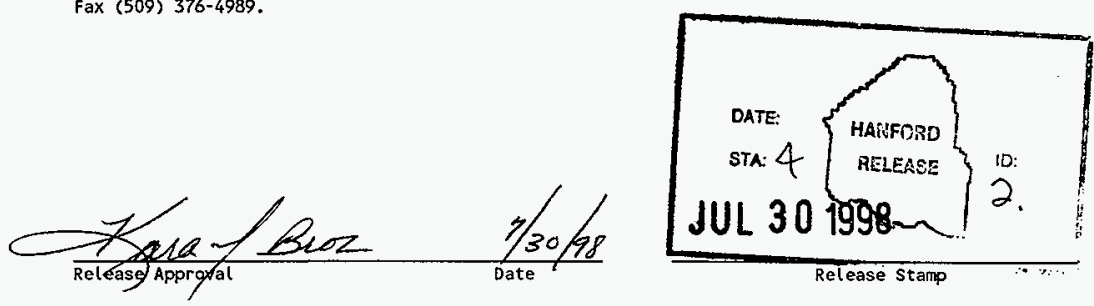

Approved for Public Release 
HNF-SD-WM-DP-309, REV. 0

HANFORD ANALYTICAL LABORATORY OPERATIONS

\title{
FINAL REPORT FOR \\ TANK 100 SUMP SLUDGE (KON332) \\ FOR POLYCHLORINATED BIPHENYL'S (PCB)
}

\author{
Project Coordinator: R. Keith Fuller \\ Prepared for the U.S. Department of Energy \\ office of Environmental Restoration \\ and Waste Management \\ by \\ 222-S Laboratory \\ Waste Management of Hanford, Inc. \\ P. 0. Box 700 \\ Richland, Washington
}


HNF-SD-HM-DP-309, REV. 0

THIS PAGE INTENTIONALLY LEFT BLANK 
HNF-SD-WM-DP-309, REV. 0

TABLE OF CONTENTS

Introduction (Attachment 1) . . . . . . . . . . 1 Narrative and Data Summary (Attachment 2) . . . . . . . . . 4 Sample Breakdown Diagrams (Attachment 3) . . . . . . . . 15 Chain of Custody and Request for Sample Analysis (Attachment 4) . . . . 18 222-S Extraction Bench Sheet (Attachment 5) . . . . . . . . . 22 PCB Raw Data (Attachment 6) . . . . . . . . . . . 25 This document consists of pages 1 through 160 . Pages $i i, 2,5,16,19,23$ and 26 were intentionally left blank. 
HNF-SD-WM-DP-309, REV. 0

ATTACHMENT 1

INTRODUCTION 
HNF-SD-HM-DP-309, REV. 0

THIS PAGE INTENTIONALLY LEFT BLANK 


\section{HNF-SD-WM-DP-309 REV. 0}

Final Report for Tank 100 Sump Sludge (KON332) for Polychlorinated Biphenyl's (PCB)

\section{Sample Receipt}

Sample KON332 was received from Tank 100-Sump (WESF) on May 18, 1998. The laboratory number issued for this sample is $\mathrm{S} 98 \mathrm{~B} 000207$ as shown on the Request for Sample Analysis (RSA) form (Attachment 4).

The sample breakdown diagram (Attachment 3 ) provides a cross-reference of customer sample identification to the laboratory identification number. Attachment 4 provides copies of the Request for Sample Analysis (RSA) and Chain of Custody (COC) forms.

\section{Sample Description, Handling, and Preparation}

- The sample was received in the laboratory in a $125-\mathrm{ml}$ polybottle. Breakdown and subsampling was performed on June 6, 1998.

\section{Analytical Results}

Polychlorinated Biphenyl (PCB)

PCB analysis was performed on the wet sludge. The laboratory sample number for the wet sludge is S98B000220. A discussion of the results is presented in Attachment 2.

The 222-S extraction bench sheets are presented in Attachment 5. The PCB raw data is presented in Attachment 6. 
HNF-SD-HM-DP-309, REV. 0

ATTACHMENT 2

NARRATIVE AND DATA SUMMARY

4 
HNF-SD-WM-DP-309, REV. 0

THIS PAGE INTENTIONALLY LEFT BLANK 


\title{
HNF-SD-WIM-DP-309 REV. 0
}

POLYCHLORINATED BIPHENYLS (PCB) ANALYSIS REPORT

FOR SOLID SAMPLE FROM WESF TANK 100 SUMP

\author{
222-S Laboratory \\ July 1998 \\ submitted by
}

GA Ross

Waste Management Federal Services of Hanford 


\section{POLYCHLORINATED BIPHENYLS (PCB) ANALYSIS REPORT FOR SOLID SAMPLE FROM WESF TANK 100 SUMP}

\section{SAMPLE ANALYSIS REPORTED}

One waste sample with centrifuged solids was obtained from the 222-S Hot Cells. The sample was received in a plastic bottle from the Waste Encapsulation and Storage Facility (WESF) and centrifuged. The centrifuged solid was then obtained from the hot cell in a glass jar with Teflon Iid, was analyzed (with duplicate, matrix spike, matrix spike duplicate, method blank and Taboratory control sample) for PCBs as Aroclor mixtures by the Direct Analysis Team. A soxhlet extraction procedure was used to extract the Aroclors from the sample. Analysis was performed using dual column confirmation gas chromatography/ electron capture detection (GC/ECD). Extraction closely follows SW-846 method $3540 \mathrm{C}$, and the analysis follows SW846 method 8082 .

SAMPLE DESCRIPTION, HANDLING, AND PREPARATION

The sample was received in a plastic bottle and introduced to the 222-S Hotcells. The solids of the sample were collected by centrifugation and transferred to a glass wide-mouth jar with Teflon Tid. This sample, S98B000220, consisting of centrifuged solids from a jar of TK-100 sludge, was analyzed and reported on a post centrifugation basis. The sample was grey in color and had the consistency of putty. After one minute of shaking and one minute of stirring, four 1-gram aliquots were taken from the parent sample jar for analysis. Extraction of the samples were started on June 15, 1998 in a radiological fume hood. Due to duplicate and other QC failures with the first set of extractions, extraction of four 2-2.5 gram aliquots of the sample was started on June 19, 1998. The results of the second set of sample extractions are reported here.

Preparation Procedure: LA-523-138 Rev. A2, Soxhlet Extraction of Solid Samples for Semivolatile and/or Pesticide and/or PCB Analysis, Waste Management Federal Services of Hanford, Inc. for Fluor Daniel Hanford, Inc., Richland, Washington.

Preparation Location: Room 4P, 222-S Laboratory, 200W Area, Hanford Site

Preparation Type: $\quad$ Soxhlet Extraction using methylene chloride, KudernaDanish concentration with solvent exchange to hexane and cleanup by sulfuric acid.

Sample Extract Storage: 4 degrees Celsius in darkness

1 Teflon is a trademark of E.I. duPont de Nemours \& Company, Incorporated.

2 Aroclor is a trademark of the Monsanto Company 


\section{HNF-SD-WM-DP-309 REV. 0}

ANALYSIS METHOD

GC/ECD Procedure: $\quad$ LA-523-136 Rev. Al, Gas Chromatograph Analysis of Pesticides and PCBS - SW-846, Waste Management Federal Services of Hanford, Inc. for Fluor Daniel Hanford, Inc., Richland, Washington.

GC/ECD instrumentation: HP-5890 Series II gas chromatograph with dual on-column injection, XTI-5 and DB-608 columns, and dual electron capture (EC) Detectors, (property number WC 44940).

Location: $\quad$ Room 4TUV, 222-S Laboratory

Due to the high concentrations of PCBs present, the extracts were analyzed on May 30, 1998 with a dilution. The (100X) dilution of the sample and sample duplicate was accomplished by diluting $10.0 \mu \mathrm{L}$ of the extract with $990 \mu \mathrm{L}$ of hexane. The laboratory control sample, matrix spike and matrix spike duplicate were accomplished by adding 200-400 $\mu \mathrm{L}$ of $40 \mu \mathrm{g} / \mathrm{mL}$ Aroclor 1254 standard before dilution.

QUALITY CONTROL

Due to radiological issues the extraction portion of the procedure was scaled down from SW-846 method 3540C. Appendix A of LA-523-138 details the deviations to method $3540 \mathrm{C}$ that are contained in the procedure. The analysis follows SW-846 method 8082. Deviations from method 8082 are detailed in appendix D of LA-523-136. Nominal1y, two grams of sample was extracted per analytical result.

For the initial calibration, five point calibrations were run for the following Aroclors: 1016, 1221, 1248, 1254, and 1260. Concentration ranges went from 20 to $400 \mathrm{ng} / \mathrm{mL}$, or 40 to $800 \mathrm{ng} / \mathrm{mL}$ for 1221 . Three point calibrations were performed for Aroctors 1232, and 1242. The sample analysis was bracketed with mid-level Aroclor 1254 and Aroclor 1221 standards. An instrument blank, method blank and LCS were analyzed during the sequence of sample analysis. A trip blank was not received with the sample.

Quantitation was performed using LA-523-136 option 1 (three peak) for Aroclors. A primary column and the secondary column was used to confirm identification. A detailed description of the specific procedural requirements is available in the analytical procedure LA-523-136. Laboratory bench sheets, data, chromatograms, and reports are maintained in room 25, 2704-S at the 222-S Laboratory. The initial calibration information and data is located in the attached initial calibration report or on the HP UNIX computer system, Chemsysl.

Table I summarizes the QC requirements and adherence. 
Table I.

\begin{tabular}{|c|c|}
\hline QC Parameter & Comments \\
\hline Target Compounds & $\begin{array}{l}\text { Detection levels are based on sample size and the } \\
\text { determination of Method Detection Limits (MDLs) from } \\
\text { multiple injections of a low standard. Requirements } \\
\text { were met. }\end{array}$ \\
\hline Surrogate Recoveries & $\begin{array}{l}\text { Surrogate spiking was performed on all samples using } \\
\text { Tetrachloro-m-xylene (TCX) and Decachlorobiphenyl } \\
\text { (DCB). A summary of the surrogate recoveries is } \\
\text { provided in Table II. A high outside bias was } \\
\text { observed for DCB due to coelution of a peak that } \\
\text { precedes the DCB in the sample chromatograms. All } \\
\text { of the primary surrogate (TCX) recoveries were } \\
\text { acceptable (see discussion). }\end{array}$ \\
\hline $\begin{array}{l}\text { Matrix Spike } \\
\text { Recovery }\end{array}$ & $\begin{array}{l}\text { A matrix spike (MS) and matrix spike duplicate (MSD) } \\
\text { using Aroclor } 1254 \text { was performed. MS/MSD recoveries } \\
\text { were } 51.1 \% \text {, and } 78.4 \% \text { which is acceptable (see Table } \\
\text { III). }\end{array}$ \\
\hline Method Blank Summary & A method blank was extracted and met requirements. \\
\hline $\begin{array}{l}\text { Initial Method } \\
\text { Detection Limit } \\
\text { Determination }\end{array}$ & $\begin{array}{l}\text { Multiple extraction and analysis of a low } \\
\text { concentration standard. Requirements were met. } \\
\text { (For } 2 \mathrm{~g} \text { sample the MDL for Aroclor } 1248=3.71 \\
\mathrm{ug} / \mathrm{kg} \text {, documented in files). }\end{array}$ \\
\hline Initial Calibration & $\begin{array}{l}\text { Five point Aroclor calibrations for } 1016,1248 \text {, } \\
1254 \text {, and } 1260 \text { were performed on } 5 / 21 / 98 \text { at } 20 \mathrm{ng} / \mathrm{mL} \\
\text { to } 400 \mathrm{ng} / \mathrm{mL} \text {, and three point calibrations were } \\
\text { performed for Aroclors } 1232 \text {, and } 1242 \text { at } 100 \mathrm{ng} / \mathrm{mL} \\
\text { to } 400 \mathrm{ng} / \mathrm{mL} \text {. The } 1221 \text { 5-point was calibrated on } \\
6 / 22 / 98 \text { at } 40-800 \mathrm{ng} / \mathrm{mL} \text {. }\end{array}$ \\
\hline $\begin{array}{l}\text { Carry-over } \\
\text { evaluation }\end{array}$ & $\begin{array}{l}\text { An instrument blank was run after the calibration } \\
\text { standards. No PCB carry-over was detected. Met } \\
\text { requirements. }\end{array}$ \\
\hline $\begin{array}{l}\text { Continuing } \\
\text { Calibration } \\
\text { Verifications (CCV) }\end{array}$ & $\begin{array}{l}\text { Aroclor } 1254 \text { was used to assess instrument perfor- } \\
\text { mance before and after the samples were analyzed on } \\
\text { it. The value found for the .200ng Aroclor } 1254 \\
\text { standard before the samples was. } 22248 \text { ng. This is } \\
111 \% \text { of the amount, and is within the } 15 \% \\
\text { (Deviation) acceptable. The other } 1254 \text { CCVs were } \\
107 \% \text {, } 112 \% \text {, 100\%, and } 114 \% \text {. The } 1221 \text { CCVs during } \\
\text { and after samples was } 104 \% \text {, and } 108 \% \text {, respectively. } \\
\text { CCVs were acceptable. }\end{array}$ \\
\hline $\begin{array}{l}\text { Laboratory Control } \\
\text { Sample }\end{array}$ & $\begin{array}{l}\text { A blank matrix spike blank was used as a Laboratory } \\
\text { Control Sample to assess method performance. This } \\
\text { contained Aroclor } 1254 \text {. Recovery was } 88 \% \text {, and met } \\
\text { requirements of } 70-130 \% \text {. }\end{array}$ \\
\hline
\end{tabular}




\section{HNF-SD-WM-DPP 309}

\section{RESULTS / DISCUSSION}

The Aroclors 1221 and 1254 were observed in the sample at high concentrations. such that a dilution was necessary to bring the amounts on column within the calibration curve. After careful comparison, it was noted that the Aroclors 1221, and 1254 were present in the samples. Aroclor 1221 is the predominant pattern. The amounts are reported per three peak quantitation in the attached resuit summary forms.

The PCBs were quantified in sample $\$ 98 B 000220$, on an as received, post centrifugation basis. The Aroclor $1221(25.4 \mathrm{mg} / \mathrm{Kg})$, and Aroclor $1254(8.43$ $\mathrm{mg} / \mathrm{Kg}$ ) were observed in the sample. The highest total of PCBs identified in the sample was $34 \mathrm{mg} / \mathrm{Kg}$. The duplicate and average result in Table IV is for reference. The peak at 10.8 minutes, which is Biphenyl, was not used for quantitation purposes, because it did not match the expected pattern ratio for 1221. There was a high bias noted for that non-PCB peak of Biphenyl observed, perhaps due to coelution. The peaks at 13.28, 13.67, and 13.83 minutes were used for quantitation of the 1221 present in the sample.

The Laboratory Control Spike (LCS) recovery was good. The Aroclor mixture of 1254 was used as an LCS, and was recovered at $88 \%$.

Surrogate spiking was performed on all samples using Tetrachloro-m-xylene (TCX) and Decachlorobiphenyl (DCB). and is summarized in Table II for the instrument blank, method blank, LCS, sample, duplicate, MS and MSD. According to U.S. Environmental Protection Agency advisory limits, set at $50-150 \%$, surrogate recoveries for these samples are acceptable. We are in the process of implementing surrogate control limits. Tentative control limits based on sample data that has been reported from this $1 \mathrm{ab}$ are $39-148 \%$ for TCX, and $43-$ $163 \%$ for DCB. Surrogate concentrations were determined by the 5-point (1254) calibration on 5-21-98.

Table II.

SURROGATE RECOVERIES (\%)

\begin{tabular}{|c|c|c|}
\hline Sample & TCX (\%) & DCB (\%) \\
\hline Instrument Blank & 92 & 100 \\
\hline Method Blank & 66 & 90 \\
\hline LCS & 70 & 97 \\
\hline S98B000220/100 & 93 & $194^{*}$ \\
\hline S98B0002200/100 & 107 & $175^{*}$ \\
\hline S98B000220MS $/ 300$ & 94 & $183^{*}$ \\
\hline S98B000220MSD $/ 300$ & 94 & $158^{*}$ \\
\hline
\end{tabular}

* surrogate outside surrogate advisory 7 imits of $50-150 \%$. 
HNF-SD-WM-DP-309 REV. 0

Table III.

SPIKE RECOVERIES (\%)

\begin{tabular}{|c|c|}
\hline Sample & Aroclor $1254(\%)$ \\
\hline S98B000220MS & 51.1 \\
\hline S98B000220MSD & 78.4 \\
\hline RPD & 42.2 \\
\hline
\end{tabular}

MS/MSD EPA advisory limits of $50-150 \%$

Table IV.

AROCLOR RESULTS

\begin{tabular}{|c|c|c|}
\hline AROCLOR & S98B000220 (ug/g) & S98B000220Dup (ug/g) \\
\hline TOTAL & 33.8 & 22.3 \\
\hline & AVG 28 & RPD 41 \\
\hline
\end{tabular}


HNF-SD-WM-DP-309 REV. 0

File:C: \wpdata\ross\PCBtkl00.gar

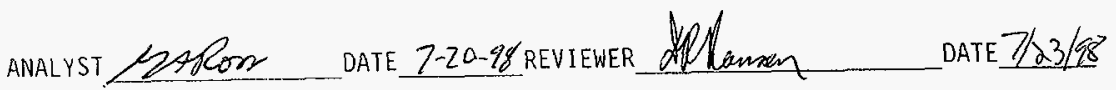




\section{Appendix $A$ \\ Comparison to $\mathrm{SW}-846$}

Method 3540C, Soxhlet Extraction, Revision 3, December 1996

\begin{tabular}{|c|c|c|c|}
\hline SW-846 States: & Procedure States: & ype of change. & $\begin{array}{l}\text { Reason for } \\
\text { Change? }\end{array}$ \\
\hline $\begin{array}{l}\text { Section } 7.2 \text { : Use } \\
5 \text { to } 10 \text { grams of } \\
\text { sample }\end{array}$ & $\begin{array}{l}\text { Step 9.5: Use } 1 \\
\text { to } 10 \text { grams of } \\
\text { sample }\end{array}$ & $\begin{array}{l}\text { Deviation- allows } \\
\text { scale down of the } \\
\text { sample and spike } \\
\text { additions } \\
\text { appropriately } \\
\text { (cut by } \\
\text { approximately } \\
1 / 4 \text { ) }\end{array}$ & $\begin{array}{l}\text { Due to } 1 \text { imited } \\
\text { amounts of } \\
\text { samples, waste } \\
\text { minimization and } \\
\text { ALARA concerns, } \\
\text { smaller amounts } \\
\text { of sample may } \\
\text { need to be used. }\end{array}$ \\
\hline $\begin{array}{l}\text { Section } 7.4 \text { : Use } \\
300 \mathrm{~mL} \text { solvent }\end{array}$ & $\begin{array}{l}\text { Step 9.5: Use } 90 \\
\mathrm{~mL} \text { solvent. }\end{array}$ & $\begin{array}{l}\text { Deviation-scale } \\
\text { down of the } \\
\text { sample, solvent, } \\
\text { and spike } \\
\text { additions } \\
\text { appropriately } \\
\text { (cut by } \\
\text { approximately } \\
1 / 4 \text { ) }\end{array}$ & $\begin{array}{l}\text { See above. This } \\
\text { al so reduces the } \\
\text { amount of methyl- } \\
\text { ene chloride } \\
\text { evaporated up the } \\
\text { hood. }\end{array}$ \\
\hline $\begin{array}{l}\text { Section } 7.4 \text { : Use } \\
500 \mathrm{~mL} \text { round } \\
\text { flask. }\end{array}$ & $\begin{array}{l}\text { Step 9.11: Use } \\
125 \mathrm{~mL} \text { round } \\
\text { flask with flat } \\
\text { bottom. }\end{array}$ & Deviation & $\begin{array}{l}\text { Smaller flasks } \\
\text { provide efficient } \\
\text { extraction in } \\
\text { limited hood } \\
\text { space. }\end{array}$ \\
\hline $\begin{array}{l}\text { Section } 7.10 \text { : } \\
\text { Add approximate } 7 y \\
50 \mathrm{~mL} \text { exchange } \\
\text { solvent once. }\end{array}$ & $\begin{array}{l}\text { Steps } 9.16 .1- \\
9.16 .2: \text { Add } 30 \\
\mathrm{~mL} \text { of hexane } \\
\text { three times }\end{array}$ & Deviation & $\begin{array}{l}\text { Repeating process } \\
\text { efficiently } \\
\text { removes first } \\
\text { solvent residue. }\end{array}$ \\
\hline $\begin{array}{l}\text { Section } 7.12 .2 \text { : } \\
\text { Place the } \\
\text { concentrator tube } \\
\text { in a warm bath } \\
\left(35{ }^{\circ} \mathrm{C}\right) \text { and } \\
\text { evaporate to } 0.5 \\
\text { mL... }\end{array}$ & $\begin{array}{l}9.25 .1 \text { : Place } \\
\text { the tube in the } \\
\text { Reacti-vap } \\
\text { evaporator } \\
\text { assembly, with } \\
\text { the heating } \\
\text { module preheated } \\
\text { to } 40^{\circ} \mathrm{C} . . \text {. } \\
\text { semivolatiles are } \\
\text { collected when } \\
\text { the Mecl level } \\
\text { reaches } 2 \mathrm{~mL} \text {. }\end{array}$ & Substitution & $\begin{array}{l}\text { Heat transfer is } \\
\text { done with a heat- } \\
\text { ing block instead } \\
\text { of a bath, } \\
\text { temperature } \\
\text { differences are } \\
\text { nominal. A final } \\
\text { volume of } 2 \mathrm{~mL} \\
\text { was necessary to } \\
\text { prevent losses. }\end{array}$ \\
\hline
\end{tabular}

\begin{tabular}{|c|c|c|}
\hline $\begin{array}{r}\text { Procedure No. } \\
\text { LA-523-138 }\end{array}$ & $\begin{array}{r}\text { Rev Mod } \\
\text { A-2 }\end{array}$ & $\begin{array}{r}\text { Page } \\
19 \text { of } 23 \\
\hline\end{array}$ \\
\hline
\end{tabular}




\section{Appendix D \\ Comparison to $\mathrm{SW}-846^{8}$}

Method 8082, Revision 0, December 1996, Polych7orinated Bipheny7s (PCBs) By Gas Chromatography

\begin{tabular}{|c|c|c|c|}
\hline SW-846 states: & Procedure States: & Type of Change: & Reason for Change: \\
\hline $\begin{array}{l}\text { Table } 3 \text { or step } 2.5 \text { : (GC } \\
\text { Operating Conditions or } \\
\text { summary) Injection } \\
\text { volume: } 2 \text { LL (with a } \\
0.54 \mathrm{~mm} \text { id. megabore } \\
\text { column). or step } 7.4 .5 \\
\text { A } 2-\mu L \text { injection of each } \\
\text { calibration standard is } \\
\text { recommended. Other } \\
\text { injection volumes may be } \\
\text { used if the analyst can } \\
\text { demonstrate adequate } \\
\text { sensitivity for the } \\
\text { compounds. } \\
\text {. }\end{array}$ & $\begin{array}{l}\text { Step 10.1: Gas } \\
\text { Chromatograph Operating } \\
\text { Conditions - Injection } \\
\text { Volume: } 1 \mu \mathrm{L}\end{array}$ & Substitution & $\begin{array}{l}\text { Smaller injection } \\
\text { needed for mid-bore } \\
\text { column }(0.32 \mathrm{~mm} \text { id). } \\
\text { otherwise injection of } \\
\text { oit sample extracts } \\
\text { will compromise the } \\
\text { system. The "Suggested" } \\
\text { calibration levels of } \\
100,150,200,400 \text {, and } \\
600 \text { ng/mL extends above } \\
\text { the linear calibration } \\
\text { range for a } 1 \mu \text {. } \\
\text { injection. Hence the } \\
\text { calibration levels } \\
\text { selected are at } 20,50 \text {, } \\
100,200 \text {, and } 400 \\
\text { ng/mL. }\end{array}$ \\
\hline $\begin{array}{l}\text { Step 5.5: Stock } \\
\text { standard solutions ( } 1000 \\
\mathrm{mg} / L)\end{array}$ & $\begin{array}{l}\text { Step } 4.1 .2: \text {... the oC } \\
\text { reference sample } \\
\text { concentrate should } \\
\text { contain the most repre- } \\
\text { sentative multicomponent } \\
\text { parameter at a concen- } \\
\text { tration of } 50 \mathrm{mg} / \mathrm{L} \text {. (In } \\
\text { fact, } 40 \mathrm{mg} / \mathrm{L} \text { standards } \\
\text { custom made by Restek } \\
\text { are used so that the } \\
\text { threshold limit of } 50 \\
\text { PPM will not be met or } \\
\text { exceeded.) }\end{array}$ & $\begin{array}{l}\text { Deviation- scale down of } \\
\text { the spike additions } \\
\text { appropriately (cut by } \\
\text { approximately } 1 / 100 \text { ). }\end{array}$ & $\begin{array}{l}\text { TSCA Regulation } \\
\text { concerns, waste } \\
\text { minimization, otherwise } \\
\text { all spiked matrices } \\
\text { must be isolated and } \\
\text { treated as mixed } \\
\text { regulated waste without } \\
\text { a disposal path. Also, } \\
\text { the level of QC spike } \\
\text { suggested is above the } \\
\text { calibration levels of } \\
\text { the GC. }\end{array}$ \\
\hline $\begin{array}{l}\text { Section } 8.3 .1: \text { A } 1 \mathrm{~mL} \\
\text { volume of the (QC) } \\
\text { concentrate spiked into } \\
1 \mathrm{~L} \text { of organic free } \\
\text { water will result in a } \\
\text { sample concentration of } \\
10-50 \mu \mathrm{g} / \mathrm{L} \text {. }\end{array}$ & $\begin{array}{l}\text { See step } 9.1 .5 .2 \text { of } \\
\text { LA-S23-115: If not, add } \\
\text { the water diluent first, } \\
\text { so the sample plus } \\
\text { diluent equals } 100 \mathrm{~mL} \text {. }\end{array}$ & Deviation & $\begin{array}{l}\text { Custom semi-micro } \\
\text { liquid- } 1 \text { iquid extractor } \\
\text { used in LA-523- } 115 \\
\text { requires less volume } \\
\text { (ca. } 100 \text { mL) ALARA } \\
\text { concerns and limited } \\
\text { sample and hood size } \\
\text { make smaller extraction } \\
\text { vessel a necessity. }\end{array}$ \\
\hline $\begin{array}{l}8081 \mathrm{~A} \text { and } 8082 \text { are } \\
\text { separate methods. }\end{array}$ & $\begin{array}{l}8081 A \text { and } 8082 \text { have been } \\
\text { combined. }\end{array}$ & Two methods combined. & $\begin{array}{l}\text { Sulfuric acid cleanup } \\
\text { limits pesticide } \\
\text { applicability. Acid } \\
\text { cleanup rarely used for } \\
\text { pesticides. }\end{array}$ \\
\hline
\end{tabular}

${ }^{8}$ NOTE: $\quad$ See LA-523-115 and LA-523-138 for sample preparation comparisons.

Procedure No.

LA-523-136
Rev Mod

A-1

39 of 39 
HNF-SD-WM-DP-309, REV. 0

ATTACHMENT 3

SAMPLE BREAKDOWN DIAGRAMS

15 
HNF-SD-WM-DP-309, REV. 0

THIS PAGE INTENTIONALLY LEFT BLANK 


\section{Tank 100 Sump Samples}

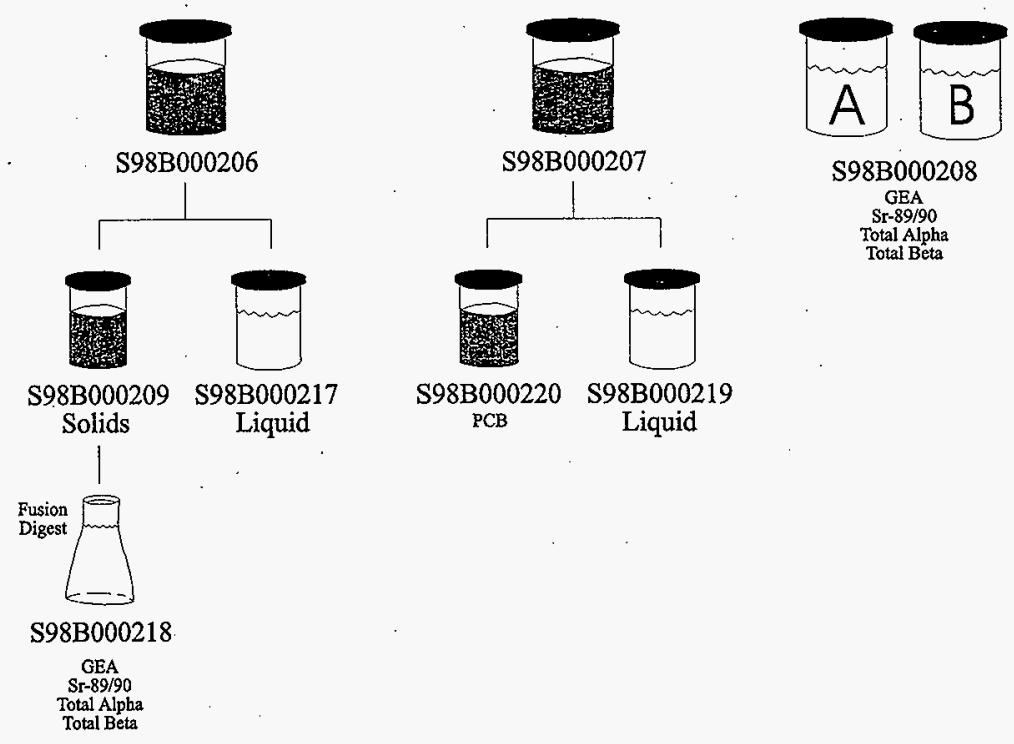


HNF-SD-WM-DP-309, REV. 0

ATTACHMENT 4

CHIAN OF CUSTODY AND REQUEST FOR SAMPLE ANALYSIS 
HNF-SD-WM-DP-309, REV. 0

THIS PAGE INTENTIONALLY LEFT BLANK

19 


\section{REQUEST FOR SAMPLE ANALYSIS (RSA)}

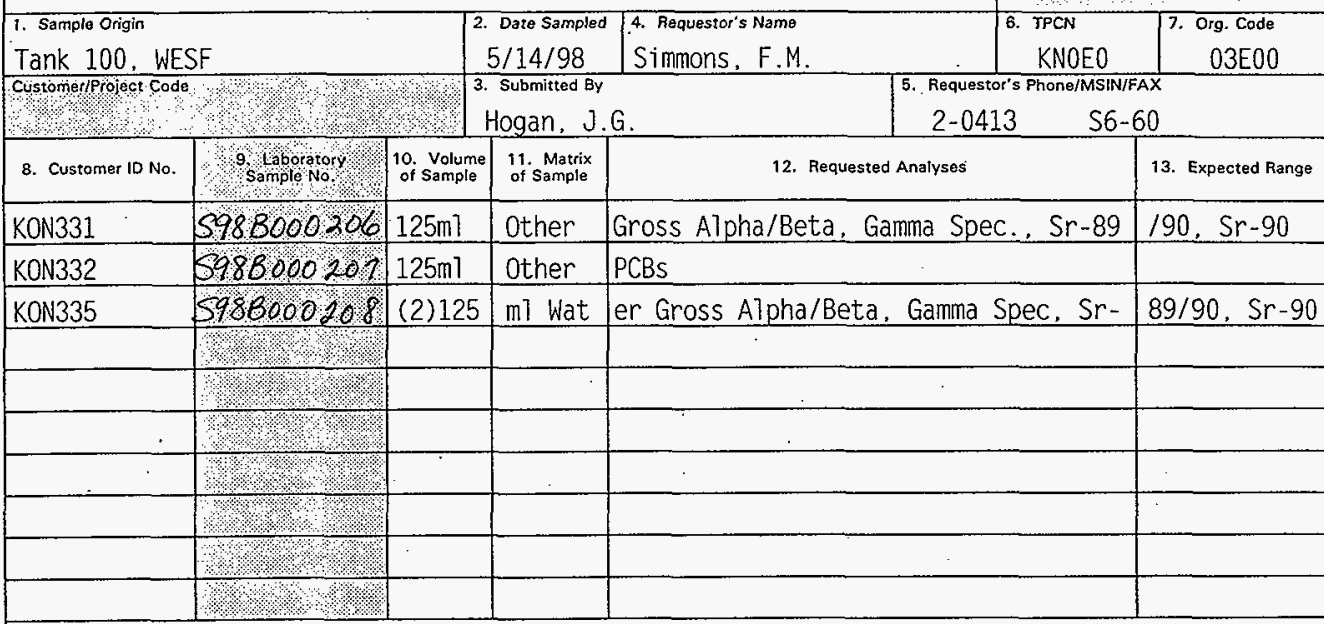

14. Does sample have a MSDS?

$\square$ Yes HEHF assigned MSOS No.

$[X]$ No Description of process that produced waste/sample:

Low Level Liquid Waste from WESF

15. Is this sample RCRA listed? $\square$ Yes $[X]$ No

Applicable Listed Waste Codes:

$\square$ Yes $\square$ No $\quad$ P Codes: (list)
$\square$ Yes $\square$ No $\quad$ U Codes: (list)
$\square$ Yes $\square$ No $\quad$ K Codes: (list)
$\square$ Yes $\square$ No $\quad$ F Codes: (list)

PCB: Does this waste/sample contain PCBs?
$\square$ Yes Over 500 ppm
If YES, what is the source of the PCBS?
पYes Over $50 \mathrm{ppm}$
$\square$ Transformer, capacitor, or ballast
[D] Yes PCBs are suspected
$\square$ Other, specify
$\square$ No PCBs are suspected
[X] Unknown

16. Sample Disposition

$\square$ Return to Customer

[X] Samples found to contain PCBs will be returned to the customer

$\square$ Dispose of per facility procedures with applied charges for analyses and disposal

Applicable Characteristic Codes:

$\begin{array}{ll}\square \text { Yes } \square \text { No } & \text { Do01: (how determined) } \\ \square \text { Yes } \square \text { No } & \text { D002: (how determined) } \\ \square \text { Yes } \square \text { No } & \text { Do03: (how determined) } \\ \square \text { Yes } \square \text { No } & \text { Toxic: (list codes) }\end{array}$

17. ac Required $\quad[]$ Per 222-S Laboratory Quality Assurance Plan (HNF-SD-CP,QAPP-016)

$\square$ Other (list reference document or attach)

18. Special Instructions (Special Storage Requirements, Reporting format, holding times, etc.)

S.A.F.\#S R98-060, R98-061, R98-063

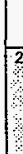

20 sample Regsuved $8 y$,

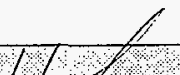

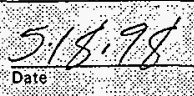

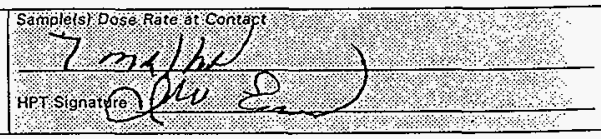

* 
HNF-SD-HM-DP-309, REV. 0

ATTACHMENT 5

222-S EXTRACTION BENCH SHEET

22 
HNF-SD-WM-DP-309, REV. 0 


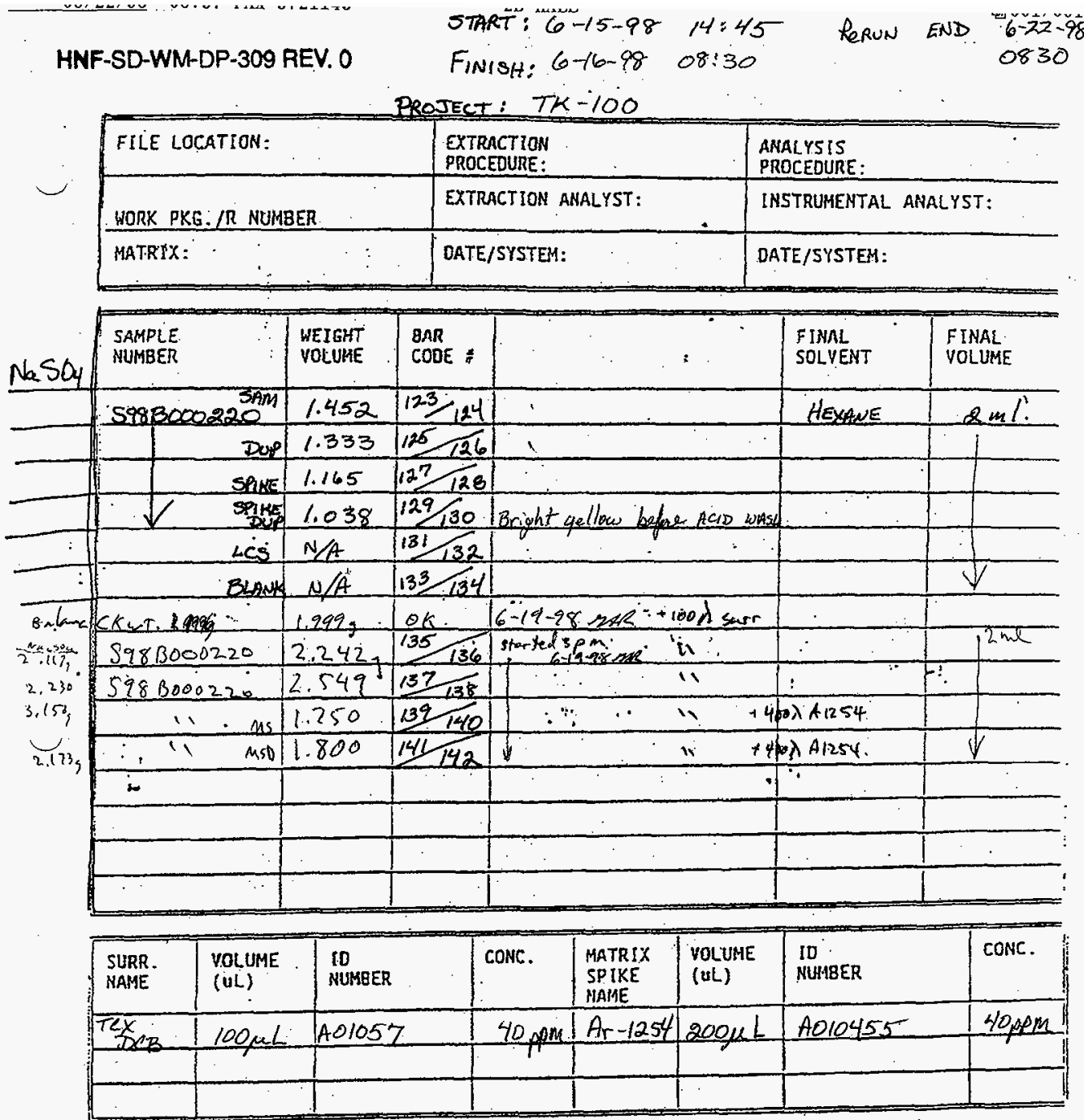

\begin{tabular}{|c|c|c|c|c|c|}
\hline $\begin{array}{l}\text { ANALYSIS } \\
\text { YOLUME } \\
\text { OF EXTRACT } \\
\text { USED }\end{array}$ & $\begin{array}{l}\text { INTERMAL } \\
\text { STANDARD } \\
\text { SUPPLIER }\end{array}$ & $\begin{array}{l}\text { VOLUME } \\
\text { ADOED } \\
\text { (UL) }\end{array}$ & $\begin{array}{l}\text { ID MUMBER } \\
\text { LOT NUHBER }\end{array}$ & $\begin{array}{l}\text { CONC. } \\
\text { IMITIAL }\end{array}$ & $\begin{array}{l}\text { COMC. } \\
\text { FIHAL }\end{array}$ \\
\hline & & $\cdot$ & & & \\
\hline
\end{tabular}


HNF-SD-WM-DP-309, REV. 0

ATTACHMENT 6

PCB RAW DATA 
HNF-SD-WM-DP-309, REV. 0

THIS PAGE INTENTIONALLY LEFT BLANK 
23 Jun $98 \quad 03: 50 \quad \mathrm{PM}$

Sequence: C: \HPCHEM $\backslash 2 \backslash S E Q U E N C E \backslash O 62298 A$.SEQ

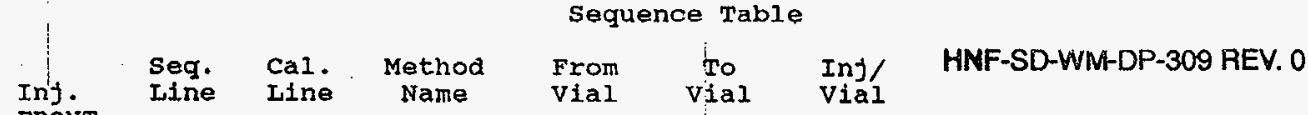

FRONT

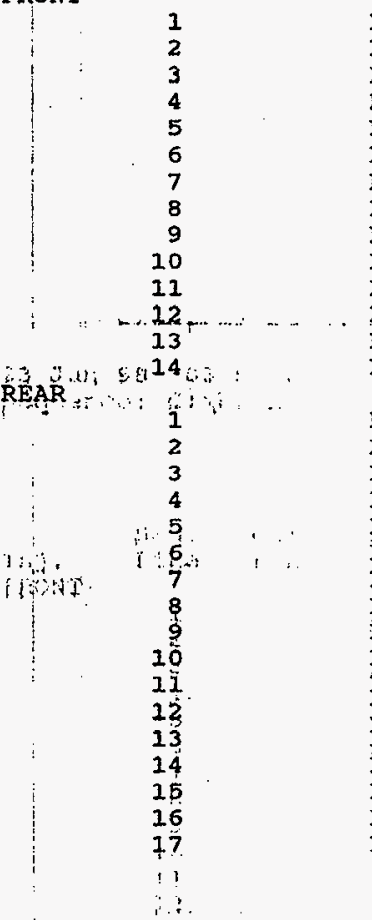

\begin{tabular}{|c|c|c|c|}
\hline PCB-FRNT & 2 & 1 & 3 \\
\hline PCB-FRNT & 2 & 2 & 2 \\
\hline PCB-FRNT & 3 & 12 & 1 \\
\hline PCB-FRNT & 12 & 16 & 1 \\
\hline PCB-FRNT & 13 & 13 & 1 \\
\hline PCB-FRNT & 17 & 27 & 1 \\
\hline PCB-FRNT & 13 & 23 & 1 \\
\hline PCB-FRNT & 18 & 18 & 1 \\
\hline PCB-FRNT & 13 & 13 & 1 \\
\hline PCB-FRNT & 19 & 19 & 1 \\
\hline PCB-FRNT & 13 & 13 & \\
\hline PCB-FRNT & 20 & 22 & 1 \\
\hline PCB-FRNT & 22 & 27 & 3 \\
\hline PCB - FRNT & 27 & 29 & \\
\hline PCB-REAR & 51 & 51 & 4 \\
\hline PCB-REAR & 52 & 52 & 2 \\
\hline PCB-REAR & 3 & 11 & 1 \\
\hline PCB-REAR & 53 & 53 & 2 \\
\hline PCB-REAR & 13 & a. 6 & 1 \\
\hline PCB-REAR & 13 & 13 & 2 \\
\hline PCB-REAR & 17 & 17 & $\mathbf{i}$ \\
\hline PCB-REAR & 13 & 13 & 1 \\
\hline PCB-REAR & 18 & 18 & $i$ \\
\hline PCB-REAR & 13 & 13 & 1 \\
\hline PCB-REAR & 19 & he & \\
\hline PCB-REAR & 13 & 13 & $i$ \\
\hline PCB-REAR & 20 & 51 & i \\
\hline PCB-REAR & 53 & 53 & 2 \\
\hline FCB-REAR & 23 & 26 & $\ddot{i}$ \\
\hline PCB-REAR & 54 & 54 & 1 \\
\hline PCB-REAR & 20 & 21 & $i$ \\
\hline$\vdots$ & sample & & 1 \\
\hline & & Taole & \\
\hline $\begin{array}{l}\text { Sample } \\
\text { Amount }\end{array}$ & & utiplier & $\begin{array}{l}\text { ISTD } \\
\text { Amount }\end{array}$ \\
\hline
\end{tabular}

$2000 \mathrm{ng} / \mathrm{ml}$ A1254, $400 \mathrm{ng} / \mathrm{ml}$ A.1254 $800 \mathrm{ng} / \mathrm{ml}$ A1221 $400 \mathrm{~g} / \mathrm{ml}$ A1221 $200 \mathrm{ng} / \mathrm{mI}$ A1221 $100 \mathrm{ng} / \mathrm{ml}$ A1221

Hong/mI A122I mid 7-22=-58

$200 \mathrm{ng} / \mathrm{ml}$ Al232

$200 \mathrm{ng} / \mathrm{ml} \mathrm{A1242}$

$200 \mathrm{ng} / \mathrm{ml}$ A1248

$200 \mathrm{ng} / \mathrm{ml}$ A 1660

$200 \mathrm{ng} / \mathrm{ml} \times 1254, \mathrm{civ}$

ibink

$131 \mathrm{LCS} / 20$

$133 \mathrm{mblnk} / 30,0+k 6-29-98$

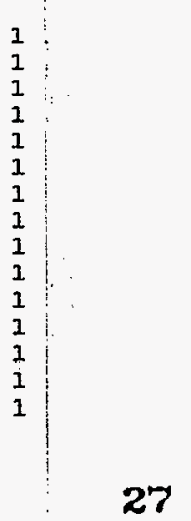


23 Jun 98 O3:50 PM

Sequence: $C$ : $\backslash H P C H E M \backslash 2 \backslash S E Q U E N C E \backslash 062298 A . S E R$

$\begin{array}{ll}\text { Vial Sample Name } & \text { Sample } \\ \text { Num. } & \text { Amount }\end{array}$

Sample Table

$16: 135$ tk100/300

17 137tk100/300

18. i39tk100/300Ms

19. 141tk100/300MSD

20 200rig/m1 A16605 mAR $720-18$

21 $400 \mathrm{ng} / \mathrm{ml}$ Al221

22 20ong/mi A1254 CLV

23: 135tk100/100

24 iblnk

25 $\quad 137 t k 100 / 100$

26 iblink .27 +. 20 कong/m1 A 1254 ccV

$\begin{array}{ll}29 . & 200 \mathrm{ng} / \mathrm{ml} \text { A } 1660 \\ 51: 1.2000 \mathrm{ng} / \mathrm{ml} \text { A1254 }\end{array}$

52 400ng/ml A1254

53 200ng/mi A1254

$54 \quad 200 \mathrm{ng} / \mathrm{mI}$ A1254

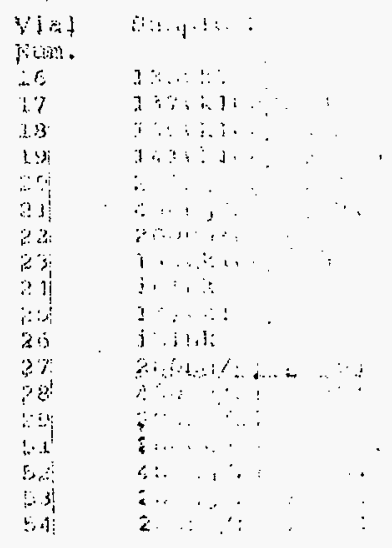

\section{t}

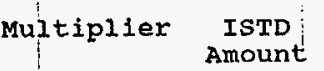

1
1
1
1
1
1
1
1
1
1
1
1
1
1
1
1
1
1

1
1
1
1
1
1
1
1
1
1
1
1
1
1
1
1

HNF-SD-WAH-DP-309 REV. 0

Amount 


\title{
INITIAL CALIBRATION DATA
}

\author{
Start Cal Date : 06-AUG-97 17:19 \\ End Cal Date : 22-JUN-98 19:59 \\ Quant Method : ESTD \\ Origin : Force \\ Target Version: 3.10 \\ Integrator : HP Genie \\ Method file : /chem/gc2.i/062298.b/rcrapcb.m \\ Cal Date : 24-Jun-1998 09:07 \\ Curve Type : Average
}

Calibration File Names:

Level 1:/chem/gc2.i/062298.b/007f0301.d

Level 2:/chem/gc2.i/062298.b/006f0301.d

Level 3:/chem/gc2.i/062298.b/005f0301.d

Level 4:/chem/gc2.i/062298.b/004f0301.d

Level 5: /chem/gc2.i/062298.b/003f0301.d

\begin{tabular}{|c|c|c|c|c|c|c|c|c|}
\hline & \multirow[b]{2}{*}{ Compound } & 0.0200 & 0.0500 & 0.1000 & 0.2000 & 0.4000 & & \\
\hline & & Level 1 & Level 2 & Level 3 & Level 4 & Level 5 & $\overline{R R F}$ & $\%$ RSD \\
\hline \multicolumn{9}{|c|}{ 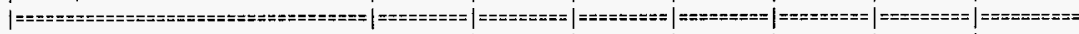 } \\
\hline 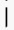 & 1 alpha-BHC & +++++ & ++++ & ++++ & ++++ & +++++ & +++++ & ++++ \\
\hline 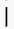 & 2 beta- 8 HC & +t+++ & ++++ & ++++ & +++++ & +++++ & ++++ & ++++ \\
\hline 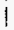 & 3 delta-BHC & +++++ & +++++ & +++++ & +++++ & +++++ & +++++ & +++++ \\
\hline & 4 gamma-BHC ( $L$ indane) & +++++ & +++++ & +++++ & +++++ & +++++ & +++++ & +++++ \\
\hline f & 5 Heptachlor & ++++ & +++++ & +++++ & +++++ & +++++ & +++++ & +++++ \\
\hline 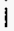 & 6 Aldrin & ++++1 & +++++ & +++++ & +++++ & +++++ & +++++ & +++++ \\
\hline & 7 Heptachlor epoxide & $++++\infty$ & ++++++ & +++++ & +++++ & ++++ & +++++ & +++++ \\
\hline 1 & 8 Endosulfan 1 & +++++ & +++++ & +++++ & +++++ & +++++ & +++++ & ++++ \\
\hline | & 9 Dieldrin & +++++ & +++++ & ++++ & +++++ & +++++ & +++++ & +++++ \\
\hline i & $104,4^{\prime}-D D E$ & ++++ & +++++ & +++++ & ++++ & +++++ & $t+t+t$ & $t+t+t$ \\
\hline 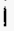 & 11 Endrin & +++++ & +++++ & +++++ & +++++ & $++++t$ & +++++ & +++++ \\
\hline & 12 Endosulfan II & +++++ & ++++++ & +++++ & +++++ & ++++ & +++++ & +++++ \\
\hline 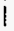 & $134,4^{\prime}-000$ & +++++ & +++++ & +++++ & +++++ & $+4+++$ & +++++ & +++++ \\
\hline 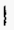 & 14 Endosulfan sulfate & $++t+t$ & +++++ & +++++ & +++++ & $t++++$ & ++++ & +++++ \\
\hline I & $154,4^{\prime}=00 \mathrm{~T}$ & +++++ & +++++ & ++++ & ++++ & +++++ & ++++ & +++++ \\
\hline 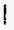 & 16 Merhoxychlor & +++++ & +++++ & +++++ & +++++ & $4++++$ & +++++ & +++++ \\
\hline 1 & 17 Endrin ketone & +++++ & +++++ & +++++ & +++++ & +++++ & +++++ & +++++ \\
\hline 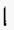 & 18 Endrin aldehyde & +++++ & +++++ & +++++ & ++++ & +++++ & +++++ & $++t++$ \\
\hline 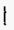 & 19 alpha-Chlordane & $++++t$ & +++++ & +++++ & ++++ & +++++ & ++++ & +++++ \\
\hline & 20 gamma-Chlordane & +++++ & +++++ & +++++ & $+++t+$ & $++++t$ & ++++ & ++++ \\
\hline & 21 Toxaphene(1) & +++++ & +++++ & +++++ & +++++ & +++++ & +++++ & $++++t$ \\
\hline 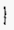 & (2) & +++++ & +++++ & ++++ & +++++ & +++++ & +++++ & +++++ \\
\hline & (3) & $++++\quad \mid$ & $++++\quad \mid$ & +++++ & +++++ & $++++\quad \mid$ & +++++ & +++++ \\
\hline & 22 Aroctor-1016(1) & 685050 & 706900 & 706720 & 622025 & 513460 & 646831 & 12.716 \\
\hline & (2) & 798050 & 774260 & 801400 & 717850 & 592898 & 736892 & 11.830 \\
\hline & (3) & 987100 & 981500 & 1026050 & 940650 & 784878 & 944036 & 9.955 \\
\hline & 23 Aroctor $-1221(1)$ & 167720 & 1940701 & 234670 & 214092 & 178668 & 197847 & 13.630 \\
\hline 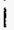 & (2) & $62160 \mid$ & 77090 & 76970 & 84552 & 72731 & 74701 & 10.979 \\
\hline & (3) & 180975 & 164390 & 220580 & 217148 & 200075 & 196637 & 12.169 \\
\hline & (4) & 235075 & 231000 & 230465 & 223318 & 208110 & 225595 & 4.722 \\
\hline & (5) & 478100 & 470360 & 563445 & 524620 & 458450 & 498999 & 8.798 \\
\hline
\end{tabular}


222-S Laboratory

\section{INITIAL CALIBRATION DATA}

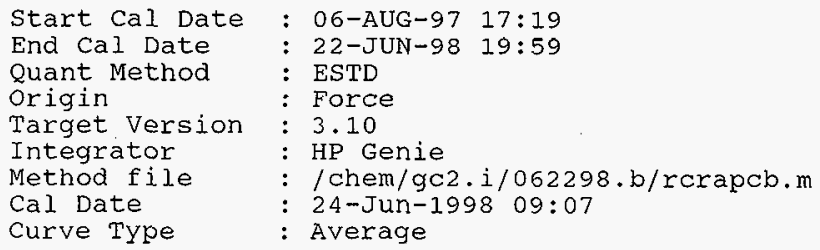

\begin{tabular}{|c|c|c|c|c|c|c|c|c|}
\hline & & 0.0200 & 0.0500 & 0.1000 & 0.2000 & 0.4000 & & 1 \\
\hline & Compound & | Level 1 | & Level 2 I & | Level 3 | & Level 4 & | Level 5 | & RRF & $\%$ RSD \\
\hline & 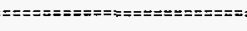 & $=\mid=== \pm= \pm== \pm 1$ & $===\varepsilon====-i$ & l===="===-= & $== \pm=n= \pm==\mid$ & 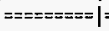 & $==\geq=====* \mid$ & $== \pm=ニ====0$ \\
\hline & 24 Aroctor $-1232(1)$ & $1++++$ & +++++ & 549340 & 559235 & 460210 & 522928 & 10.430 \\
\hline & (2) & $t+++t$ & ++++ & 326870 & 350830 & $292162 \mid$ & 323288 & 9.124 \\
\hline & (3) & $+t+t$ & +++++ & 4017301 & 437850 & $394132 \mid$ & 411238 & 5.680 \\
\hline & 25 Aroclor $-1242(1)$ & ++++ & +++++ & 4675600 & 512610 & 456095 & 478755 & 6.2401 \\
\hline & $(2)$ & ++++ & +++++ & 509520 & 576045 & 530280 & 538615 & 6.319 \\
\hline & (3) & $t++t+\quad \mid$ & +++++ & 625990 & 731335 & 693558 & 683628 & $7.807 \mid$ \\
\hline & 26 Aroclor $-1248(1)$ & 767300 & 692800 & 6778301 & 720975 & 629835 & 697748 & 7.311 \\
\hline & (2) & 675800 & 635180 & 628660 & 688730 & 616158 & 648906 & 4.861 \\
\hline & (3) & $910550 \mid$ & 840920 & $815560 \mid$ & 8992151 & 803228 & 853894 & 5.698 \\
\hline & 27 Aroclor $-1254(1)$ & 827300 & 812780 & 803440 & 839655 & 792698 & 815174 & 2.291 \\
\hline & $(2)$ & 1101500 & 1086100 & 1060040 & 1086780 & 1057750 & 1078434 & 1.7511 \\
\hline & (3) & 936000 & 941120 & 936560 & 1003545 & 1020202 & 967486 & 4.237 \\
\hline & 28 Aroclor $-1260(1)$ & 1056950 & 1017260 & 10406301 & 979115 & 775998 & 973990 & 11.754 \\
\hline & $(2)$ & 1060000 & 994400 & 1034930 & 982735 & $769212 \mid$ & 968256 & 11.930 \\
\hline & $(3)$ & $|860200|$ & 901620 & 9609801 & 9574001 & $753830 \mid$ & $886806 \mid$ & 9.617 \\
\hline \multicolumn{9}{|c|}{ 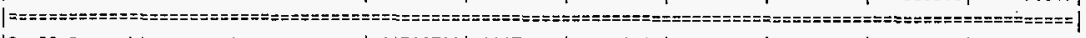 } \\
\hline$\$$ & 29 Tetrachloro-m-xylene & $|14389700|$ & $14672520 \mid$ & 16410640 이 & 142548601 & 13998965 & $14745337 \mid$ & 6.525 \\
\hline$\$$ & 30 decachl orobiphenyt & | $8633700 \mid$ & 8030800 & 8248180 & 7121710 & 8643725 & 8135503 & 7.6701 \\
\hline
\end{tabular}




\section{HNF-SD-WM-DP-309 REV. 0}

\section{2-S Laboratory}

Data file : /chem/gc2.i/062298.b/002f0202.d

Lab Smp Id: $400 \mathrm{ng} / \mathrm{ml}$ A 1254

Inj Date : 22-JUN-98 15:09

Operator : Gerald Ross

Smp Info : 400ng/ml A1254

Misc Info : $400 \mathrm{ng} / \mathrm{ml} \mathrm{A} 1254$

comment :

Method : /chem/gc2.i/062298.b/rcrapcb.m

Meth Date : 24-Jun-1998 09:58 gar

Cal Date : 22-JUN-98 19:59

Als bottle: 1

Dil Factor: 1.000

Integrator: HP Genie

Target Version: 3.10
Client Smp ID: $400 \mathrm{ng} / \mathrm{mI}$ A1254

Inst ID: $g c 2 . i$

Quant Type: ESTD

Cal File: 007f0301.d

compound sublist: AR1254.sub Sample Matrix: WATER

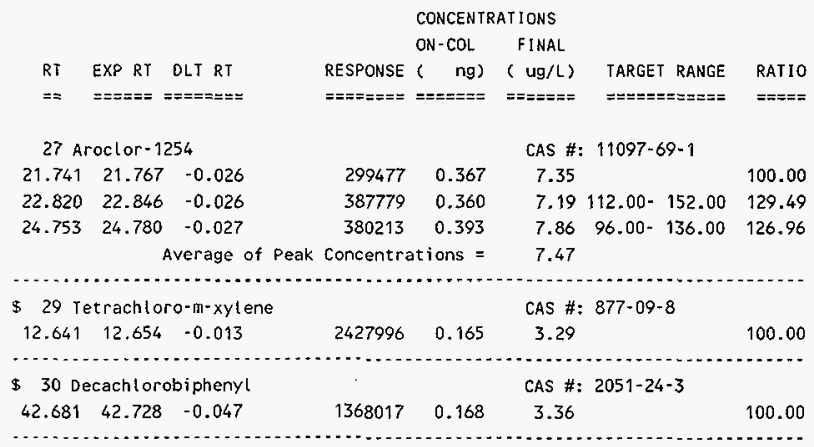


Data File: /chem/gc2.i/062298.b/002f0202.d

Report Date: 29-Jun-1998 13:37

$$
\begin{aligned}
& \text { 222-S Iaboratory } \\
& \text { RECOVERY REPORT }
\end{aligned}
$$

Client Name:

Sample Matrix: LIQUID

Client SDG: 062298

Fraction: PEST

Lab Smp Id: 400ng/ml A1254

Client Smp ID: $400 \mathrm{ng} / \mathrm{ml}$ A1254

Leve 1: Low

Data Type: GC MULTI COMP

Spikelist File: RESOLUTION.spk

Method File: / chem/gc2.i/062298.b/rcrapcb.m

\begin{tabular}{|c|c|c|c|c|}
\hline SURROGATE COMPOUND & $\begin{array}{l}\text { CONC } \\
\text { ADDED } \\
\text { UQ/L }\end{array}$ & $\begin{array}{c}\text { CONC } \\
\text { RECOVERED } \\
\text { UG } / \mathrm{L}\end{array}$ & $\begin{array}{c}\% \\
\text { RECOVERED }\end{array}$ & LIMITS \\
\hline $\begin{array}{lll}\$ & 29 & \text { Tetrachloro-m-xyle } \\
\$ & 30 & \text { Decachlorobiphenyl }\end{array}$ & $\begin{array}{l}4.00 \\
4.00\end{array}$ & $\begin{array}{l}3.29 \\
3.36\end{array}$ & $\begin{array}{l}82.33 \\
84.08\end{array}$ & $\begin{array}{l}50-150 \\
50-150\end{array}$ \\
\hline
\end{tabular}

Misc Info: $400 \mathrm{ng} / \mathrm{ml}$ A 1254 
Data File: /chem/gc2.i/062298.b/002f0202.d

Report Date: 29-Jun-1998 13:37

\section{2-S Laboratory \\ TARGET COMPOUNDS}

Client Name:

Lab Smp Id: 400ng/ml A1254

Sample Location:

Sample Date:

Sample Matrix: WATER

Analysis Type: PEST

Data Type: GC MULTI COMP

Misc Info: 400ng/ml A1254
Client SDG: 062298

Client Smp ID: $400 \mathrm{ng} / \mathrm{ml}$ A1254

Sample Point:

Date Received:

quant Type: ESTD

Level: LoW

Operator: Gerald Ross

CONCENTRATION UNITS:

( $\mathrm{ug} / \mathrm{L}$ or $\mathrm{ug} / \mathrm{KG}$ ) ug/L

\begin{tabular}{|c|c|c|}
\hline 11097-69-1------Aroclor -1254 & 7.47 & \\
\hline 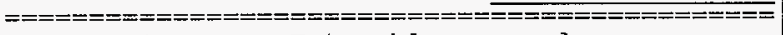 & $========-=$ & $====$ \\
\hline 877-09-8------Tetrachloro-m-xylene & 3.29 & \\
\hline $2051-24-3-------$ Decacn1oroblpnenyl & & \\
\hline
\end{tabular}




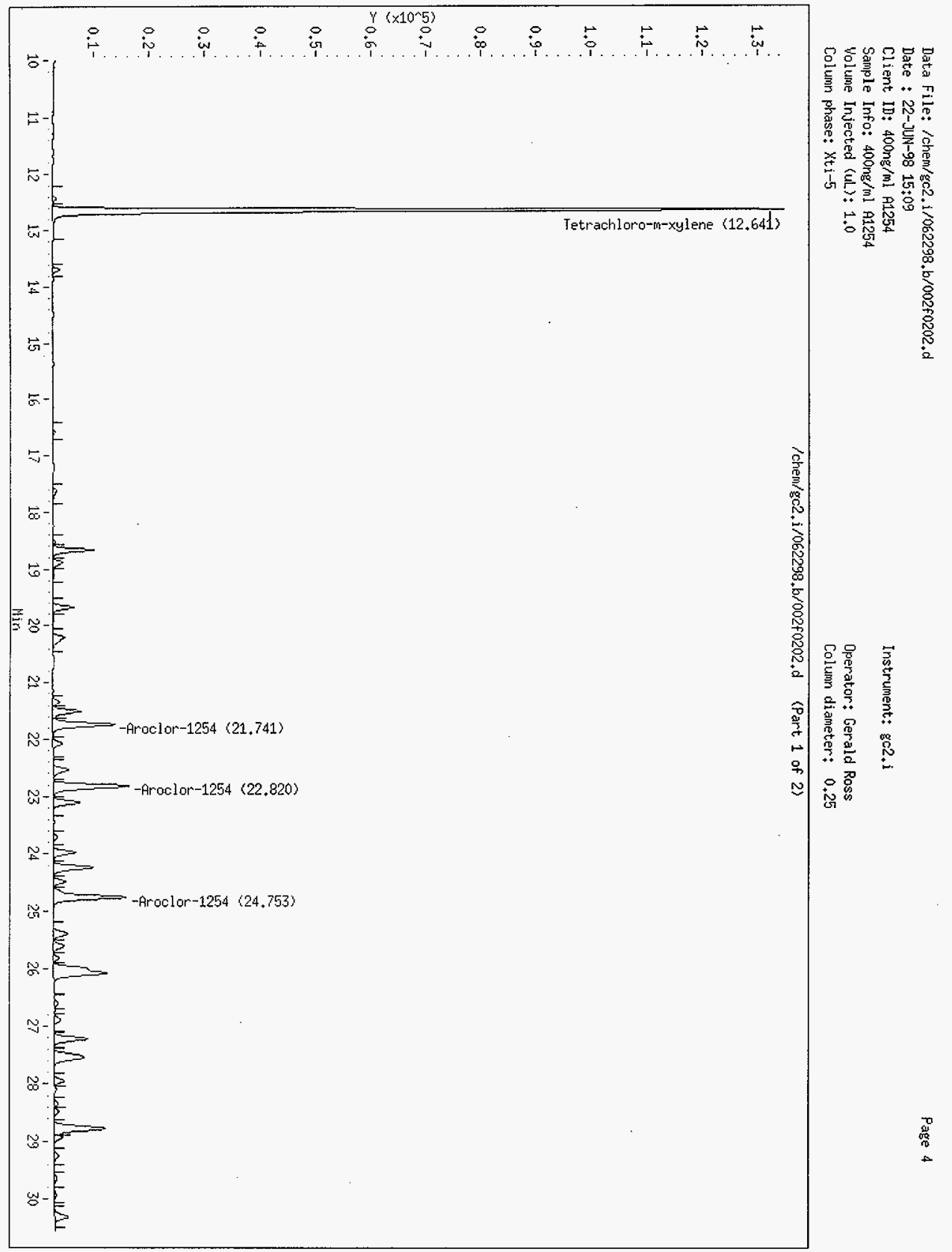


Date : 22-JUN-98 15:09

Instrument: $g \circ 2+i$

, 400ng/ml 11254

Sample Info: 400ing/ml A1254

Volume Injected $(\mathrm{uL}): 1.0$

Operator: Gerald Ross

Colurn phase: $x t i-5$

Column di ameter: 0.25

/chem/ $/ \mathrm{c} 2, \mathrm{i} / 062298, \mathrm{~b} / 002 \mathrm{f0} 0202, \mathrm{~d}$ (Part 2 of 2 )

1.3-

$1.2-$

$1.1-$

$1.0-$

$0.9-$

$0.8-$

$\hat{6}^{0.7-}$

$\tilde{\omega}$

$>0.6-$

$0.5-$

$0.4-$

$0.3-$

$0.2-$

$0.1-$

Al labaladel 


\title{
HNF-SD-WM-DP-309 REY. 0
}

\author{
222-S Laboratory
}

Data file: /chem/gc2,i/062298.b/003f0301.d

Lab Smp Id: $800 \mathrm{ng} / \mathrm{ml}$ A1221

Inj Date : 22-JUN-98 16:07

operator : Gerald Ross

Smp Info : $800 \mathrm{ng} / \mathrm{ml} \mathrm{A1221}$

Misc Info : $800 \mathrm{ng} / \mathrm{ml} \mathrm{A1221}$

Comment

Method : /chem/gc2.i/062298.b/rcrapcb.m
Meth Date : 24-Jun-1998 09:58 gar

Cal Date : 22-JUN-98 16:07

Als bottle: 1

Dil Factor: 1.000

Integrator: HP Genie

Target Version: 3.10
Client Smp ID: $800 \mathrm{ng} / \mathrm{ml}$ A1221

Inst ID: $g \mathrm{C} \cdot \mathrm{i}$

Quant Type: ESTD

Cal File: 003f0301.d

Calibration Sample, Level: 5

Compound Sublist: AR1221.sub

Sample Matrix: WATER

\begin{tabular}{|c|c|c|c|c|c|c|c|}
\hline & & & & AMOUN & & & \\
\hline & & & & CAL-AMT & $\mathrm{ON}-\mathrm{COL}$ & & \\
\hline$R T$ & EXP RT & DLT RT & RESPONSE & ( $\mathrm{ng})$ & ( $n g$ ) & TARGET RANGE & RATIO \\
\hline$==$ & $======$ & $=$ ====== & $====\pi===$ & $== \pm== \pm=$ & $======$ & 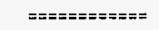 & $== \pm= \pm$ \\
\hline $23 \mathrm{Ar}$ & roctor-1 & 1221 & & & CAS \#: & : $11104-28-2$ & \\
\hline 10.801 & 10.811 & -0.010 & 142934 & & & & 100.00 \\
\hline 11.897 & 11.923 & -0.026 & 58185 & & & $75.00-115.00$ & 40.71 \\
\hline 13.276 & 13.282 & -0.006 & 160060 & 0.800 & 0.814 & $208.00-258.00$ & 111.98 \\
\hline 13.627 & 13.673 & .0 .046 & 166488 & 0.800 & 0.738 & $0.00-\quad 0.00$ & 116.48 \\
\hline 13.835 & 13.849 & -0.014 & 366760 & 0.800 & 0.735 & $0.00-$ & 256.59 \\
\hline & & Average of Peak & Amounts $=$ & & 0.749 & & \\
\hline$\$ 29 \mathrm{TC}$ & etrachlo & ro-m-xylene & & & CAS \#: & : $877-09-8$ & \\
\hline 12.640 & 12.654 & -0.014 & 4262207 & 0.200 & 0.289 & & 100.00 \\
\hline$\$ 30 \mathrm{De}$ & ecachlor & obipheny! & & & CAS \#: & $: 2051-24-3$ & \\
\hline 42.682 & 42.728 & -0.046 & 1845243 & 0.200 & 0.227 & & 100.00 \\
\hline
\end{tabular}


Data File: /chem/gc2.i/062298.b/003f0301.d

Report Date: 29-Jun-1998 13:37

$$
\begin{aligned}
& \text { 222-S Laboratory } \\
& \text { TARGET COMPOUNDS }
\end{aligned}
$$

Client Name:

Lab Smp Id: 800ng/ml A1221

Sample Location:

Sample Date:

Sample Matrix: WATER

Analysis Type: PEST

Data Type: GC MULTI COMP

Misc Info: $800 \mathrm{ng} / \mathrm{ml}$ A1221
Client SDG: 062298

Client Smp ID: $800 \mathrm{ng} / \mathrm{ml}$ A1221

Sample Point:

Date Received:

Quant Type: ESTD

Level: LOW

Operator: Gerald Ross
CAS NO.

COMPOUND
CONCENTRATION UNITS:

(ug/L or $u g / K G$ ) ug/L

\begin{tabular}{|c|c|c|}
\hline $\begin{array}{rl}11104-28-2-\cdots---A r o c l o r & 1221 \\
= & \end{array}$ & $========$ & $===$ \\
\hline 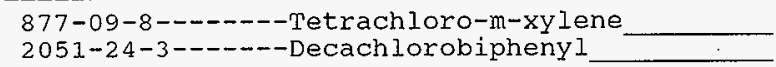 & $\begin{array}{r}5.78 \\
4.54\end{array}$ & \\
\hline
\end{tabular}


Date : 22-JUM-98 16:07

Instrument: gc2, i

Ong/ml A1221

Operator: Gerald Ross

Sample Info: 800ng/ml A122

Volume Injected $(u L): 1,0$

Column dianeter: 0,25

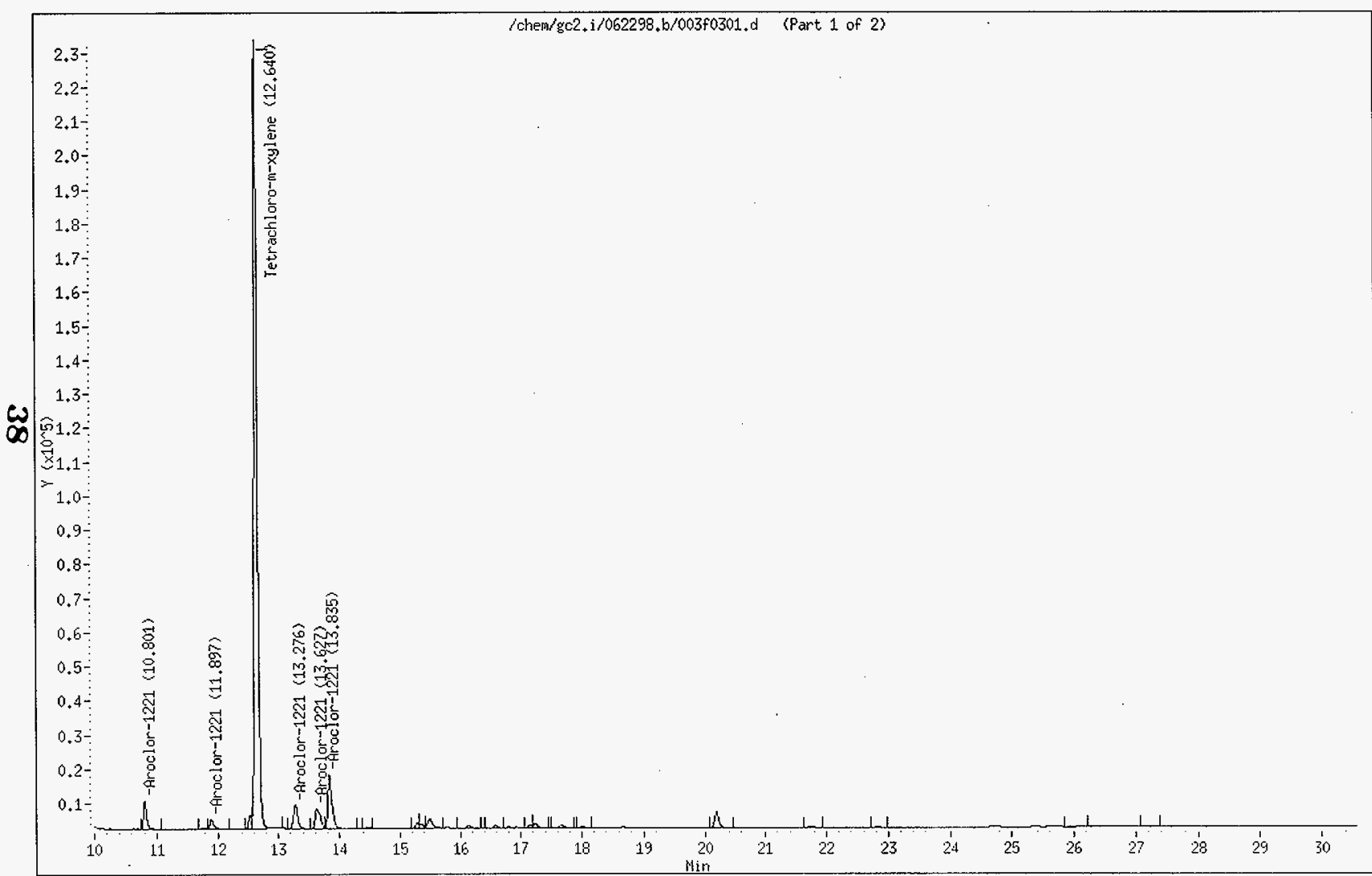


Data File: /chem/gc2, i/062298.b/003f0301.d

Date : 22-JUN-98 16:07

Client ID: $800 \mathrm{ng} / \mathrm{ml}$ A1221

Sarnple Info: 800ing/ml A1221

Volume Injected (uL): 1,0

Column phase: $\mathrm{Xti}_{\mathrm{t}}-\mathrm{5}$

Operator: Gerald Ross

Column diameter: 0,25

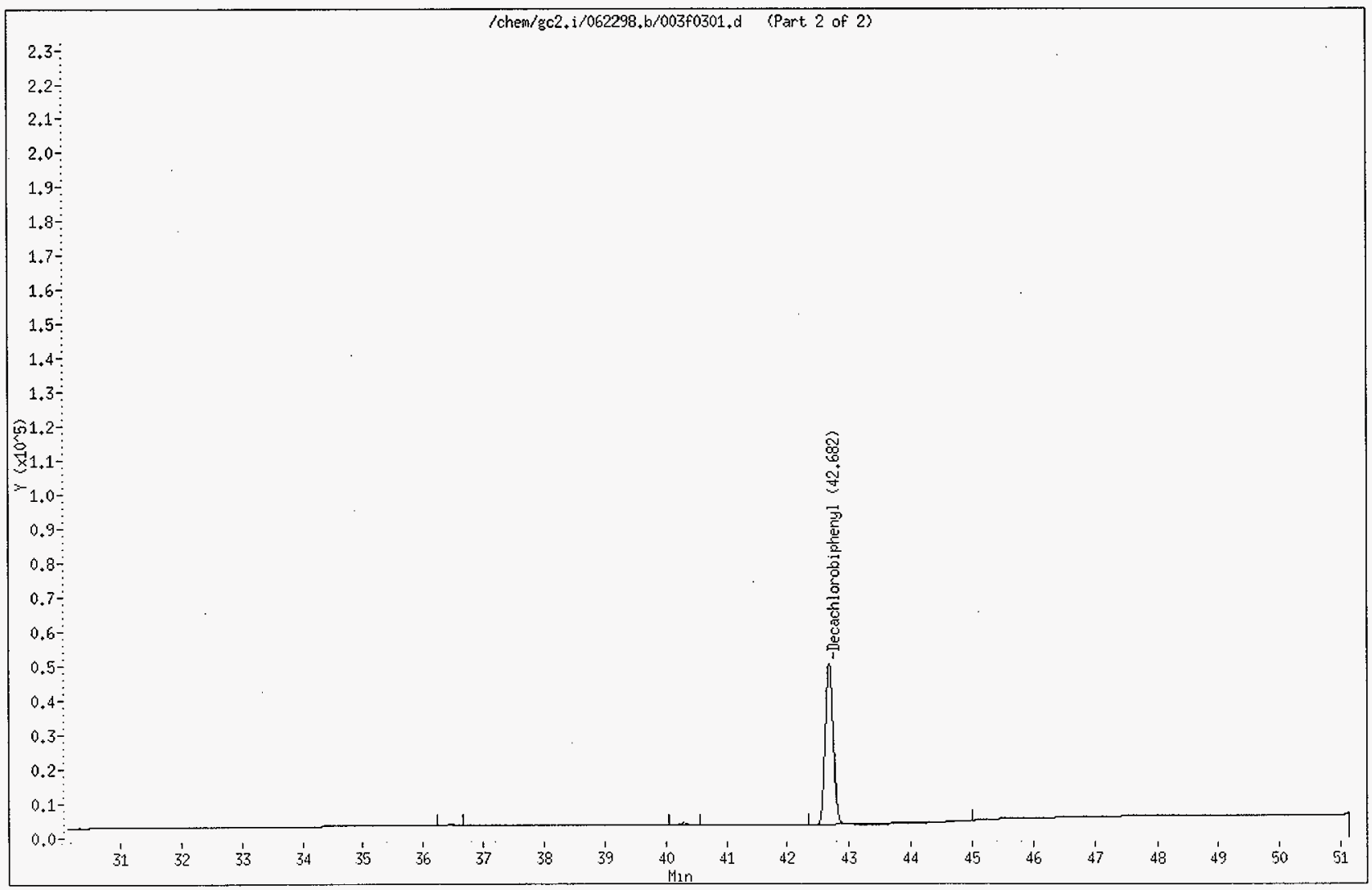


Data File: /chem/gc2.i/062298.b/004f0301.d Report Date: 29-Jun-1998 13:37

\section{2-S Laboratory}

Data file:/chem/gc2.i/062298.b/004f0301.d

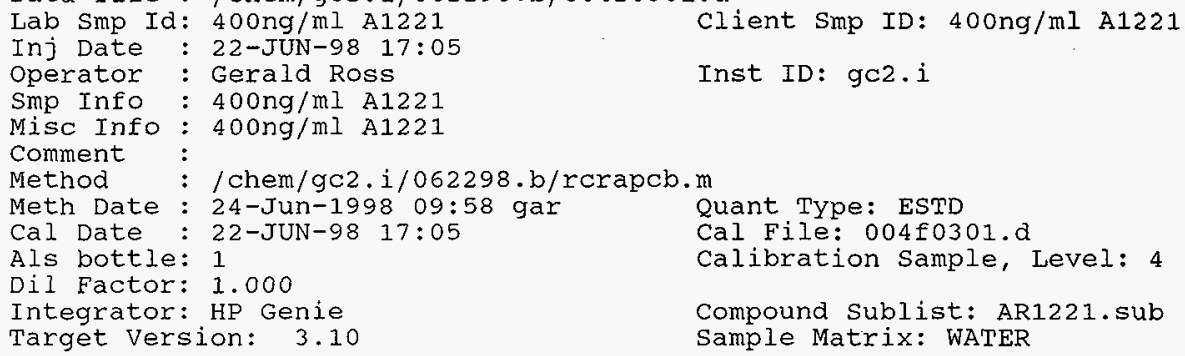

\begin{tabular}{|c|c|c|c|c|c|c|c|}
\hline & & & & AMOUN & & & \\
\hline & & & & CAL-AMT & $\mathrm{ON}-\mathrm{COL}$ & & \\
\hline RT & EXP RT & OLT RT & RESPONSE & ( ng) & ( ng) & TARGET RANGE & RATIO \\
\hline$==$ & $== \pm=\pi=$ & $\Rightarrow=\approx====0$ & $====\pi== \pm$ & $===:===$ & $==\Xi== \pm=$ & 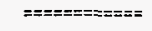 & $== \pm==$ \\
\hline $23 \mathrm{Ar}$ & roclor-12 & 1221 & & & CAS \#: & $11104-28-2$ & \\
\hline 10.804 & 10.811 & -0.007 & 85637 & & & & 100.00 \\
\hline 11.901 & 11.923 & -0.022 & 33821 & & & $75.00-115.00$ & 39.49 \\
\hline 13.279 & 13.282 & -0.003 & 86859 & 0.400 & 0.442 & $208.00-258.00$ & 101.43 \\
\hline 13.630 & 13.673 & -0.043 & 89327 & 0.400 & 0.396 & $0.00-\quad 0.00$ & 104.31 \\
\hline 13.837 & 13.849 & -0.012 & 209848 & 0.400 & 0.420 & $0.00-$ & 245.04 \\
\hline & & Average of Peak & Amounts $=$ & & 0.424 & & \\
\hline$\$ 29 \mathrm{Te}$ & trachtor & ro-m-xylene & & & CAS \#: & $877-09-8$ & \\
\hline 12.641 & 12.654 & -0.013 & 2510549 & 0.100 & 0.170 & & 100.00 \\
\hline$\$ 30 \mathrm{De}$ & ecachloro & obipheny: & & & CAS \#: & $2051-24-3$ & \\
\hline 42.681 & 42.728 & -0.047 & 1260662 & 0.100 & 0.155 & & 100.00 \\
\hline
\end{tabular}


Data File: /chem/gc2.i/062298.b/004f0301.d

Report Date: 29-Jun-1998 13:37

$$
\begin{aligned}
& \text { 222-S Laboratory } \\
& \text { TARGET COMPOUNDS }
\end{aligned}
$$

Client Name:

Lab Smp Id: 400ng/ml A1221

Sample Location:

Sample Date:

Sample Matrix: WATER

Analysis Type: PEST

Data Type: GC MULTI COMP

Misc Info: $400 \mathrm{ng} / \mathrm{ml}$ Al221
Client SDG: 062298

Client Smp ID: $400 \mathrm{ng} / \mathrm{ml}$ A1221

Sample point:

Date Received:

Quant Type: ESTD

Level: LOW

Operator: Gerald Ross

CONCENTRATION UNITS:

CAS NO.

COMPOUND

\begin{tabular}{|c|c|c|}
\hline $11104-28-2------$ Aroclor -1221 & 8.48 & \\
\hline 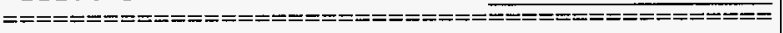 & $=======1==$ & $=====$ \\
\hline $\begin{array}{l}877-09-8-\cdots-1 \\
2051-24-3-\cdots---1 \text { - Decachlorobiphenyl }\end{array}$ & $\begin{array}{r}3.40 \\
3.10\end{array}$ & \\
\hline
\end{tabular}

(ug/I or $\mathrm{Ug} / \mathrm{KG}$ ) ug/L 
Data File: /chem/gc2, i/o62298,b/004f0301,d

Date : 22-JuN-98 17:05

Client ID: 400ng/ml A1221

Sample Info: 400ng/ml A1221

Volume Injected (uL) $: 1.0$

Instrument $¥ g c 2$.

Columin phase: Kti-5

Operator: Gerald Ross

Column diameter: 0.25

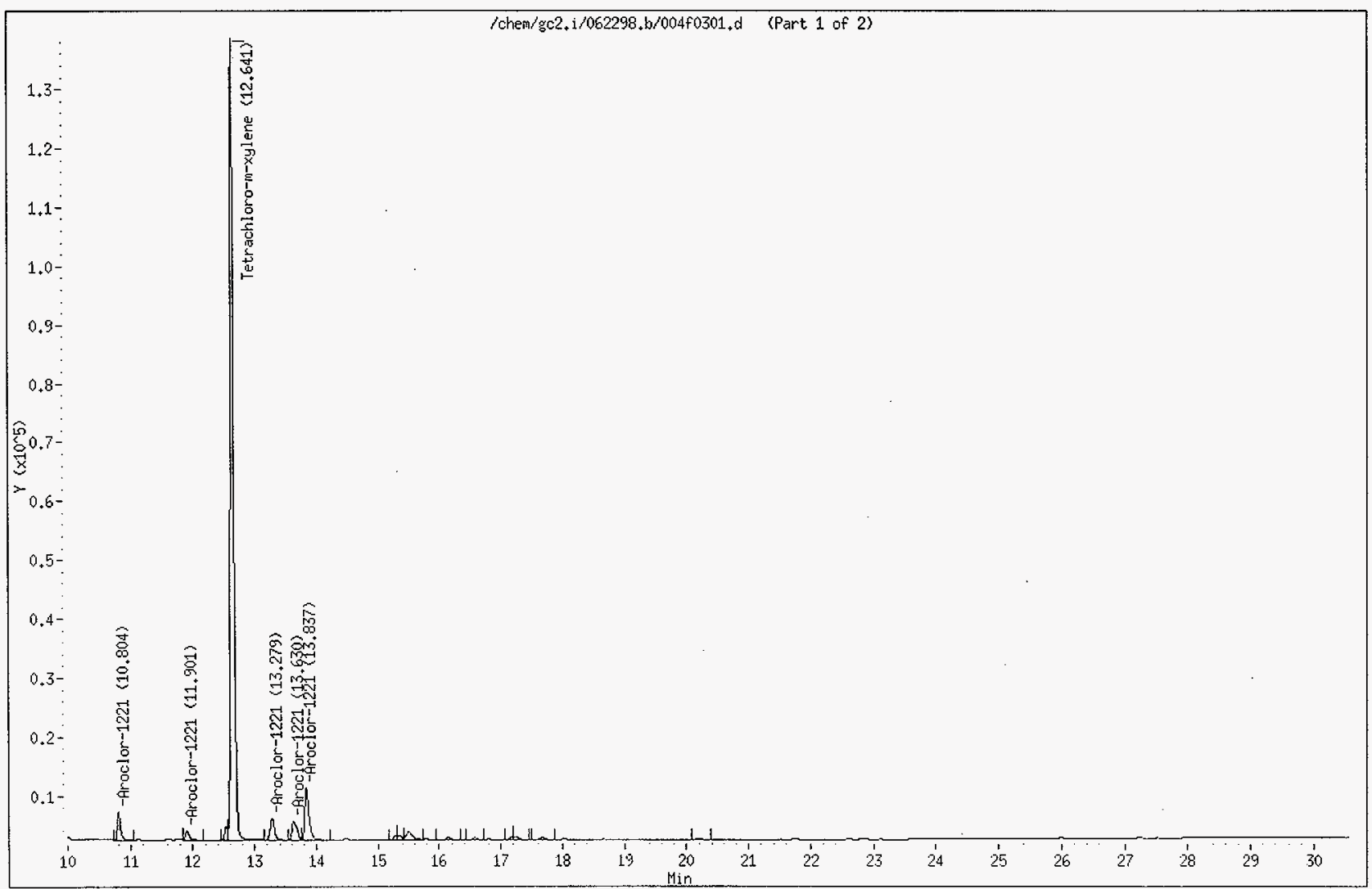


Data File: /chem/gc2,i/062298,b/004f0301,d

Date : 22-3UN-98 17:05

Client ID: 400ng/ml A1221

Instrument: gc2.i

Sample Info: 400ng'ml A1221

Volume Injected (uL) $\div 1.0$

Operator: Gerald Ross

Column. phase: $X \mathrm{ti}-5$

Column diameter* 0,25

/chem/gc2,i/062298,b/004f0301,d (Part. 2 of 2)

$\rightarrow$ की

$\omega$

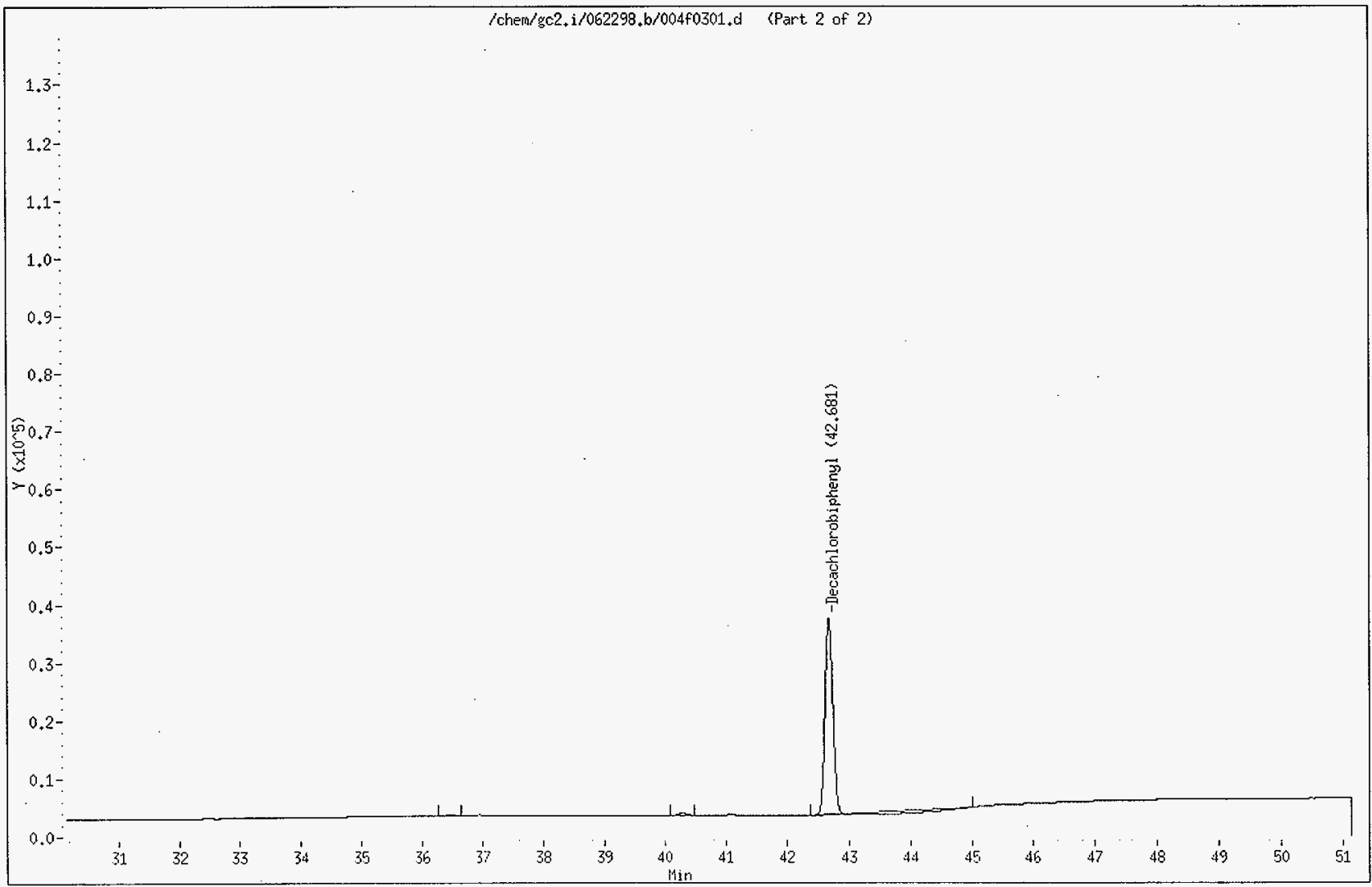




\section{HNF-SD-WM-DP-309 REV. 0}

Data File: /chem/gc2.i/062298.b/005f0301.d

Page 1

Report Date: 29-Jun-1998 13:37

\section{2-S Laboratory}

Data file : /chem/gc2.i/062298.b/005f0301.d

Lab Smp Id: $200 \mathrm{ng} / \mathrm{ml}$ A1221

Inj Date : 22-JUN-98 18:03

Operator : Gerald Ross

Smp Info : $200 \mathrm{ng} / \mathrm{ml}$ A1221

Misc Info : $200 \mathrm{ng} / \mathrm{ml}$ A1221

comment :

Method : /chem/gc2.i/062298.b/rcrapcb.m

Meth Date : 24-Jun-1998 09:58 gar

Cal Date : 22-JUN-98 18:03

Als bottle: 1

Dil Factor: 1.000

Integrator: HP Genie

Target Version: 3.10
Client Smp ID: $200 \mathrm{ng} / \mathrm{ml}$ A1221

Inst ID: $g \mathrm{c} 2 . \mathrm{i}$

Quant Type: ESTD

Cal File: 005f0301.d

calibration Sample, Level: 3

Compound Sublist: AR1221.sub

Sample Matrix: WATER

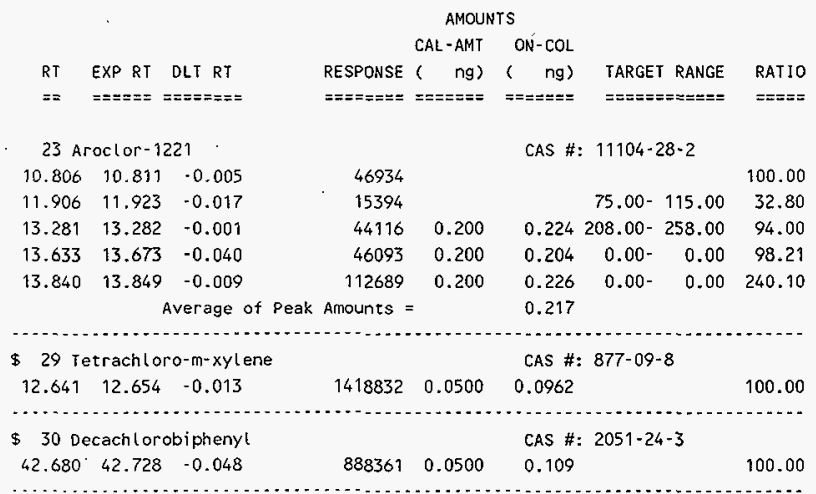


Data File: /chem/gc2.i/062298.b/005f0301.d

Report Date: 29-Jun-1998 13:37

$$
\begin{aligned}
& \text { 222-S Laboratory } \\
& \text { TARGET COMPOUNDS }
\end{aligned}
$$

Client Name:

Lab Smp Id: $200 \mathrm{ng} / \mathrm{ml}$ A1221

Sample Location:

Sample Date:

Sample Matrix: WATER

Analysis Type: PEST

Data Type: GC MULTI COMP

Misc Info: $200 \mathrm{ng} / \mathrm{ml} \mathrm{A} 1221$
Client SDG: 062298

Client Smp ID: $200 \mathrm{ng} / \mathrm{ml}$ A1221

Sample Point:

Date Received:

Quant Type: ESTD

Level: LOW

Operator: Gerald Ross

CONCENTRATION UNITS:

CAS NO.

COMPOUND

( $\mathrm{ug} / \mathrm{L}$ or $\mathrm{ug} / \mathrm{KG}$ ) ug/L

4.35

11104-28-2--.-Aroclor-1221

$======\begin{array}{r}4.35 \\ 1.92 \\ 2.18\end{array} \mid=====$


Date : 22-JuN-98 18:03

Client ID: 200ng/ml R1221

Sample Info: 200ng/ml A1221

Volume Injected $(u L\rangle: 1.0$

Instrument: gc2.i

Column phase: Xti-5

Dperator: Gerald Ross

Columir dianeter: 0.25

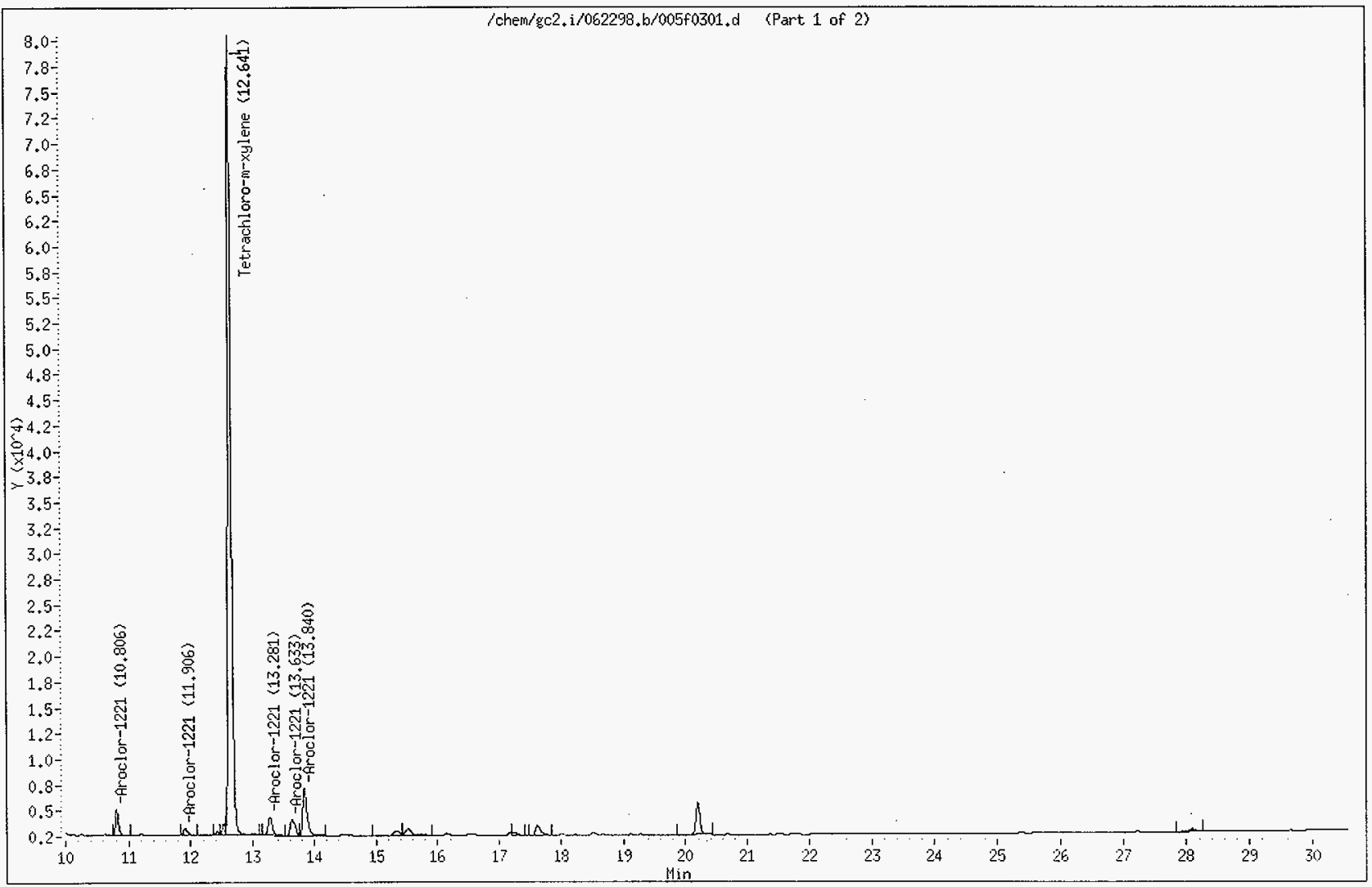

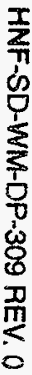


Data File: /chem/gc2,i/062298,b/005f0301.d

Page 4

late : 22-JuN-98 18:03

Client ID: 2000ng/ml 91221

Sample Info: 200ng/ml A1221

Volume Injected (uL): 1.0

Operator: Gerald Ross

Column phase: Xti-5

Column diameter: 0.25

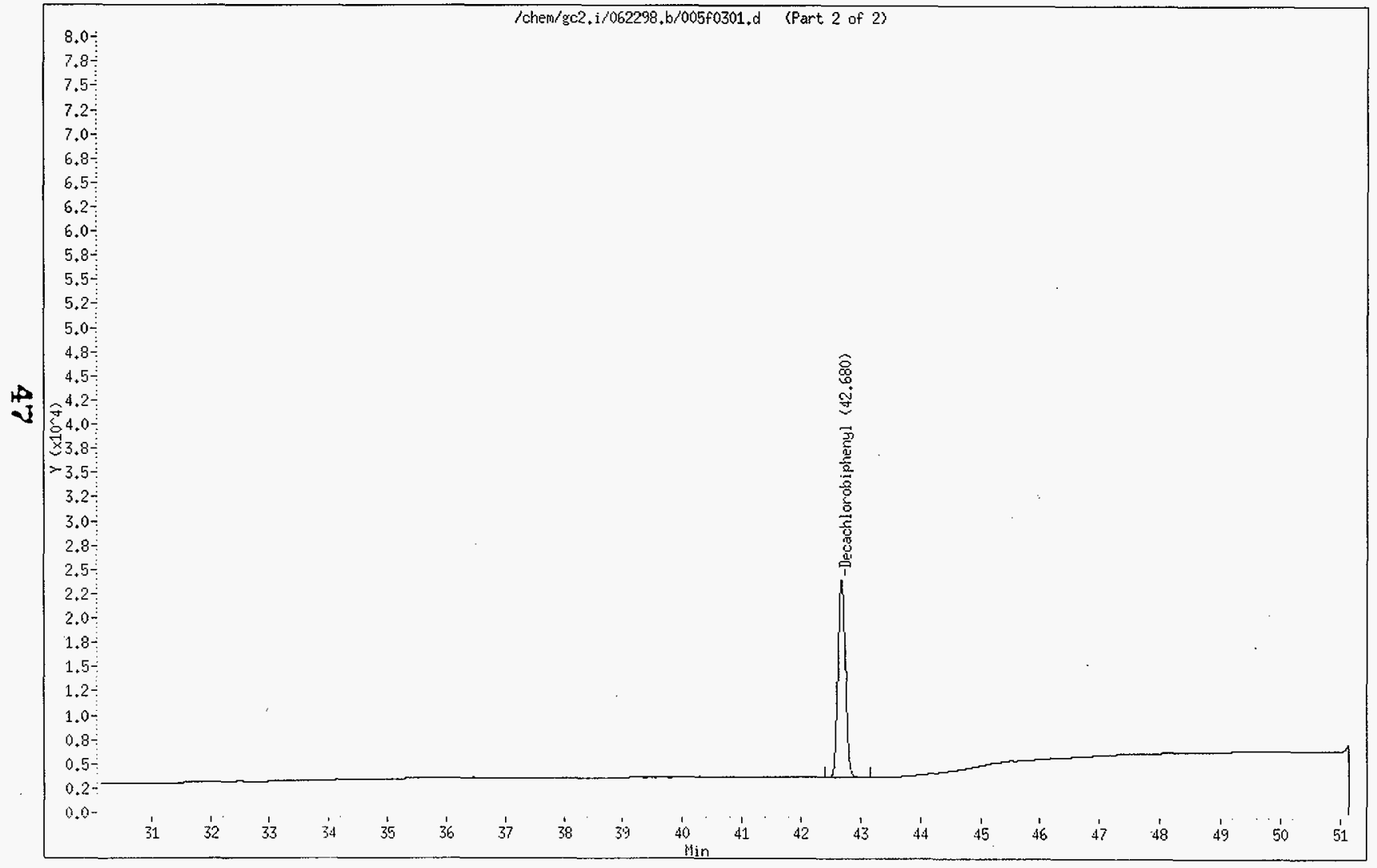

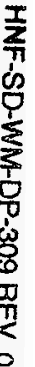




\section{2-S Laboratory}

Data file:/chem/gc2.i/062298.b/006f0301.d

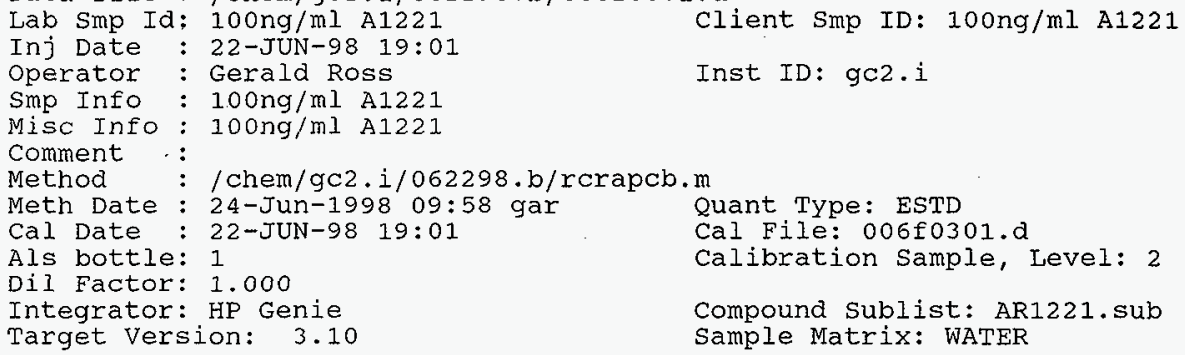

\begin{tabular}{|c|c|c|c|c|c|c|c|c|}
\hline & & & & AMOU: & & & & \\
\hline & & & & CAL - AMY & $\mathrm{ON}-\mathrm{COL}$ & & & \\
\hline RI & EXP RT & DLI RT & RESPONSE & ( $n g)$ & ( $n g)$ & TARGE T & T RANGE & RATIO \\
\hline$==$ & $=\Xi \pi \Xi=\#$ & $=======\Xi$ & $======\mathbf{z}$ & 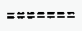 & $====\Xi \approx=$ & $=====$ & :=:==ニ= & $m===0$ \\
\hline - $23 \mathrm{Ar}$ & roclor-1 & 1221 & & & CAS \#: & $=11104-2$ & $28-2$ & \\
\hline 10.808 & 30.811 & $1-0.003$ & 19407 & & & & & $100.00(\mathrm{M})$ \\
\hline 11.913 & 11.923 & $3-0.010$ & 7709 & & & 75.00 & 115.00 & 39.72 \\
\hline 13.283 & 13.282 & 0.001 & 16439 & 0.100 & 0.0820 & $208.00-$ & 258.00 & 84.70 \\
\hline 13.672 & 13.673 & $=0.001$ & 23100 & 0.100 & 0.103 & 0.00 & 0.00 & 119.03 \\
\hline 13.845 & 13.849 & $\Rightarrow \quad-0.004$ & 47036 & 0.100 & 0.0933 & $0.00-$ & 0.00 & 242.36 \\
\hline & & Average 0 & Amounts $=$ & & 0.0944 & & & \\
\hline$\$ 29 \mathrm{Te}$ & etrachlo & oro-m-xyle & & & CAS \#: & : $877-09$ & & \\
\hline 12.642 & 12.654 & -0.012 & 1109398 & 0.0250 & 0.0752 & & & 100.00 \\
\hline$\$ 30 \mathrm{DE}$ & ecachlor & robiphenyl & & & CAS \#: & $=2051-24$ & $4-3$ & \\
\hline 42.684 & 42.728 & $3-0.044$ & 692033 & 0.0250 & 0.0851 & & & 100.00 \\
\hline
\end{tabular}

QC Flag Legend

M - Compound response manually integrated. 
Data File: /chem/gc2.i/062298.b/006f0301.d

Report Date: 29-Jun-1998 13:37

\section{2-S Laboratory \\ TARGET COMPOUNDS}

client Name:

Lab Smp Id: 100ng/ml A1221

Sample Location:

Sample Date:

Sample Matrix: WATER

Analysis Type: PEST

Data Type: GC MULTI COMP

Misc Info: $100 \mathrm{ng} / \mathrm{ml}$ A.1221
Client SDG: 062298

Client Smp ID: $100 \mathrm{ng} / \mathrm{ml}$ A1221

Sample point:

Date Received:

Quant Type: ESTD

Level: LOW

Operator: Gerald Ross

CONCENTRATION UNITS:

( $\mathrm{ug} / \mathrm{L}$ or $\mathrm{ug} / \mathrm{KG}$ ) ug/L

\begin{tabular}{|c|c|c|}
\hline $11104-28-2-m--$ Aroclor -1221 & 1.89 & \\
\hline 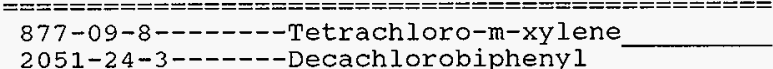 & $\begin{aligned}=-=z==== & ==2 \\
& 1.50 \\
& 1.70\end{aligned}$ & $===$ \\
\hline
\end{tabular}




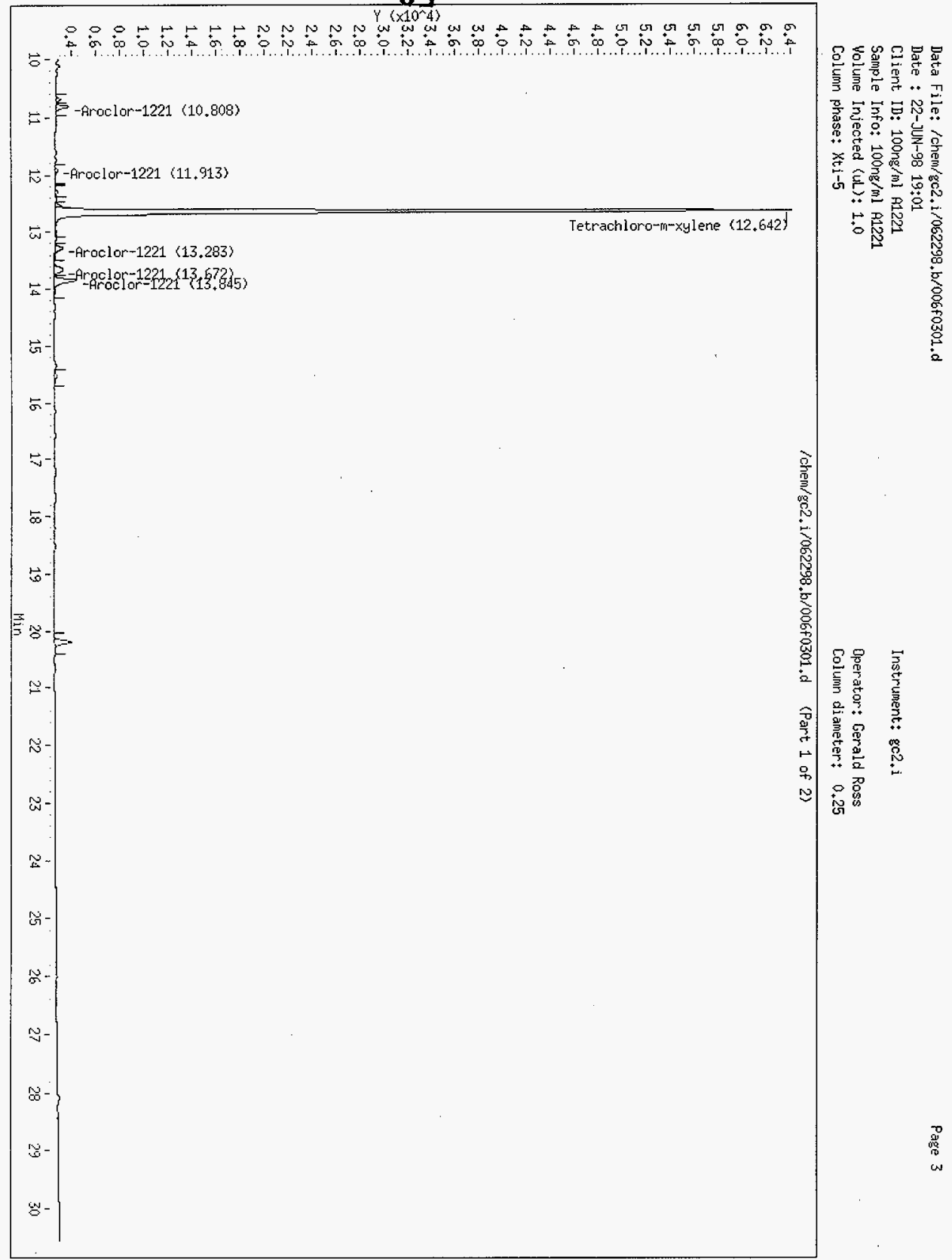


Data File: /chem/gc2, i/062298,b/006f0301.d

Date : 22-JuN-98 19*01

Client ID: $100 \mathrm{ng} / \mathrm{ml}$ A1221

Sample Info: 100ng/ml A1221

Volume In,jected $(u L\rangle): 1,0$

Instrungent: $\mathrm{gc}^{2}$

Column phase: Xti-5

Bperator: Gerald Ross

Columan diameter: 0.25

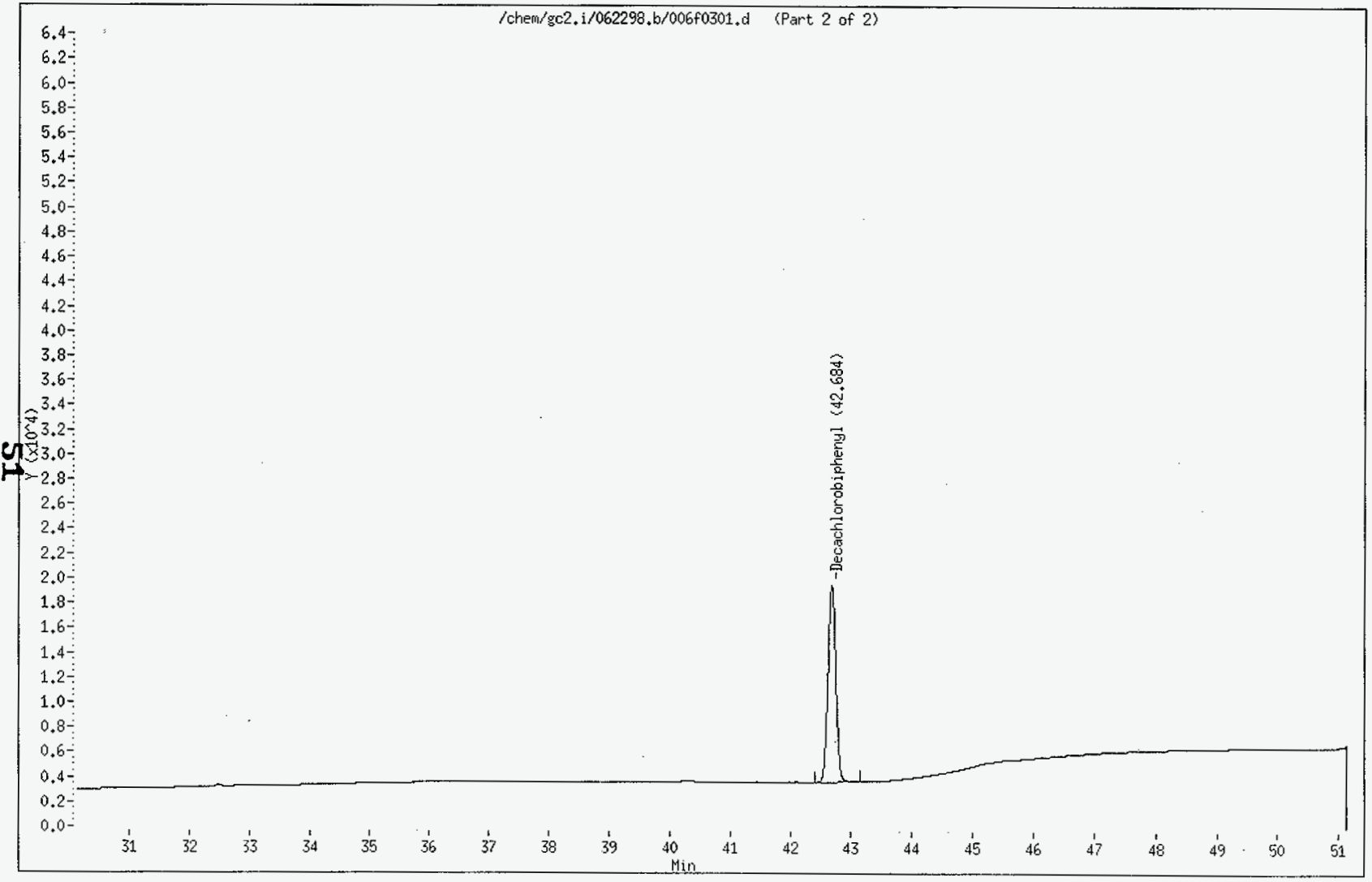

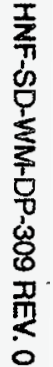


Data File: /chem/gc2.i/062298.b/007f0301.d

Report Date: 20-Jul-1998 09:08

\section{2-S Laboratory}

Data file : /chem/gc2.i/062298.b/007f0301.d

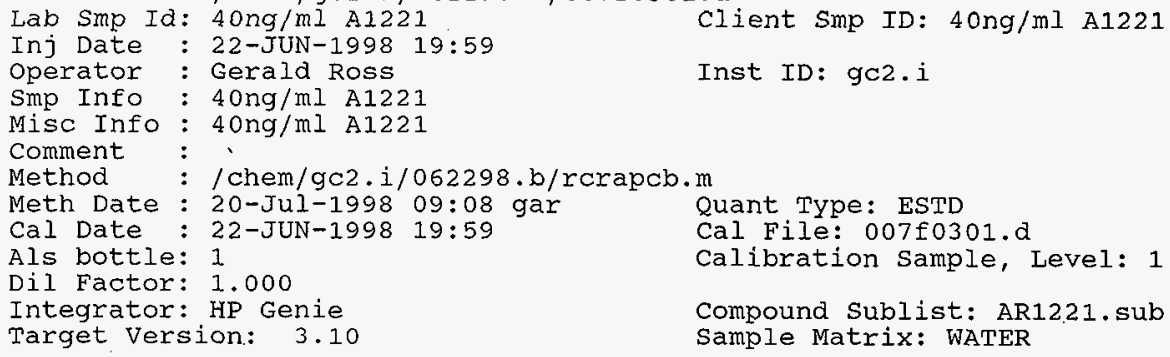

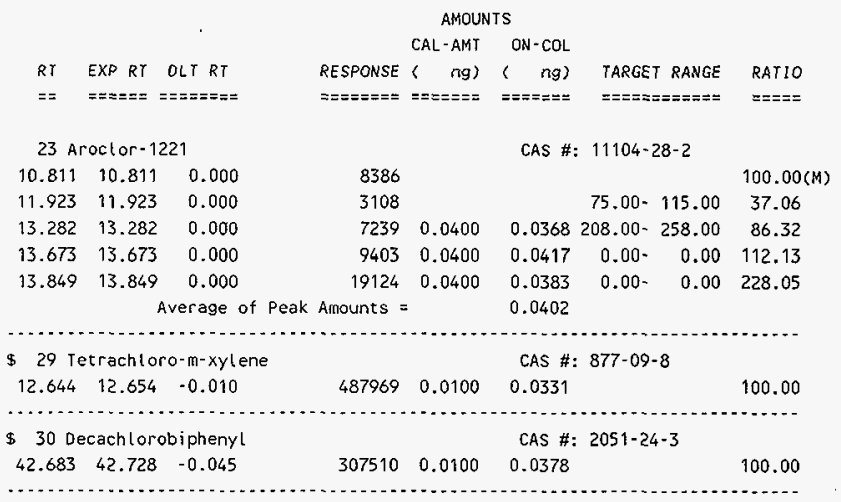

QC Flag Legend

M - Compound response manually integrated. 
Data File: /chem/gc2.i/062298.b/007f0301.d

Report Date: 20-Jul-1998 09:08

\section{2-s Laboratory}

TARGET COMPOUNDS

Client Name:

Lab Smp Id: 40ng/ml A1221

Sample Location:

Sample Date:

Sample Matrix: WATER

Analysis Type: PEST

Data Type: GC MULTI COMP

Misc Info: 4 ong/ml A1221
Client SDG: 062298

Client Smp ID: $40 \mathrm{ng} / \mathrm{ml}$ A1221

Sample Point:

Date Received:

Quant Type: ESTD

Level: LOW

Operator: Gerald Ross
CAS NO.

COMPOUND
CONCENTRATION UNITS: ( $\mathrm{ug} / \mathrm{L}$ or $\mathrm{ug} / \mathrm{KG}$ ) ug/I

\begin{tabular}{|c|c|c|}
\hline $11104-28-2-----$ Aroclor 1221 & $=========$ & $===$ \\
\hline $\begin{array}{l}877-09-8-\cdots-10 \text { Tetrachloro-m-xylene } \\
2051-24-3-\cdots--D e c a c h l o r o b i p h e n y l\end{array}$ & $\begin{array}{l}0.662 \\
0.756\end{array}$ & \\
\hline
\end{tabular}


Data File: /chem/ge2.i/062298,b/007f0301,d

bate : 22-JUN-1998 19:59

Client II * 40ng/ml A1221

Sample Info: 40ng/ml A1221

Volume Injected $(u L): 1,0$

Instrument: gc2.j

Column phase: Xti-5

Operator: Gerald Ross

Column diameter: 0.25

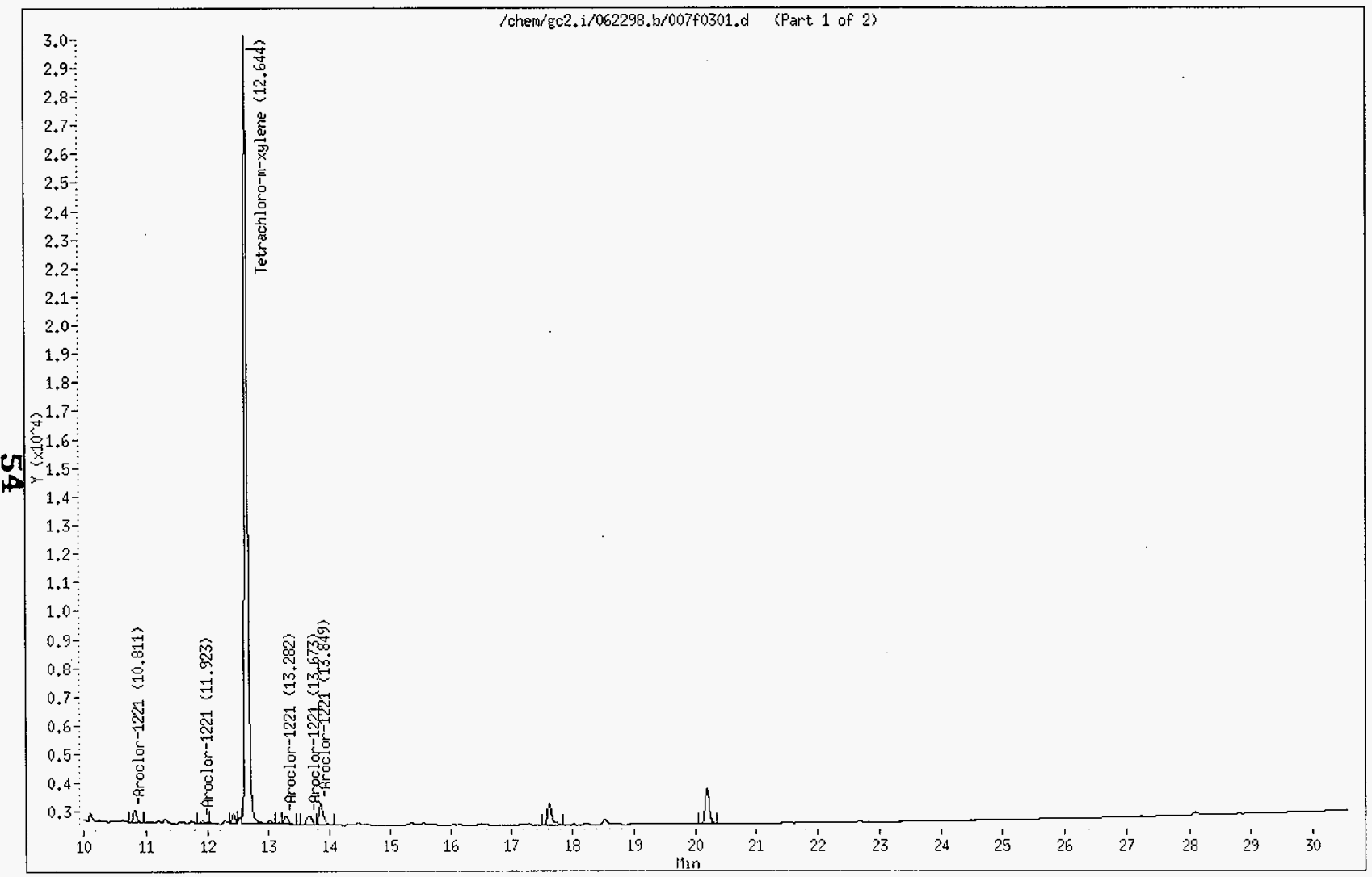




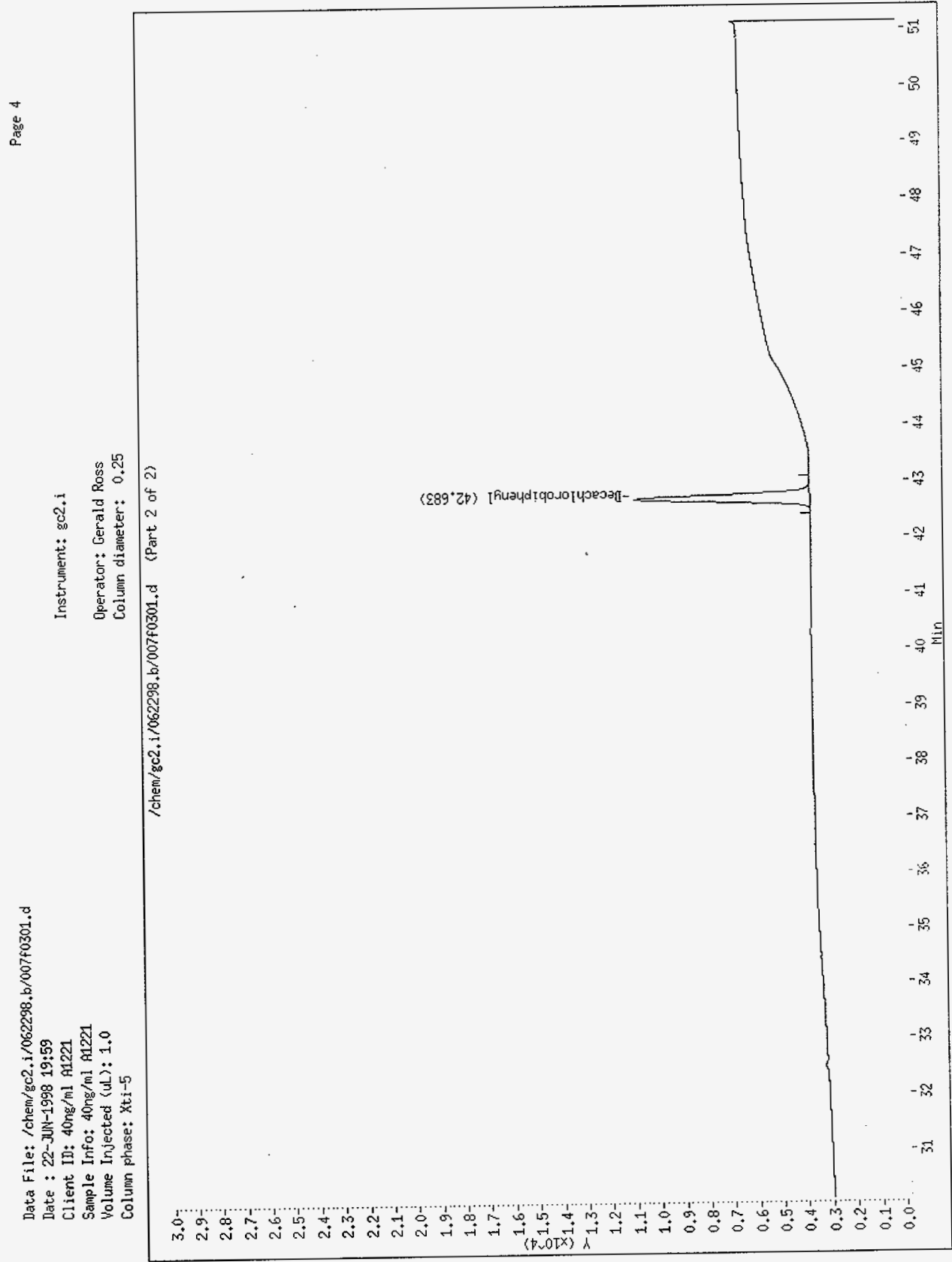

\section{5}


Data File: /chem/gc2.i/062298.b/008f0301.d

Report Date: 29-Jun-1998 13:38

Data file: /chem/gc2.i/062298.b/008f0301.d

Lab Smp Id: $200 \mathrm{ng} / \mathrm{ml}$ A1232

Inj Date : 22-JUN-98 20:57

Operator : Gerald Ross

Smp Info: $200 \mathrm{ng} / \mathrm{ml} \mathrm{A} 1232$

Client Smp ID: 200ng/ml A1232

Misc Info : $200 \mathrm{ng} / \mathrm{ml} \mathrm{A1232}$

Comment

Method : /chem/gc2:i/062298.b/rcrapcb.m

Meth Date : 24-Jun-1998 09:58 gar Quant Type: ESTD

Cal Date : 22-JUN-98 19:59

Inst ID: $g \mathrm{c} 2 . \mathrm{i}$

Als bottle: 1

Dil Factor: 1.000

Integrator: HP Genie

Target version: 3.10

Cal File: $007 f 0301 . d$

Compound sublist: AR1232.sub Sample Matrix: WATER

\begin{tabular}{|c|c|c|c|c|c|c|c|}
\hline \multicolumn{8}{|c|}{ CONCENTRATIONS } \\
\hline \multirow[b]{2}{*}{ RT } & \multirow{2}{*}{\multicolumn{2}{|c|}{ EXP RT OLT RT }} & \multirow[b]{2}{*}{ RESPONSE } & $\mathrm{ON}-\mathrm{COL}$ & \multicolumn{2}{|l|}{ FINAL } & \multirow[b]{2}{*}{ RATIO } \\
\hline & & & & ( $n g)$ & $(\mathrm{ug} / \mathrm{L})$ & TARGET RANGE & \\
\hline$==$ & $= \pm=\Omega=\Omega$ & $===ニ==+$ & $n=\cong ニ====$ & $==\pi==\pi$ & $==-ニ ニ==$ & 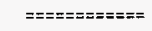 & $=\approx= \pm=$ \\
\hline \multicolumn{3}{|c|}{24 Aroclor-1232 } & & \multicolumn{4}{|c|}{ CAS \#: $1114-16-5$} \\
\hline 13.841 & 13.856 & -0.015 & 95866 & 0.183 & 3.67 & & 100.00 \\
\hline 15.356 & 15.376 & -0.020 & 55704 & 0.172 & 3.45 & $110.00-140.00$ & 58.11 \\
\hline 17.231 & 17.249 & -0.018 & 74987 & 0.182 & 3.65 & $126.00-166.00$ & 78.22 \\
\hline \multicolumn{4}{|c|}{ average of Peak cor } & \multicolumn{2}{|r|}{3.59} & & \\
\hline \multicolumn{4}{|c|}{$\$ 29$ Tetrachloro-m-xylene } & \multicolumn{4}{|c|}{ CAS \#: $877-09-8$} \\
\hline 12.641 & 12.654 & -0.013 & 1374661 & 0.0932 & 1.86 & . & 100.00 \\
\hline \multicolumn{4}{|c|}{ \$ 30 Decachlorobiphenyl } & \multicolumn{4}{|c|}{ CAS $\#: 2051-24-3$} \\
\hline 42.681 & 42.728 & .0 .047 & 731549 & 0.0899 & 1.80 & & 100.00 \\
\hline
\end{tabular}


Data File:/chem/gc2.i/062298.b/008f0301.d

Report Date: 29-Jun-1998 13:38

\section{2-s Laboratory}

\section{RECOVERY REPORT}

Client Name:

Sample Matrix: LIQUTD

Lab Smp Id: $200 \mathrm{ng} / \mathrm{ml}$ A1232

Level: LOW

Data Type: GC MULTI COMP

Spikelist File: RESOLUTION.spk

Method File: / chem/gc2.i/062298.b/rcrapcb.m

Misc Info: $200 \mathrm{ng} / \mathrm{ml}$ A 1232
Client SDG: 062298

Fraction: PEST

Client Smp ID: 200ng/ml A1232

operator: Gerald Ross

SampleType: SAMPLE

Quant Type: ESTD

\begin{tabular}{|lll|}
\hline SURROGATE COMPOUND \\
\hline$\$$ & 29 & Tetrachloro-m-xyle \\
$\$$ & 30 & Decachlorobiphenyl \\
\hline
\end{tabular}

\begin{tabular}{|l} 
CONC \\
ADDED \\
$\mathrm{ug} / \mathrm{L}$
\end{tabular}

\begin{tabular}{|} 
CONC \\
RECOVERED \\
Ug
\end{tabular}

\begin{tabular}{|c|} 
RECOVERED \\
\hline $\begin{array}{l}\frac{\%}{\circ} \\
89.92\end{array}$
\end{tabular}

\begin{tabular}{l} 
LIMITS \\
\hline $50-150$ \\
$50-150$ \\
\hline
\end{tabular}


Data File: /chem/gc2.i/062298.b/008f0301.d

Report Date: 29-Jun-1998 13:38

\section{2-S Laboratory \\ TARGET COMPOUNDS}

Client Name:

Lab Smp Id: $200 \mathrm{ng} / \mathrm{ml}$ A1232

Sample Location:

Sample Date:

Sample Matrix: WATER

Analysis Type: PEST

Data Type: GC MULTI COMP

Misc Info: $200 \mathrm{ng} / \mathrm{ml}$ A1232
Client SDG: 062298

Client Smp ID: $200 \mathrm{ng} / \mathrm{ml}$ A1232

Sample point:

Date Received:

Quant Type: ESTD

Level: LOW

Operator: Gerald Ross

CONCENTRATION UNITS:

( $\mathrm{ug} / \mathrm{L}$ or $\mathrm{ug} / \mathrm{KG}$ ) ug/L

\begin{tabular}{|c|c|c|}
\hline 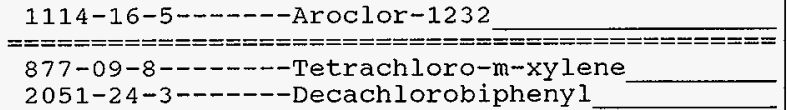 & $======\begin{array}{r}3.59 \\
1.86 \\
1.80\end{array}$ & $====$ \\
\hline
\end{tabular}


late : 22-JUN-98 20:57

Client ID: 200ng/ml A1232

Sample Info: $200 \mathrm{ng} / \mathrm{ml}$ A1232

Volume Injected $(u L): 1.0$

Instrument: gc2.i

Dperator: Cerald Ross

Column diameter: 0.25

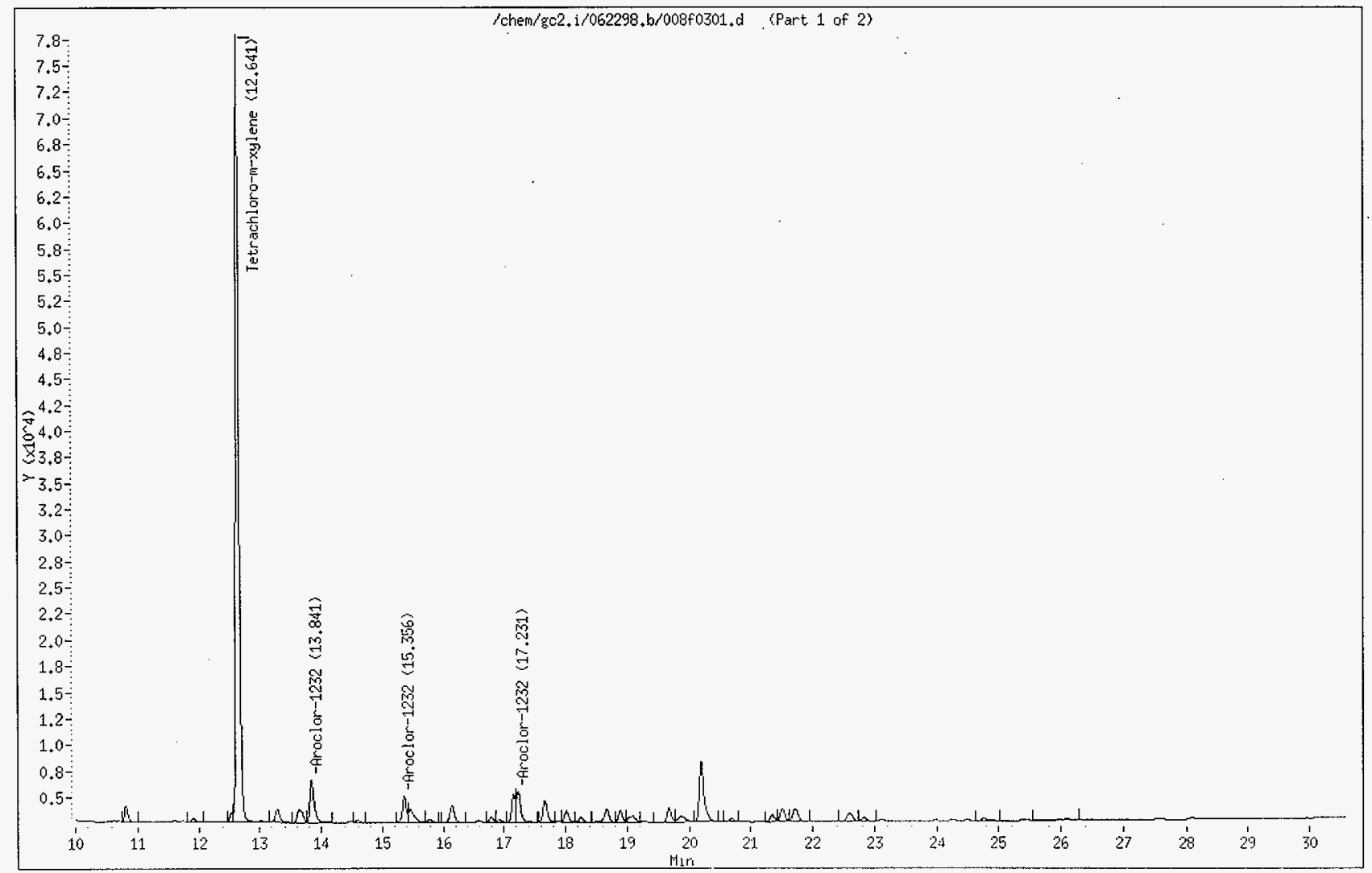


Data File: /chem/gc2. i/062298,b/008f0301,d

Date : 22-JUN-98 20+57

Client ID: 200ng/ml A1232

Sample Info: 200ng/ml A1232

Volume Injected $(u L) \div 1.0$

Instrument: gc2.j

Column phase: $X_{t i}-5$

Operator: Gerald Ross

Column diameter: 0.25

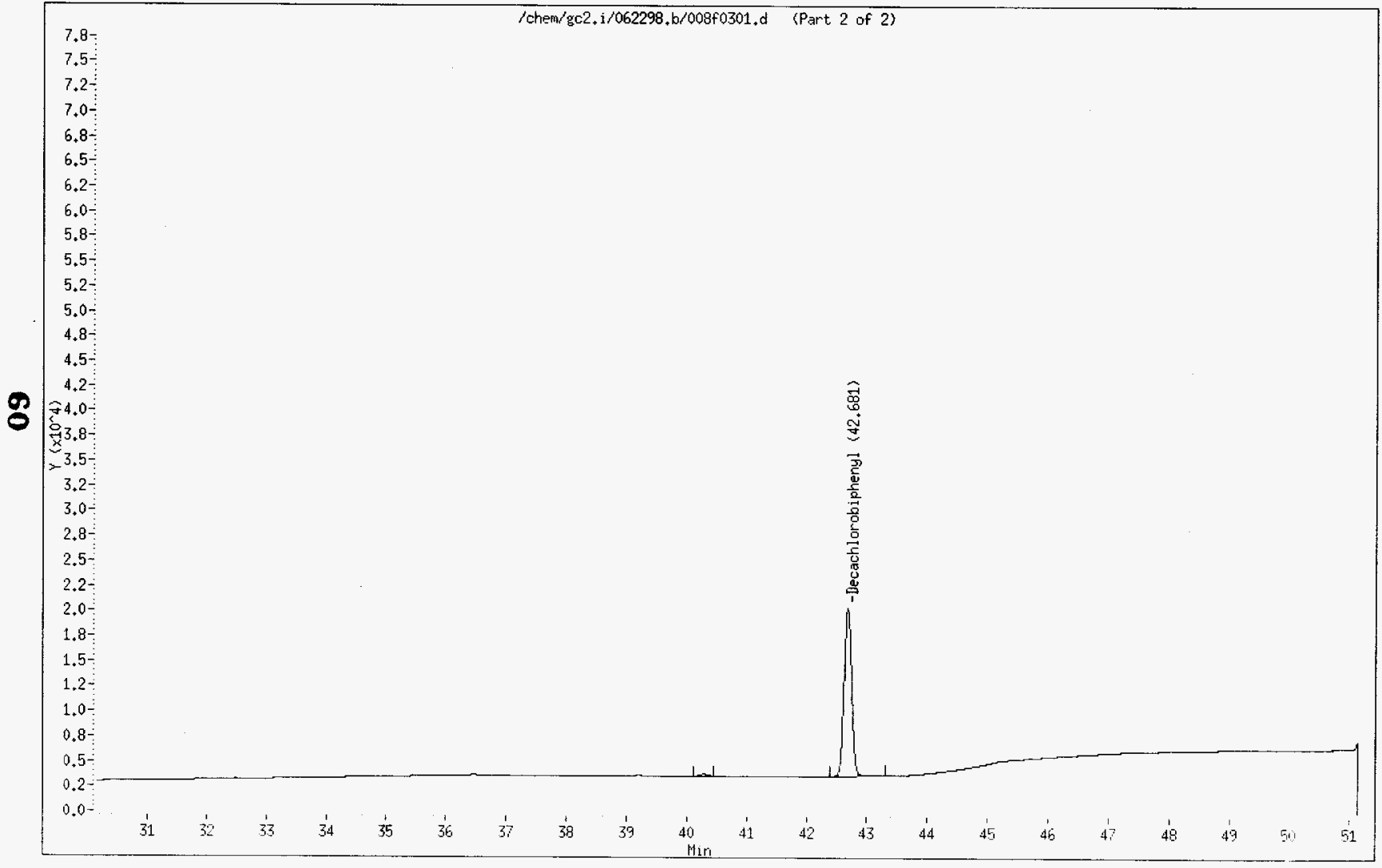


Data File: /chem/gc2.i/062298.b/009f0301.d

Report Date: 29-Jun-1998 13:38

222-S Laboratory

Data file:/chem/gc2.i/062298.b/009f0301.d

Lab Smp Id: $200 \mathrm{ng} / \mathrm{ml}$ A 1242

Inj Date : 22-JUN-98 21:55

Operator : Gerald Ross

Smp Info : $200 \mathrm{ng} / \mathrm{ml} \mathrm{A} 1242$

Misc Info: $200 \mathrm{ng} / \mathrm{ml}$ A12 42

Comment :

Method : /chem/gc2.i/062298.b/rcrapcb.m

Meth Date : 24-Jun-1998 09:58 gar

Cal Date : 22-JUN-98 19:59

Quant Type: ESTD

Als bottle: 1

Dil Factor: 1.000

Integrator: HP Genie

Target version: 3.10

Client Smp ID: $200 \mathrm{ng} / \mathrm{ml} \mathrm{A1242}$

Inst ID: $\mathrm{gc} 2 . \mathrm{i}$

Cal File: $007 f 0301 . d$

Compound Sublist: AR1242.sub

Sample Matrix: WATER

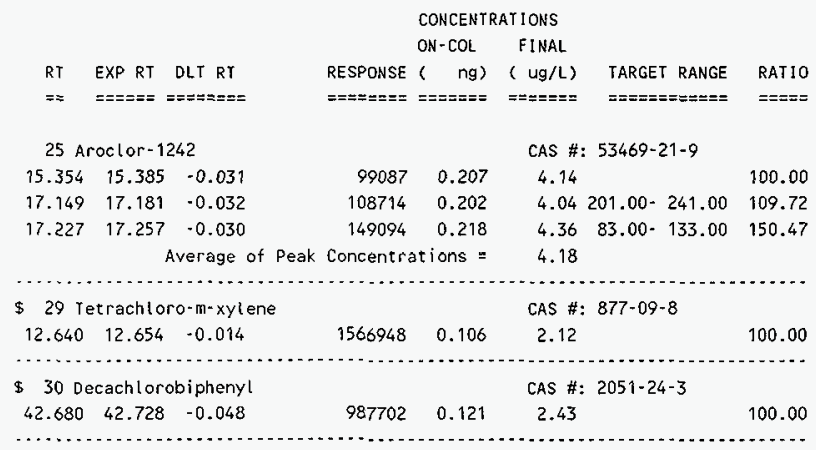


Data File: /chem/gc2.i/062298.b/009f0301.d

Report Date: 29-Jun-1998 13:38

$$
\text { 222-S Laboratory }
$$

\section{RECOVERY REPORT}

client Name:

Sample Matrix: LIQUID

Lab Smp Id: $200 \mathrm{ng} / \mathrm{ml}$ A1242

Leve 1: LOW

Data Type: GC MUITI COMP

SpikeIist File: RESOLUTION.spk

Method $\mathrm{File} / \mathrm{chem} / \mathrm{gc}^{2} \mathrm{i} / 062298$

$\mathrm{Misc}$ Info: $200 \mathrm{ng} / \mathrm{ml} \mathrm{A} 1242$

\begin{tabular}{|c|c|c|c|c|}
\hline SURROGATE COMPOUND & $\begin{array}{l}\text { CONC } \\
\text { ADDED }\end{array}$ & $\begin{array}{c}\text { CONC } \\
\text { RECOVERED }\end{array}$ & RECOVERED & LIMITS \\
\hline $\begin{array}{lll}\$ & 29 & \text { Tetrachloro-m-xyle } \\
\$ & 30 & \text { Decachlorobiphenyl }\end{array}$ & $\begin{array}{l}2.00 \\
2.00\end{array}$ & $\begin{array}{l}2.12 \\
2.43\end{array}$ & $\begin{array}{l}106.27 \\
121.41\end{array}$ & $\begin{array}{l}50-150 \\
50-150\end{array}$ \\
\hline
\end{tabular}

Client SDG: 062298

Fraction: PEST

Client Smp ID: 200ng/ml Al242

operator: Gerald Ross

SampleType: SAMPLE

Quant Type: ESTD b/rcrapcb.m 
Data File: /chem/gc2.i/062298.b/009f0301.d

Report Date: 29-Jun-1998 13:38

\section{2-S Laboratory \\ TARGET COMPOUNDS}

Client Name:

Lab Smp Id: $200 \mathrm{ng} / \mathrm{ml}$ A1242

Sample Location:

Sample Date:

Sample Matrix: WATER

Analysis Type: PEST

Data Type: GC MULTI COMP

Misc Info: $200 \mathrm{ng} / \mathrm{ml}$ A1242
Client SDG: 062298

Client Smp ID: $200 \mathrm{ng} / \mathrm{ml} \mathrm{A1242}$

Sample Point:

Date Received:

Quant Type: ESTD

Level: LOW

operator: Gerald Ross

CONCENTRATION UNITS:

(ug/L or $\mathrm{ug} / \mathrm{KG}$ ) ug/L

\begin{tabular}{|c|c|c|}
\hline $53469-21-9------A r o c 1$ or -1242 & 4.18 & $===\overline{=}$ \\
\hline $\begin{array}{l}877-09-8-1 \\
2051-24-3------D e c a c h l o r o b i p h e n y l\end{array}$ & $\begin{array}{r}2.12 \\
2.43\end{array}$ & \\
\hline
\end{tabular}


Client ID: $200 \mathrm{ng} / \mathrm{ml} \mathrm{A1242}$

Sample Info: $200 \mathrm{ng} / \mathrm{ml}$ A1242

Volume Injected (uL): 1.0

Coluran phase: $\mathrm{Xti}-5$

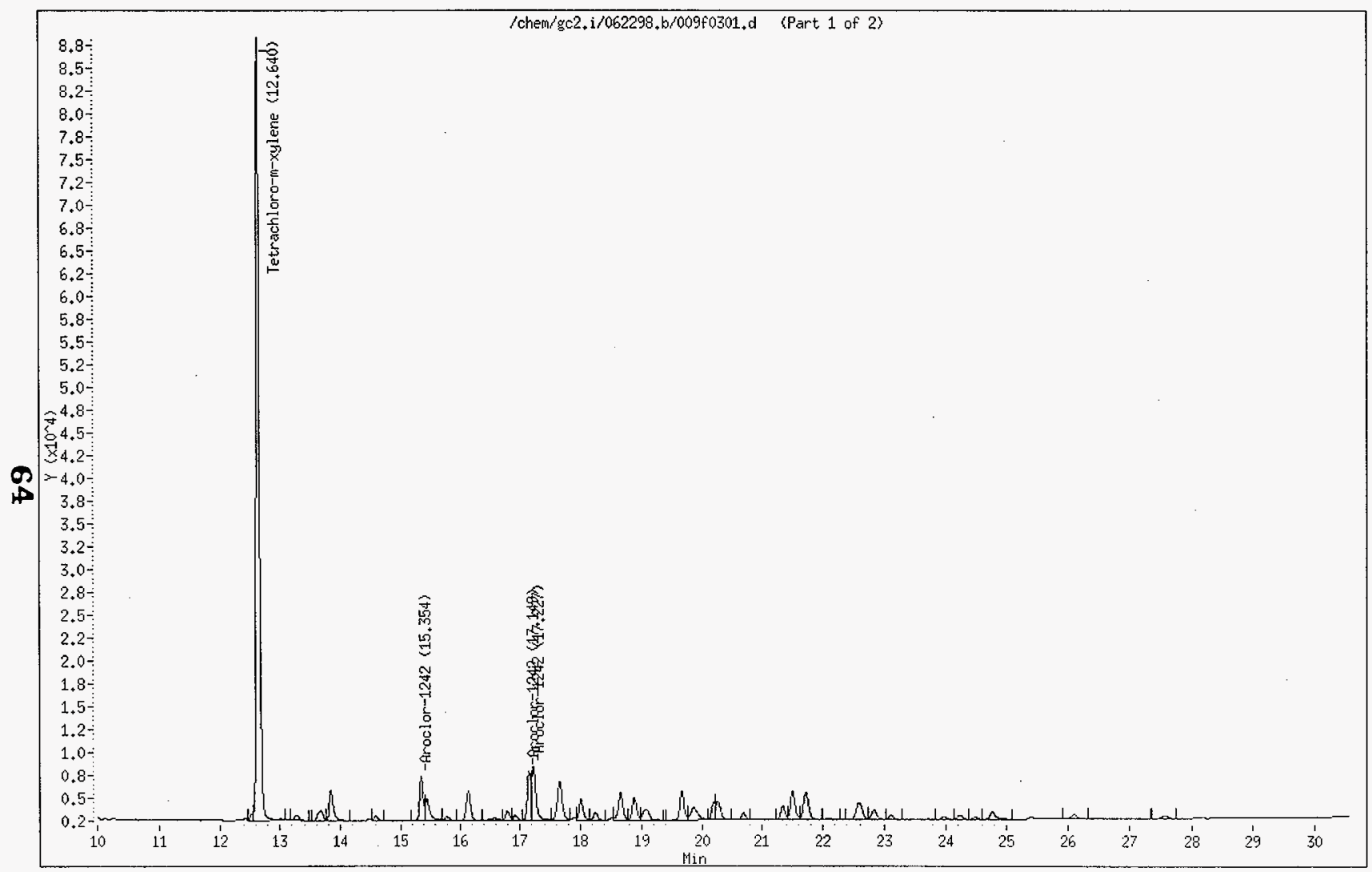

Instrument: gc2, i

Operator: Gerald Ross
Operator: Gerald Ross 
Data File: /chem/gc2,i/062298,b/009f0301.d

Date : 22-JuN-98 21:55

Instrument: $g c 2, i$

i: 200ng/mal A12

Operator: Gerald Ross

Yolume Injected (uL) $\$ 1.0$

Columin diameter: 0.25

Column phase+ $/$ ti-5

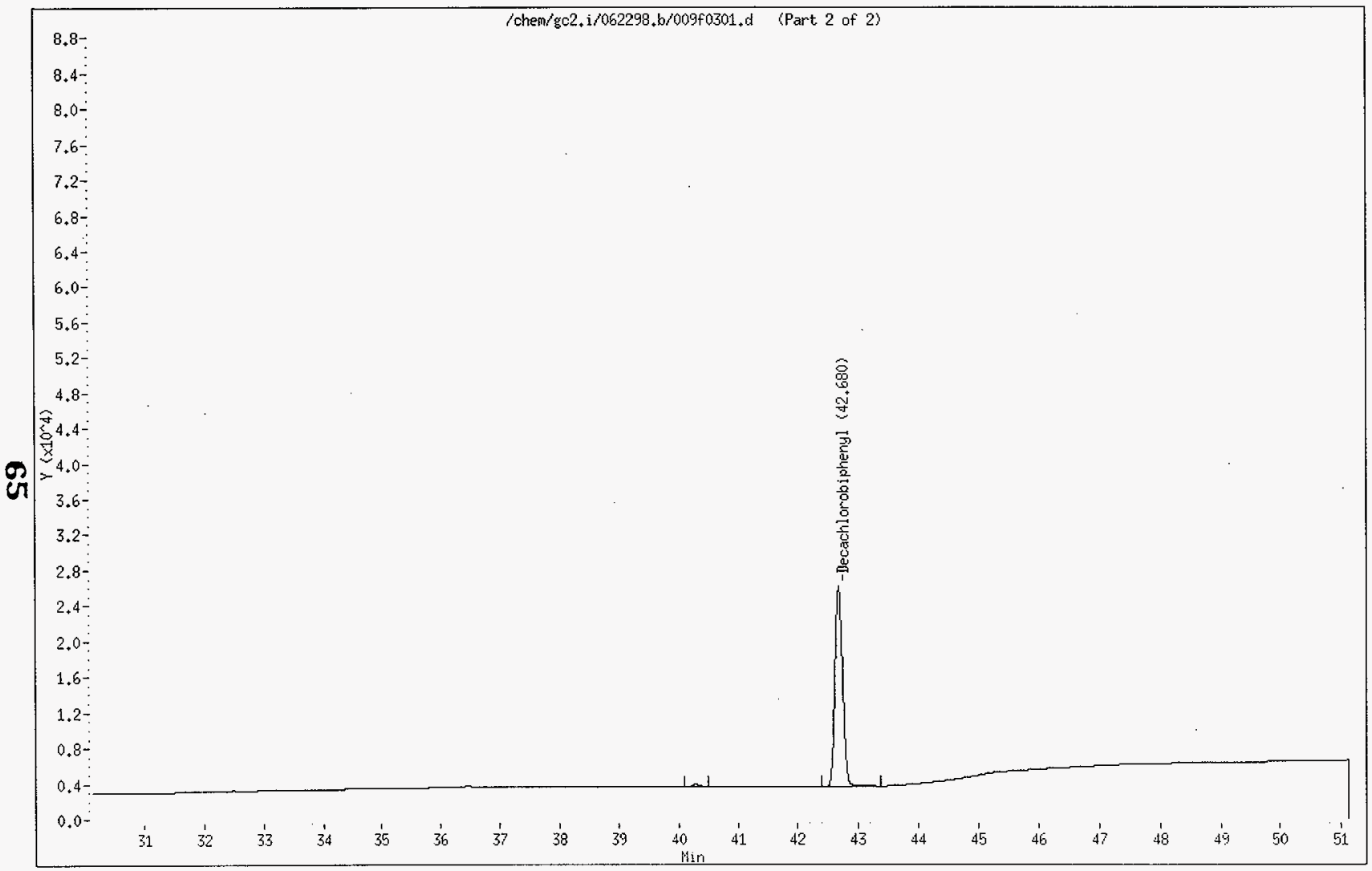


Data File: /chem/gc2.i/062298.b/010f0301.d

Report Date: 29-Jun-1998 13:38

\section{2-S Laboratory}

Data file : /chem/gc2.i/062298.b/010f0301.d

Lab Smp Id: 200ng/ml A1248

Inj Date : 22-JUN-98 22:53

operator : Gerald Ross

Smp Info : 200ng/ml A1248

Misc Info : $200 \mathrm{ng} / \mathrm{ml}$ A1248

comment :

Method : /chem/gc2.i/062298.b/rcrapcb.m

Meth Date : 24-Jun-1998 09:58 gar Quant Type: ESTD

Cal Date: 22-JUN-98 19:59

Als bottle: 1

Dil Factor: 1.000

Integrator: HP Genie

Target Version: 3.10 Sample Matrix: WATER
Client Smp ID: $200 \mathrm{ng} / \mathrm{ml}$ A1248

Inst ID: $g c 2 . i$

Compound Sublist: AR1248.sub

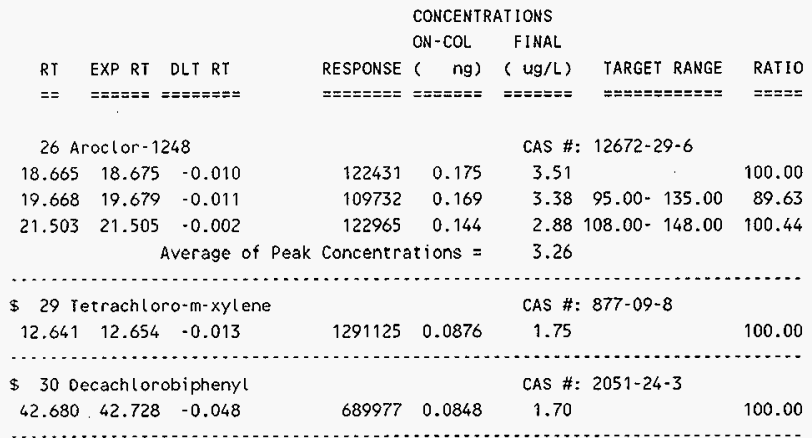


Data File: /chem/gc2.i/062298.b/010f0301.d

Report Date: 29-Jun-1998 13:38

$$
\text { 222-S Laboratory }
$$

RECOVERY REPORT

Client Name:

Sample Matrix: LIQUID

Lab Smp Id: 200ng/ml A1248

Level: LOW

Data Type: GC MULTI COMP

Spikelist File: RESOLUTION.spk

Method File: /chem/gc2.i/062298.b/rcrapcb.m

Misc Info: $200 \mathrm{ng} / \mathrm{ml} \mathrm{A} 1248$

\begin{tabular}{|c|c|c|c|c|}
\hline SURROGATE COMPOUND & $\begin{array}{l}\text { CONC } \\
\text { ADDED }\end{array}$ & $\begin{array}{c}\text { CONC } \\
\text { RECOVERED }\end{array}$ & $\begin{array}{c}\% \\
\text { RECOVERED }\end{array}$ & LIMITS \\
\hline $\begin{array}{lll}\$ & 29 & \text { Tetrachloro-m-xyle } \\
\$ & 30 & \text { Decachlorobiphenyl }\end{array}$ & $\begin{array}{l}2.00 \\
2.00\end{array}$ & $\begin{array}{l}1.75 \\
1.70\end{array}$ & $\begin{array}{l}87.56 \\
84.81\end{array}$ & $\begin{array}{l}50-150 \\
50-150\end{array}$ \\
\hline
\end{tabular}


Data File:/chem/gc2.i/062298.b/010f0301.d

Report Date: 29-Jun-1998 13:38

\section{2-S Laboratory}

TARGET COMPOUNDS

Client Name:

Lab Smp Id: $200 \mathrm{ng} / \mathrm{ml}$ A 1248

Sample Location:

Sample Date:

Sample Matrix: WATER

Analysis Type: PEST

Data Type: GC MULTI COMP

Misc Info: $200 \mathrm{ng} / \mathrm{ml}$ A1248
Client SDG: 062298

Client Smp ID: $200 \mathrm{ng} / \mathrm{ml}$ A1248

Sample Point:

Date Received:

Quant Type: ESTD

Level: LOW

operator: Gerald Ross

CONCENTRATION UNITS:

(ug/L or $\mathrm{ug} / \mathrm{KG}$ ) ug/L

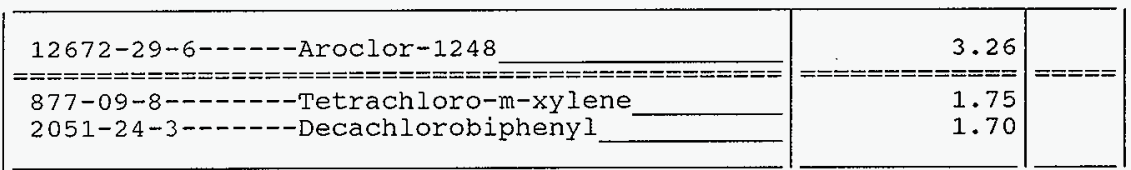


Ilata File: /chem/gc2, i/062298.b/010f0301,d

Date : 22-JuN-98 22:53

Page 5

Client ID: 200ng/ml A1248

Sample Info: 200ing/ml A1248

Volume Injected (uL): 1,0

Instrument: $\mathrm{gc2}+\mathrm{i}$

Column phase: $\mathrm{xti-5}$

Operator: Cerald Ross

Columin diameter: 0.25

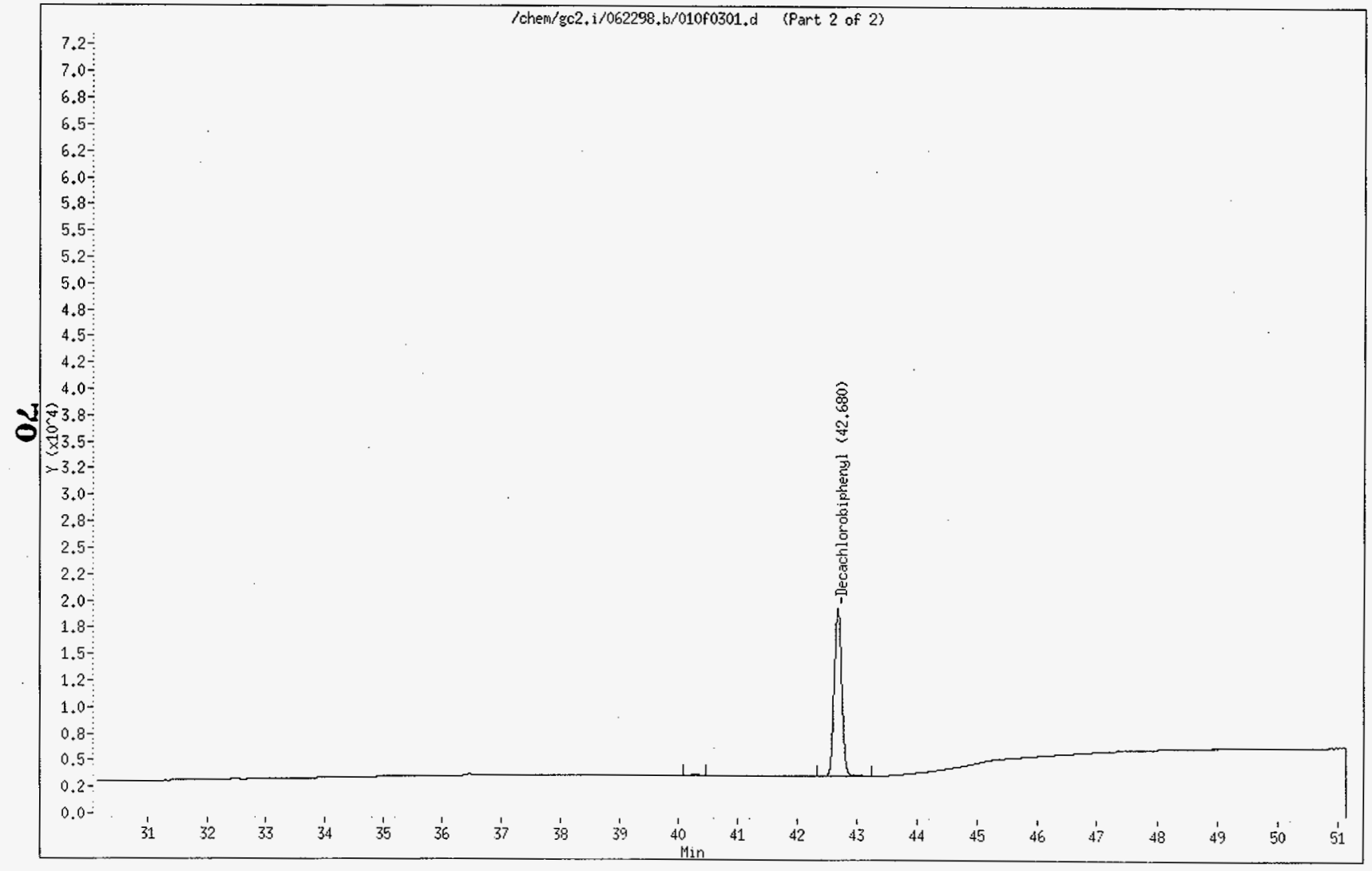




\section{HNF-SD-WM-DP-309 REV. 0}

Data File: /chem/gc2.i/062298.b/011f0301.d

Report Date: 29-Jun-1998 13:38

\section{2-S Laboratory}

Data file: /chem/gc2.i/062298.b/011f0301.d

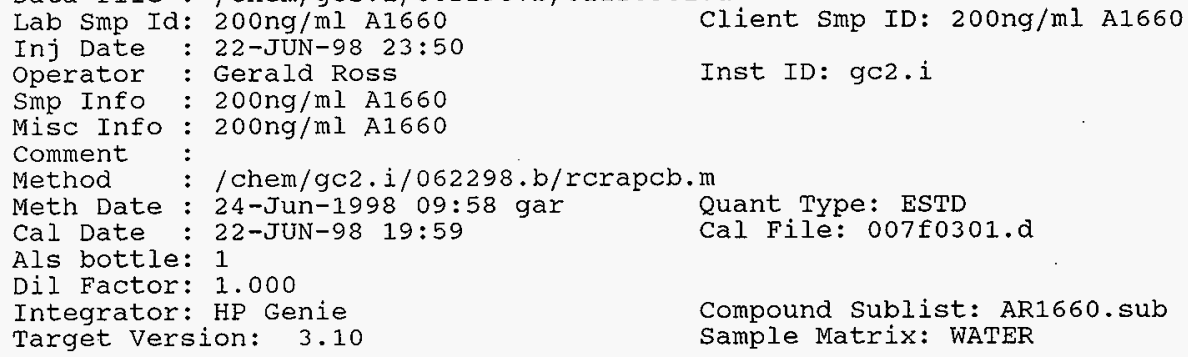

\begin{tabular}{|c|c|c|c|c|c|c|c|}
\hline & & & & CONCENTR & A.TIONS & & \\
\hline & & & & $\mathrm{ON}-\mathrm{COL}$ & FINAL & & \\
\hline$R T$ & EXP RT & DLT RT & RESPONSE & ( $n g$ ) & $(u g / L)$ & TARGET RANGE & RATIO \\
\hline$=\bar{x}$ & $=== \pm==$ & $=======$ & 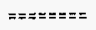 & $======8$ & $==\pi===$ & 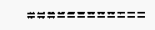 & $=====$ \\
\hline $22 \mathrm{Ar}$ & oclor -10 & 016 & & & CAS \#: & $=12674-11-2$ & \\
\hline 15.352 & 15.374 & -0.022 & 113954 & 0.176 & 3.52 & & 100.00 \\
\hline 17.147 & 17.171 & -0.024 & 125349 & 0.170 & 3.40 & $114.00-134.00$ & 110.00 \\
\hline 17.224 & 17.247 & -0.023 & 172862 & 0.183 & 3.66 & $134.00-154.00$ & 151.69 \\
\hline & & Average of Peak & Concentra & ations = & 3.53 & & \\
\hline $28 \mathrm{Ar}$ & oclor-12 & 260 & & & CAS \#: & : $11096-82-5$ & \\
\hline 25.989 & 26.019 & -0.030 & 173459 & 0.178 & 3.56 & & 100.00 \\
\hline 27.222 & 27.253 & -0.031 & 172663 & 0.178 & 3.57 & $89.00-109.00$ & 99.54 \\
\hline 28.770 & 28.800 & -0.030 & 164746 & 0.186 & 3.72 & $72.00-92.00$ & 94.98 \\
\hline & & Average of Peak & Concentra & ations $=$ & 3.61 & & \\
\hline$\$ 29 \mathrm{Te}$ & trachlor & ro-n-xylene & & & CAS \#: & $=877-09-8$ & \\
\hline 12.640 & 12.654 & -0.014 & 1491505 & 0.101 & 2.02 & & 100.00 \\
\hline$\$ 30 \mathrm{De}$ & cachlor & obiphenyt & & & CAS \#: & $2051-24-3$ & \\
\hline 42.679 & 42.728 & -0.049 & 829945 & 0.102 & 2.04 & & 100.00 \\
\hline
\end{tabular}


Data File:/chem/gc2.i/062298.b/011f0301.d

Report Date: 29-Jun-1998 13:38

$$
\text { 222-S Iaboratory }
$$

RECOVERY REPORT

Client Name:

Sample Matrix: LIQUID

Lab Smp Id: $200 \mathrm{ng} / \mathrm{ml}$ A1660

Level: LOW

Data Type: GC MULTI COMP

Spikelist File: RESOLUTION.spk

Method File: / chem/gc2.i/062298.b/rcrapcb.m

Misc Info: $200 \mathrm{ng} / \mathrm{mI}$ A1660

Client SDG: 062298

Fraction: PEST

Client Smp ID: 200ng/ml A1660

Operator: Gerald Ross

SampleTYpe: SAMPLE

Quant TYpe: ESTD

\begin{tabular}{|c|c|c|c|c|}
\hline SURROGATE COMPOUND & $\begin{array}{l}\text { CONC } \\
\text { ADDED } \\
\text { ug/L }\end{array}$ & $\begin{array}{c}\text { CONC } \\
\text { RECOVERED } \\
\text { ug/ L }\end{array}$ & $\stackrel{\circ}{\circ}$ RECOVERED & LIMITS \\
\hline $\begin{array}{lll}\$ & 29 & \text { Tetrachloro-m-xyle } \\
\$ & 30 & \text { Decachlorobiphenyl }\end{array}$ & $\begin{array}{l}2.00 \\
2.00\end{array}$ & $\begin{array}{l}2.02 \\
2.04\end{array}$ & $\begin{array}{l}101.15 \\
102.02\end{array}$ & $\begin{array}{l}50-150 \\
50-150\end{array}$ \\
\hline
\end{tabular}


Data File: /chem/gc2.i/062298.b/011f0301.d

Report Date: 29-Jun-1998 13:38

$$
\begin{aligned}
& \text { 222-S Laboratory } \\
& \text { TARGET COMPOUNDS }
\end{aligned}
$$

Client Name:

Lab Smp Id: $200 \mathrm{ng} / \mathrm{ml}$ A1660

Sample Location:

Sample Date:

Sample Matrix: WATER

Analysis Type: PEST

Data Type: GC MULTI COMP

Misc Info: $200 \mathrm{ng} / \mathrm{ml}$ A 1660
Client SDG: 062298

Client Smp ID: $200 \mathrm{ng} / \mathrm{ml}$ A1660

Sample Point:

Date Received:

Quant Type: ESTD

Leve1: LoW

Operator: Gerald Ross

CONCENTRATION UNITS:

( $\mathrm{ug} / \mathrm{L}$ or $\mathrm{ug} / \mathrm{KG}) \mathrm{ug} / \mathrm{L}$

\begin{tabular}{|c|c|c|}
\hline $\begin{array}{l}12674-11-2 \cdots---A r o c l o r-1016 \\
11096-82-5-\cdots-- \text { Aroclor }-1260\end{array}$ & $\begin{array}{l}3.53 \\
3.61\end{array}$ & \\
\hline 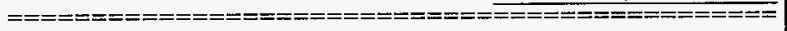 & 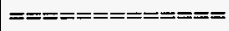 & $====$ \\
\hline $\begin{array}{l}877-09-8-1-0-T e t r a c h l o r o-m-x y l e n e \\
2051-24-3----D e c a c h l o r o b i p h e n y l\end{array}$ & $\begin{array}{r}2.02 \\
2.04\end{array}$ & \\
\hline
\end{tabular}


Date : 22-JUN-98 23:50

Client ID: 200ng/ml A1660

Sample Info: $200 \mathrm{ng} / \mathrm{ml}$ A1660

Volume Injected (uL): 1.0

Operator: Gerald Ross

Column diameter: 0.25

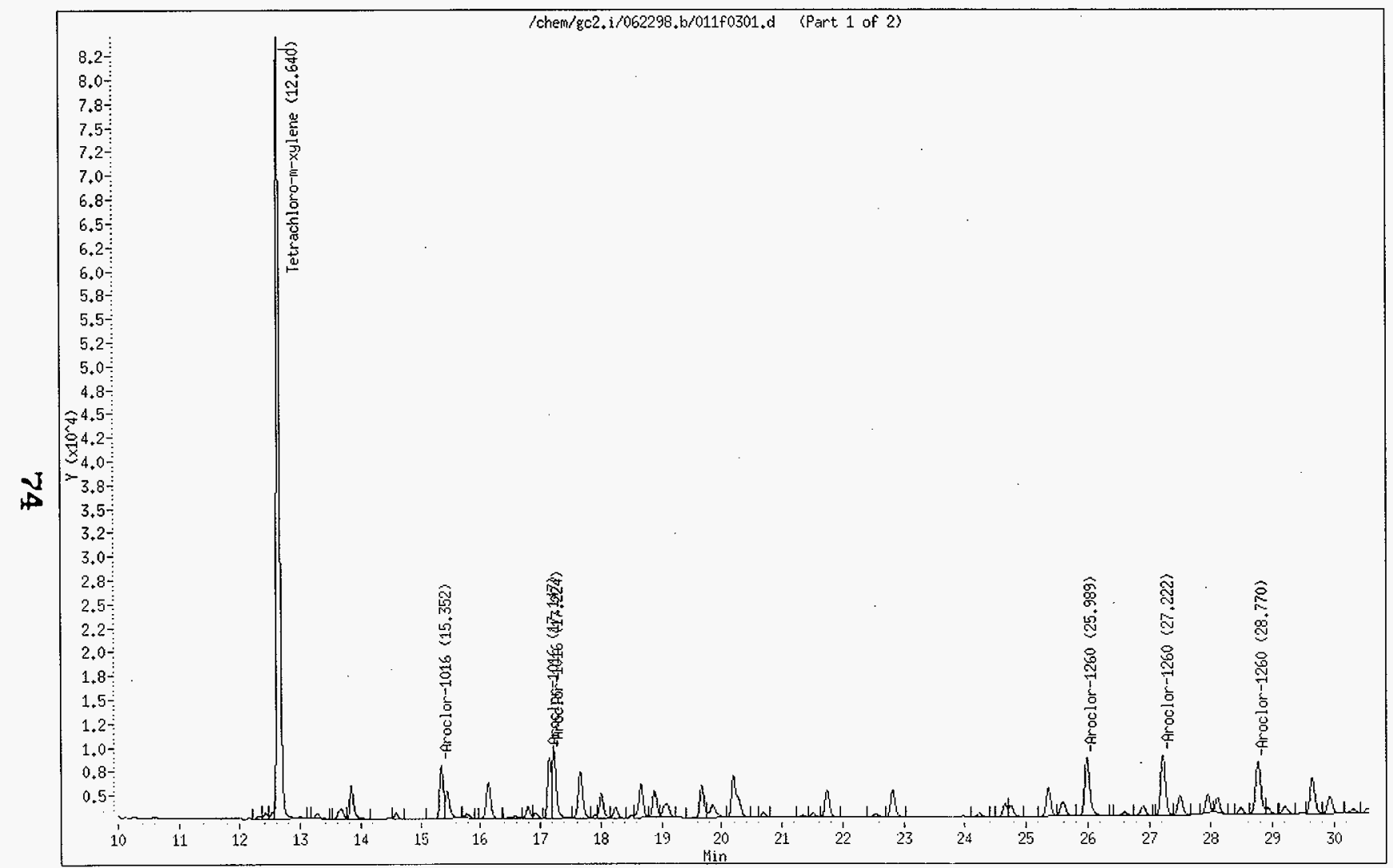


$-9823 \div 50$

Client ID: $200 \mathrm{ng} / \mathrm{ml}$ A1660

Sample Info: 200ng/ml A1660

Volume Injected $(u L): 1,0$

Column phase: Xti-5

Instrument: gc2.i

Operator: Gerald Ross

Column diameter: 0.25

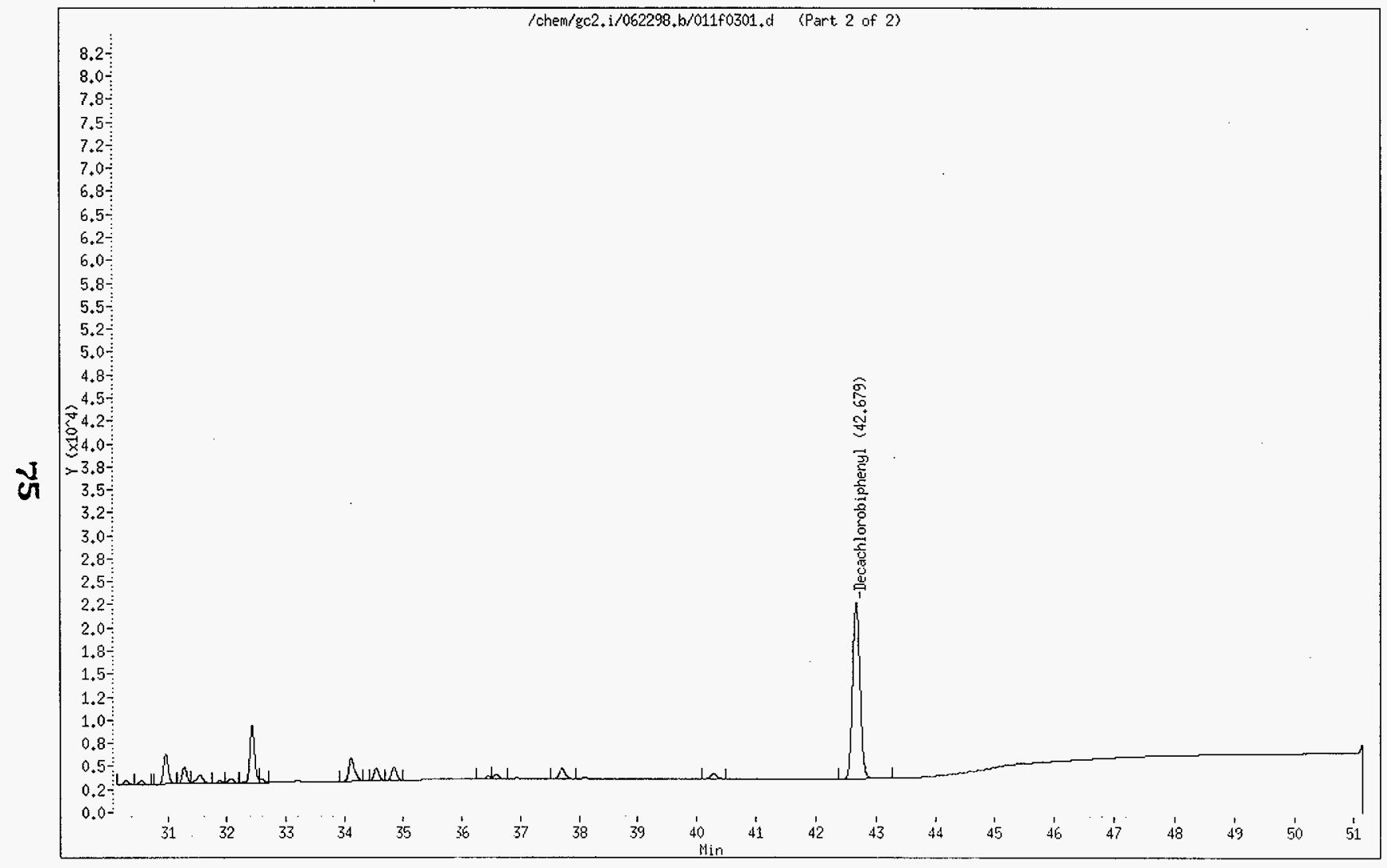


Data File: /chem/gc2,i/062298.b/012f0401.d

Page 1

Report Date: 29-Jun-19.98 13:39

\section{2-S Laboratory}

Data file:/chem/gc2.i/062298.b/012f0401.d

Lab Smp Id: $200 \mathrm{ng} / \mathrm{ml}$ A1254

Inj Date : 23-JUN-98 01:46

operator : Gerald Ross

Client Smp ID: $200 \mathrm{ng} / \mathrm{mI}$ A1254

Smp Info : $200 \mathrm{ng} / \mathrm{ml} \mathrm{A1254}$

Inst ID: $9 \mathrm{C} 2 . \mathrm{i}$

Misc Info: $200 \mathrm{ng} / \mathrm{ml} \mathrm{A} 1254$

comment

Method : /chem/gc2.i/062298.b/rcrapcb.m

Meth Date : 24-Jun-1998 09:58 gar

Cal Date : 22-MAY-1998 20:13

Als bottle: 1

Dil Factor: 1.000

Integrator: HP Genie

Target Version: 3.10

Quant Type: ESTD

Cal File: 028f0401,d

QC Sample: METHSPIKE

Compound Sublist: AR1254.sub

Sample Matrix: WATER

\begin{tabular}{|c|c|c|c|c|c|c|c|}
\hline & & & & CONCENTR & ATIONS & & \\
\hline & & & & $\mathrm{ON}-\mathrm{COL}$ & FINAL & & \\
\hline RT & EXP RT & OLT RT & RESPONSE & ( $\quad n g)$ & $(\mathrm{ug} / \mathrm{L})$ & TARGET RANGE & RATIO \\
\hline$==$ & $==-==0$ & $===x===x$ & $===== \pm==0$ & $== \pm====$ & $==== \pm==$ & 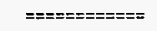 & $=$ \\
\hline $27 \mathrm{Ar}$ & roclor-12 & 254 & & & CAS \#: & : $11097-69-1$ & \\
\hline 21.740 & 21.767 & -0.027 & 180380 & 0.221 & 4.42 & & 100.00 \\
\hline 22.820 & 22.846 & -0.026 & 233311 & 0.216 & 4.33 & $112.00-152.00$ & 129.34 \\
\hline 24.754 & 24.780 & -0.026 & 222327 & 0.230 & 4.60 & $96.00-136.00$ & 123.25 \\
\hline & & Average of Peak & Concentra & ations = & 4.45 & & \\
\hline$\$ 29 \mathrm{re}$ & etrachlor & ro-m-xylene & & & CAS \#: & $877-09-8$ & \\
\hline 12.641 & 12.654 & -0.013 & 1425728 & 0.0967 & 1.93 & & 100.00 \\
\hline$\$ 30 \mathrm{DE}$ & ecachloro & obiphenyt & & & CAS $\#$ : & $2051-24-3$ & \\
\hline 42.680 & 42.728 & -0.048 & 956554 & 0.118 & 2.35 & & 100.00 \\
\hline
\end{tabular}


Data File: /chem/gc2.i/062298.b/012f0401.d

Report Date: 29-Jun-1998 13:39

$$
\begin{aligned}
& \text { 222-S Laboratory } \\
& \text { RECOVERY REPORT }
\end{aligned}
$$

Client Name:

Sample Matrix: LIQUID

Lab Smp Id: 200ng/ml A1254

Level: LoW

Data Type: GC MULTI COMP

Spikelist File: Aroclor-1254.spk

Method File:/chem/gc2.i/062298.b/rcrapcb.m

Client SDG: 061698

Fraction: PEST

Client Smp ID: 200ng/ml A1254

Operator: Gerald Ross

SampleType: METHSPIKE

Quant Type: ESTD

\begin{tabular}{|c|c|c|c|c|}
\hline SPIKE COMPOUND & $\begin{array}{l}\text { CONC } \\
\text { ADDED } \\
\mathrm{ug} / \mathrm{L}\end{array}$ & $\begin{array}{l}\text { CONC } \\
\text { RECOVERED } \\
\text { ug/L }\end{array}$ & $\begin{array}{c}\% \\
\text { RECOVERED }\end{array}$ & LIMITS \\
\hline 27 Aroclor-1254 & 4.00 & 4.45 & 111.24 & $\overline{50-150}$ \\
\hline SURROGATE COMPOUND & $\begin{array}{l}\text { CONC } \\
\text { ADDED } \\
\mathrm{ug} / \mathrm{L}\end{array}$ & $\begin{array}{l}\text { CONC } \\
\text { RECOVERED } \\
\text { ug/L }\end{array}$ & $\begin{array}{c}\% \\
\text { RECOVERED }\end{array}$ & LIMITS \\
\hline $\begin{array}{lll}\$ & 29 & \text { Tetrachloro-m-xyle } \\
\$ & 30 & \text { Decachlorobiphenyl }\end{array}$ & $\begin{array}{l}2.00 \\
2.00\end{array}$ & $\begin{array}{l}1.93 \\
2.35\end{array}$ & $\begin{array}{r}96.69 \\
117.58\end{array}$ & $\begin{array}{l}50-150 \\
50-150\end{array}$ \\
\hline
\end{tabular}

Misc Info: $200 \mathrm{ng} / \mathrm{ml}$ A1254 
Data File: /chem/gc2.i/062298.b/012f0401.d

Report Date: 29-Jun-1998 13:39

222-S Laboratory

TARGET COMPOUNDS

Client Name:

Lab Smp Id: 200ng/ml A1254

Sample Location:

Sample Date:

Sample Matrix: WATER

Analysis Type: PEST

Data Type: GC MULTI COMP

$\mathrm{Misc}$ Info: $200 \mathrm{ng} / \mathrm{ml}$ A 1254
Client SDG: 061698

Client Smp ID: $200 \mathrm{ng} / \mathrm{ml} \mathrm{A1254}$

Sample point:

Date Received:

Quant Type: ESTD

Level: LOW

operator: Gerald Ross

CAS NO.

COMPOUND

CONCENTRATION UNITS:

( $\mathrm{ug} / \mathrm{L}$ or $\mathrm{ug} / \mathrm{KG}$ ) $\mathrm{ug} / \mathrm{L}$

Q

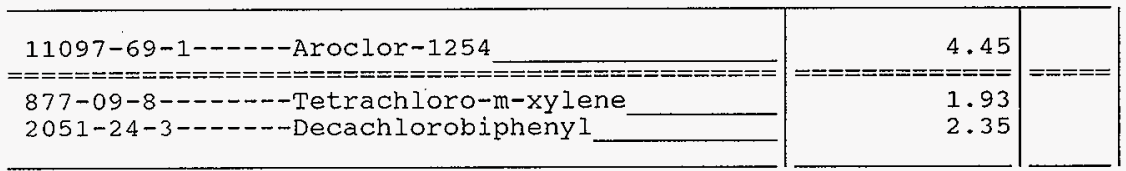


Data File: /chem/gc2, i/062298,b/012f0401.d

Date : 23-JUNA-98 01:46

Client ID: $200 \mathrm{ng} / \mathrm{ml} \mathrm{A1254}$

Sample Info: 200ng/ml A1254

Volume Injected $(u L): 1,0$

Columin phase: $\mathrm{xti}-5$

Operator: Gerald Ross

Column diameter: 0.25

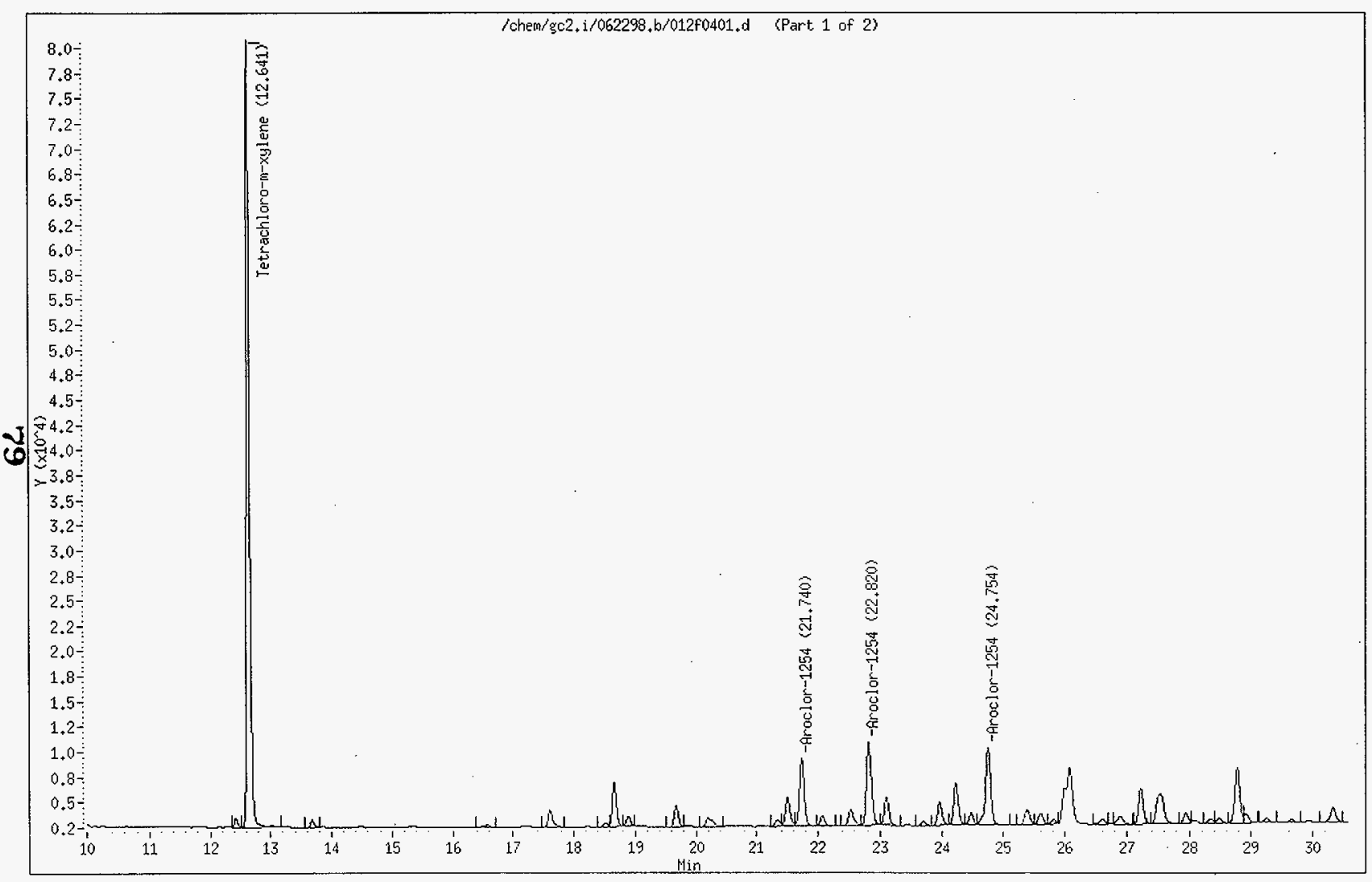


Date : 23-JuN4-98 01:46

Client ID: 200ng/ml A1254

Sample Info: 200ng/ml A1254

Yolume Injected (uL): 1,0

Column phase: Xti $\mathrm{X}-5$
Instrument: gc2, $j$

Operator: Gerald Ross

Columin diameter: 0.25

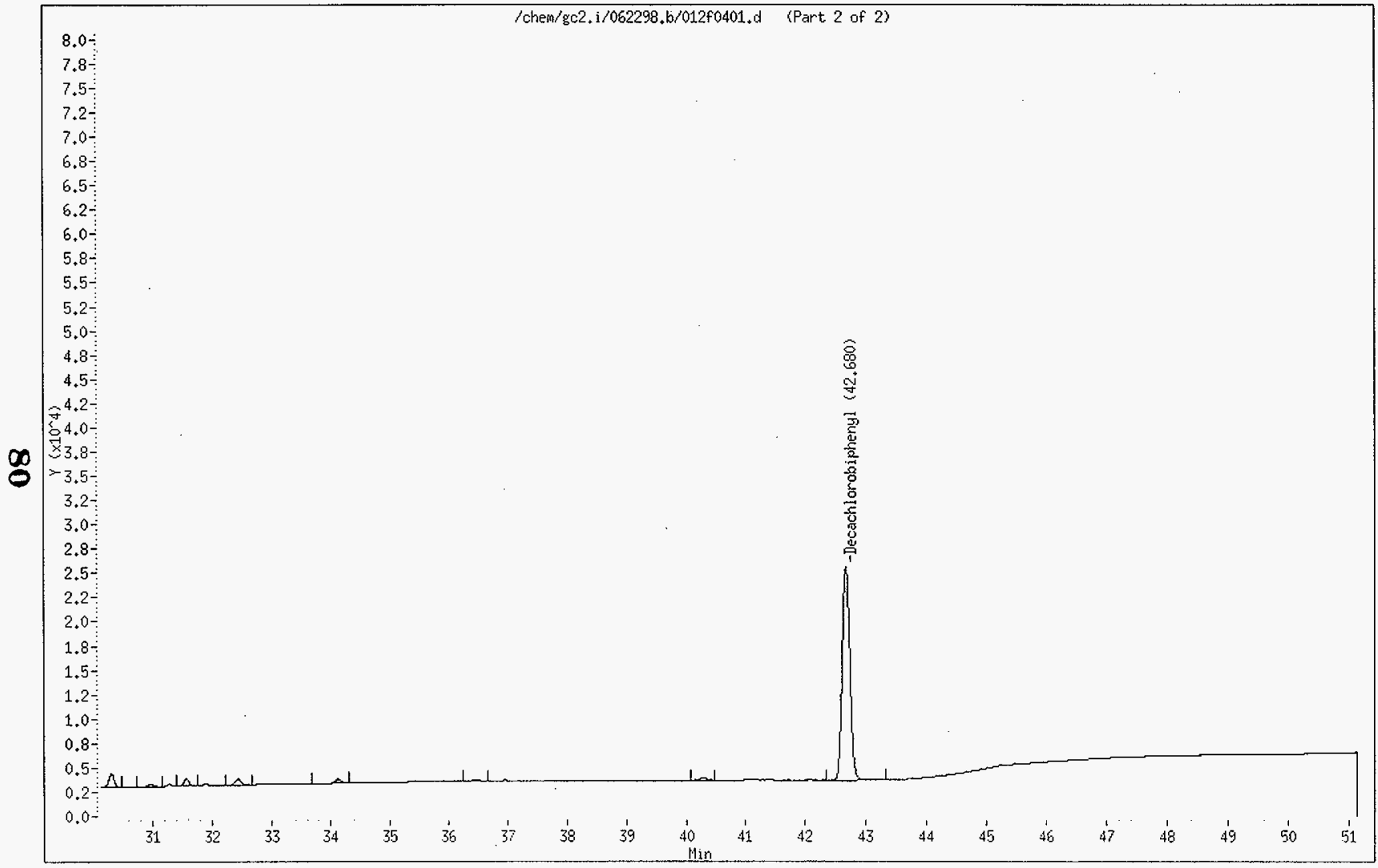




\section{2-S Laboratory}

Data file:/chem/gc2.i/062298.b/013f0401.d

Lab Smp Id: iblnk Client Smp ID: iblnk

Inj Date : 23-JUN-1998 02:44

operator : Gerald Ross Inst ID: gc2.i

Smp Info : iblnk

Misc Info: iblnk

Comment :

Method : /chem/gc2.i/062298.b/rcrapcb.m

Meth Date : 17-Jul-1998 09:20 gar Quant Type: ESTD

Cal Date : 22-JUN-98 19:59 Cal File: 007f0301.d

Als bottle: 1

Dil Factor: 1.000

Integrator: HP Genie

Target Version: 3.10

Compound Sublist: Aroclors.sub Sample Matrix: WATER

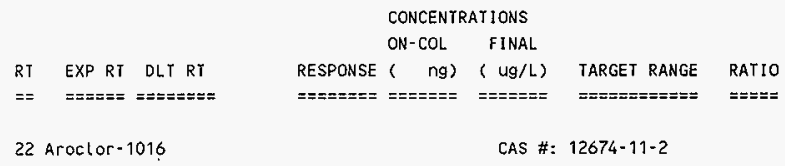

Peaks not detected for Quant. or Qual. signal(s).

23 Aroclor -1221

CAS \#: $11104-28-2$

Peaks not detected for Quant. or Qual. signal(s).

24 aroclor -1232 CAS \#: $1114-16-5$

Peaks not detected for Quant. or Qual. signal(s).

25 Aroclor -1242

CAS \#: $53469-21-9$

Peaks not detected for Quant. or Qual. signal(s).

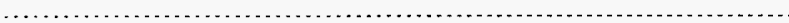

26 Aroclor -1248

CAS \#: $12672-29-6$

Peaks not detected for Quant. or Qual. signal(s).

27 aroclor $1254 \quad$ CAS \#: $11097-69-1$

Peaks not detected for Quant. or Qual. signal(s). 
Data File: /chem/gc2.i/062298.b/013f0401.d Report Date: 20-Jul-1998 07:56

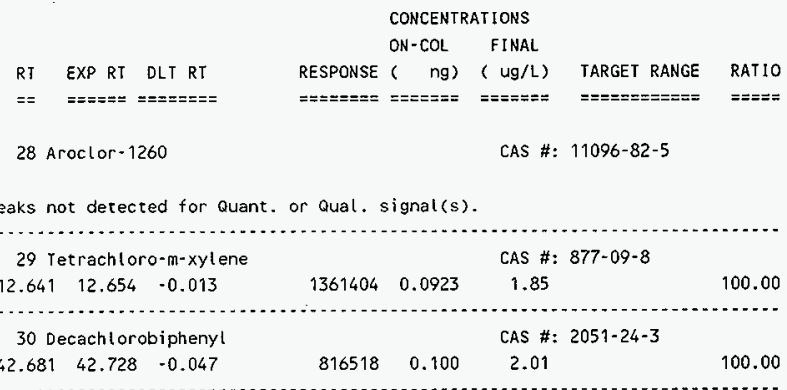


Data File: /chem/gc2.i/062298.b/013f0401.d

Report Date: 20-Jul-1998 07:56

222-S Laboratory

RECOVERY REPORT

client Name:

Client SDG: 062298

Sample Matrix: LIQUID

Fraction: PEST

Lab Smp Id: iblnk

Client Smp ID: iblnk

Level: LoW

Data Type: GC MULT COMP

Spikelist File: RESOLUTION.spk

Method File:/chem/gc2.i/062298.b/rcrapcb.m

Operator: Gerald Ross

SampleType: SAMPLE

Quant Type: ESTD

Misc Info: iblnk

\begin{tabular}{|c|c|c|c|c|}
\hline SURROGATE COMPOUND & $\begin{array}{l}\text { CONC } \\
\text { ADDED } \\
u g / L\end{array}$ & $\begin{array}{c}\text { CONC } \\
\text { RECOVERED } \\
u g / L\end{array}$ & $\stackrel{\circ}{\%}$ RECOVERED & LIMITS \\
\hline $\begin{array}{l}\$ 29 \text { Tetrachloro-m-xyle } \\
\$ 30 \text { Decachlorobiphenyl }\end{array}$ & $\begin{array}{l}2.00 \\
2.00\end{array}$ & $\begin{array}{l}1.85 \\
2.01\end{array}$ & $\begin{array}{r}92.33 \\
100.36\end{array}$ & $\begin{array}{l}50-150 \\
50-150\end{array}$ \\
\hline
\end{tabular}


Data File: /chem/gc2.i/062298.b/013f0401.d

\section{2-s Laboratory \\ TARGET COMPOUNDS}

client Name:

Lab Smp Id: iblnk

Sample Location:

Sample Date:

Sample Matrix: WATER

Analysis Type: PEST

Data Type: GC MULTI COMP

Misc Info: iblnk
Client SDG: 062298

Client Smp ID: iblnk

Sample Point:

Date Received:

Quant Type: ESTD

Level: LOW

Operator: Gerald Ross

CAS NO.

COMPOUND

CONCENTRATION UNITS:

( $\mathrm{ug} / \mathrm{L}$ or $\mathrm{ug} / \mathrm{KG}) \mathrm{ug} / \mathrm{L}$

12674-11-2----Aroclor -1016

11104-28-2-----Aroclor-1221

$1114-16-5-\cdots---A r o c l o r-1232$

$53469-21-9------$ Aroclor -1242

12672-29-6-----Aroclor -1248

1.1097-69-1-----Aroclor -1254

11096-82-5------Aroclor-1260

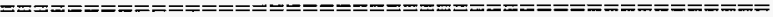

877-09-8-------Tetrachloro-m-xylene

2051-24-3-----Decach lorobiphenyl

\begin{tabular}{|c|c|}
0.600 & $\mathrm{U}$ \\
0.600 & $\mathrm{U}$ \\
0.600 & $\mathrm{U}$ \\
0.600 & $\mathrm{U}$ \\
0.600 & $\mathrm{U}$ \\
0.600 & $\mathrm{U}$ \\
0.600 & $\mathrm{U}$ \\
$==========$ & $====$ \\
1.85 &. \\
2.01 & \\
\end{tabular}


Date : 23-JUN-1998 02:44

Client ID: iblnk

Sample Info: iblnk

Volume Injected (uL): 1.0

Column phase: $x$ ti-5

Instrument: gc2, 1

Operator: Gerald Ross

Column diameter: 0.25

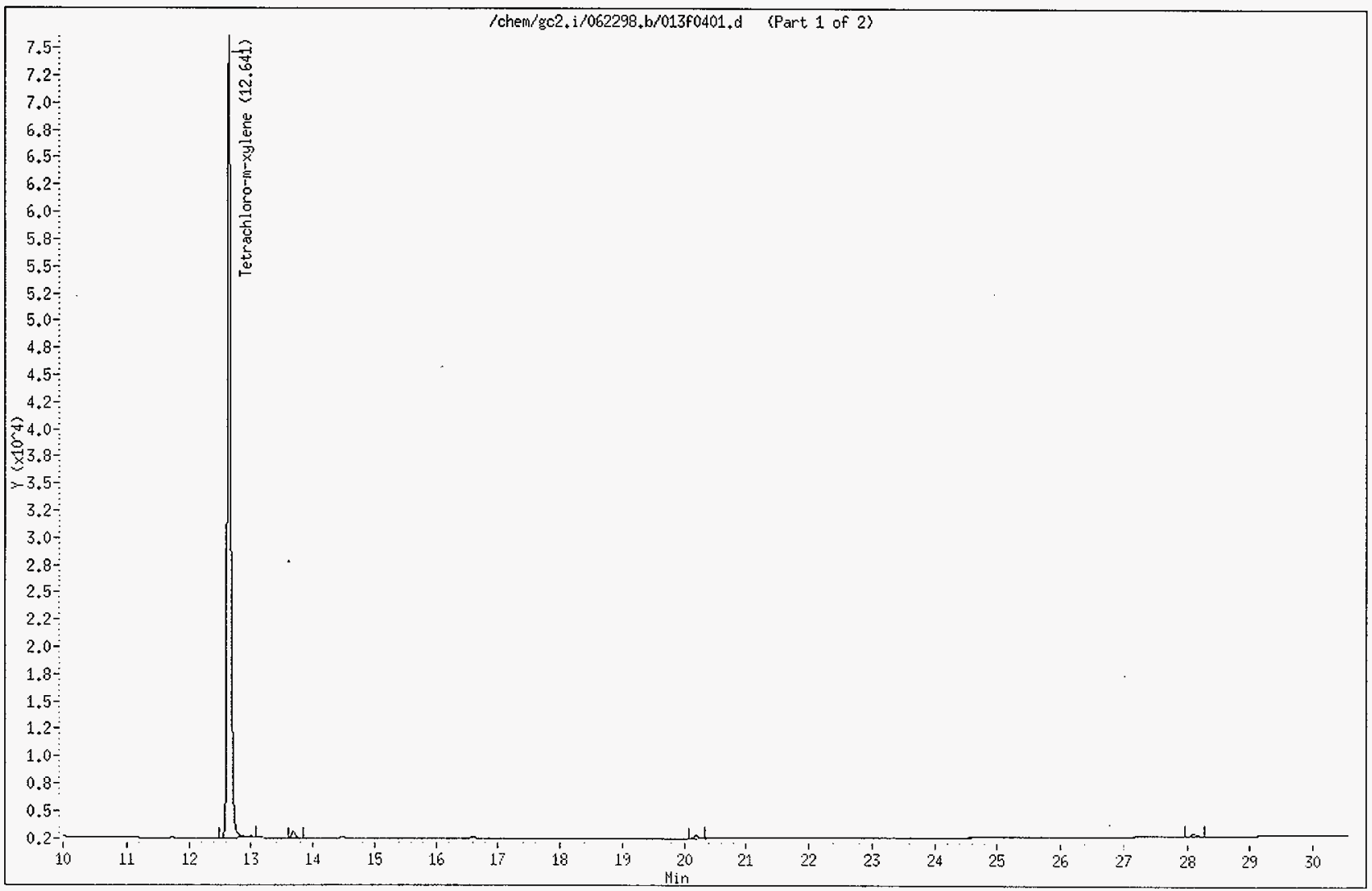


Data File: /chem/gc2,i/062298.b/013f0401.d

Date : 23-JuN-1998 02:44

Client. II: iblnk

Sample Info: iblnk

Volume Injected (uL): 1.0

Column phase: Xti-5

Instrument: $\mathrm{gc} 2 . \mathrm{i}$

Operator: Gerald Ross

Column diameter: 0.25

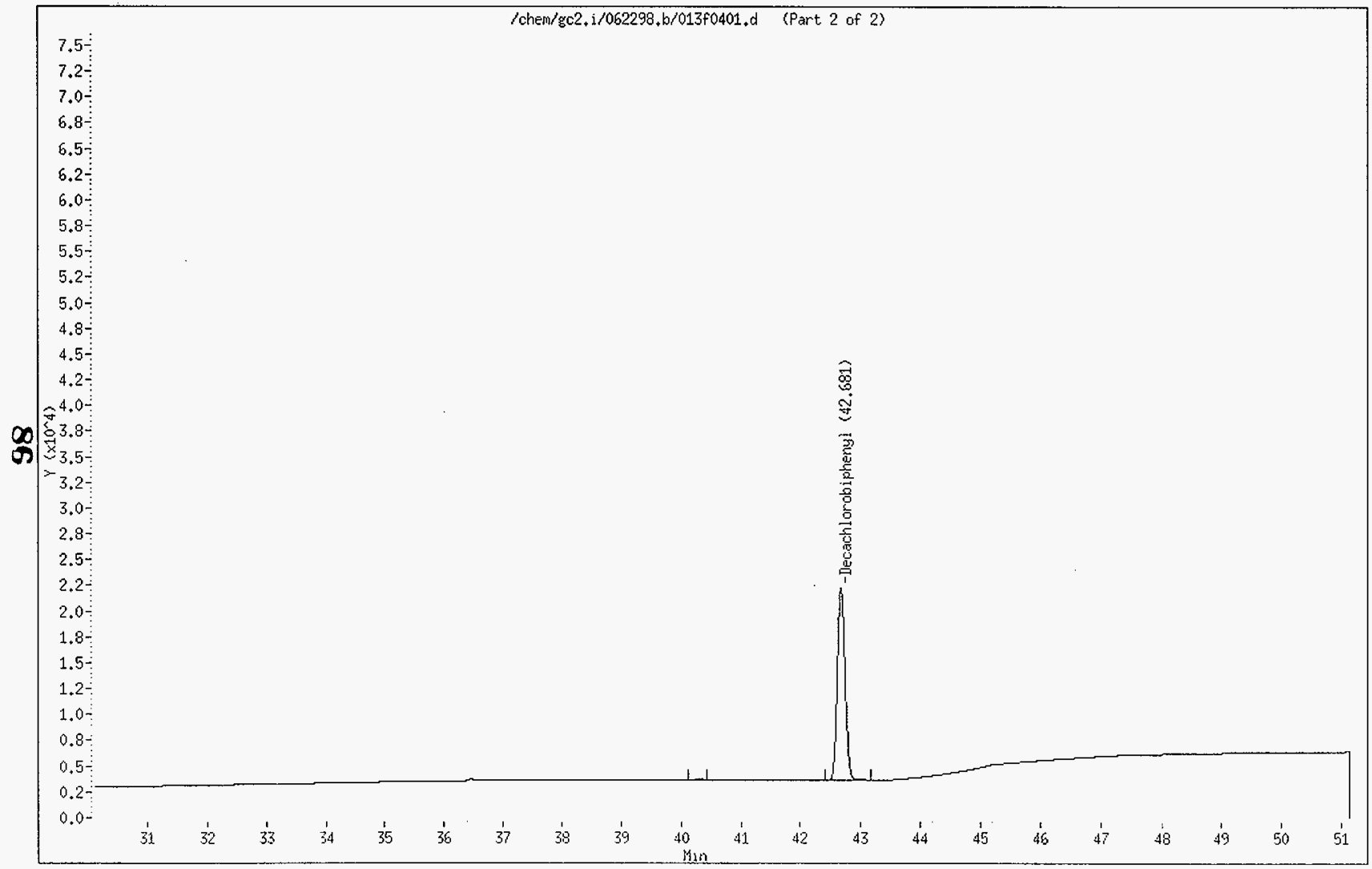

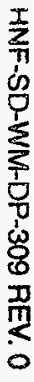


Data File: /chem/gc2.i/062298.b/014f0401.d

Report Date: 20-Jul-1998 07:56

Data file:/chem/gc2.i/062298.b/014f0401.d

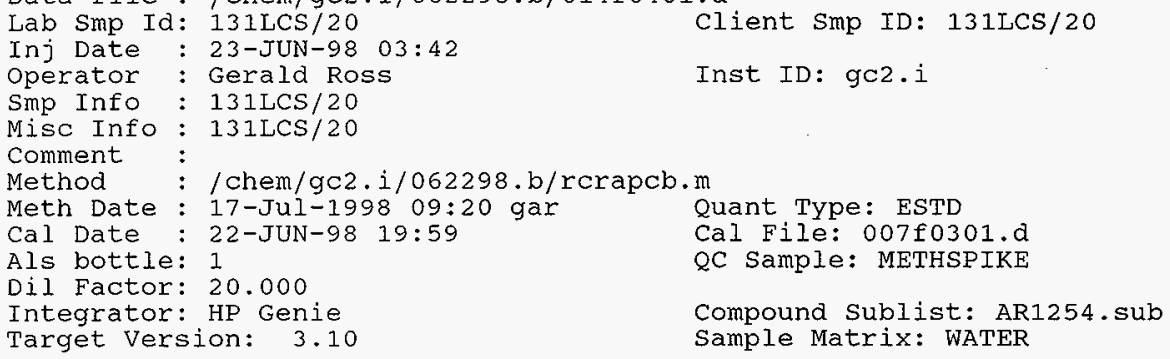

CONCENTRATIONS

ON-COL FINAL

RT EXP RT DLT RT RESPONSE ( ng) ( $\mathrm{Ug} / \mathrm{L}$ ) TARGET RANGE RATIO

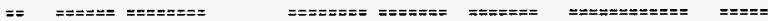

27 Aroclor -1254

CAS \#: $11097-69-1$

$\begin{array}{llll}21.741 & 21.767 & -0.026\end{array}$

$\begin{array}{lll}143378 & 0.176 & 70.4\end{array}$

100.00

$22.822 \quad 22.846 \quad-0.024$

$187168 \quad 0.174$

$69.4112 .00-152.00$

130.54

$24.753 \quad 24.780 \quad-0.027$

$174507 \quad 0.180$

$72.196 .00-136.00$

121.71

Average of Peak Concentrations $=70.6$

$\$ 29$ Tetrachioro-m-xylene

CAS \#: $877-09-8$

$12.642 \quad 12.654 \quad-0.012$

$1030912 \quad 0.0699$

28.0

100.00

$\$ 30$ Decachlorobiphenyt

CAS \#: $2051 \cdot 24 \cdot 3$

$\begin{array}{llll}42.682 & 42.728 & -0.046\end{array}$

$788626 \quad 0.0969$

38.8

100.00 
Data File: /chem/gc2.i/062298.b/014f0401.d

Report Date: 20-Jul-1998 07:56

\section{2-S Laboratory}

RECOVERY REPORT

Client Name:

Sample Matrix: LIQUID

Client SDG: 062298

Iab Smp Id: 131LCS/20

Fraction: PEST

Level: LoW

Client Smp ID: 131LCS/20

Data Type: GC MULTI COMP

SpikeList File: Aroclor-1254.spk

Method File: / chem/gc2.i/062298.b/rcrapcb.m

Operator: Gerald Ross

SampleType: METHSPIKE

Quant Type: ESTD

Misc Info: $131 \mathrm{LCS} / 20$

\begin{tabular}{|c|c|c|c|c|}
\hline SPIKE COMPOUND & $\begin{array}{l}\text { CONC } \\
\text { ADDED } \\
\mathrm{ug} / \mathrm{L}\end{array}$ & $\begin{array}{c}\text { CONC } \\
\text { RECOVERED } \\
\mathrm{ug} / \mathrm{L}\end{array}$ & $\stackrel{\circ}{\%}$ RECOVERED & IIMITS \\
\hline 27 Aroclor-1254 & 80.0 & 70.6 & 88.30 & $\overline{50-150}$ \\
\hline SURROGATE COMPOUND & $\begin{array}{l}\text { CONC } \\
\text { ADDED } \\
\text { ug/L }\end{array}$ & $\begin{array}{c}\text { CONC } \\
\text { RECOVERED } \\
u g / L\end{array}$ & RECOVERED & LIMITS \\
\hline $\begin{array}{lll}\$ & 29 & \text { Tetrachloro-m-xyle } \\
\$ & 30 \text { Decachlorobiphenyl }\end{array}$ & $\begin{array}{l}40.0 \\
40.0\end{array}$ & $\begin{array}{l}28.0 \\
38.8\end{array}$ & $\begin{array}{l}69.91 \\
96.94\end{array}$ & $\begin{array}{l}50-150 \\
50-150\end{array}$ \\
\hline
\end{tabular}




\section{HNF-SD-WM-DP-309 REV. 0}

Data File: /chem/gc2.i/062298.b/014f0401.d

Report Date: 20-Jul-1998 07:56

$$
\begin{aligned}
& \text { 222-S Laboratory } \\
& \text { TARGET COMPOUNDS }
\end{aligned}
$$

Client Name:

Lab Smp Id: $131 \mathrm{LCS} / 20$

Sample Location:

Sample Date:

Sample Matrix: WATER

Analysis Type: PEST

Data Type: GC MULTI COMP

Misc Info: 131LCS/20
Client SDG: 062298

Client Smp ID: 131LCS/20

Sample point:

Date Received:

Quant Type: ESTD

Level: LoW

Operator: Gerald Ross

CONCENTRATION UNITS :

(ug/L or $\mathrm{ug} / \mathrm{KG}$ ) ug/L

\begin{tabular}{|c|c|c|}
\hline $11097-69-1-\cdots--$ Aroclor -1254 & 70.6 & \\
\hline 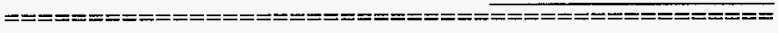 & 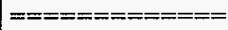 & $====$ \\
\hline $\begin{array}{l}877-09-8------ \text { Tetrachloro-m-xylene } \\
2051-24-3------ \text { Decachlorobiphenyl }\end{array}$ & $\begin{array}{l}28.0 \\
38.8\end{array}$ & \\
\hline
\end{tabular}




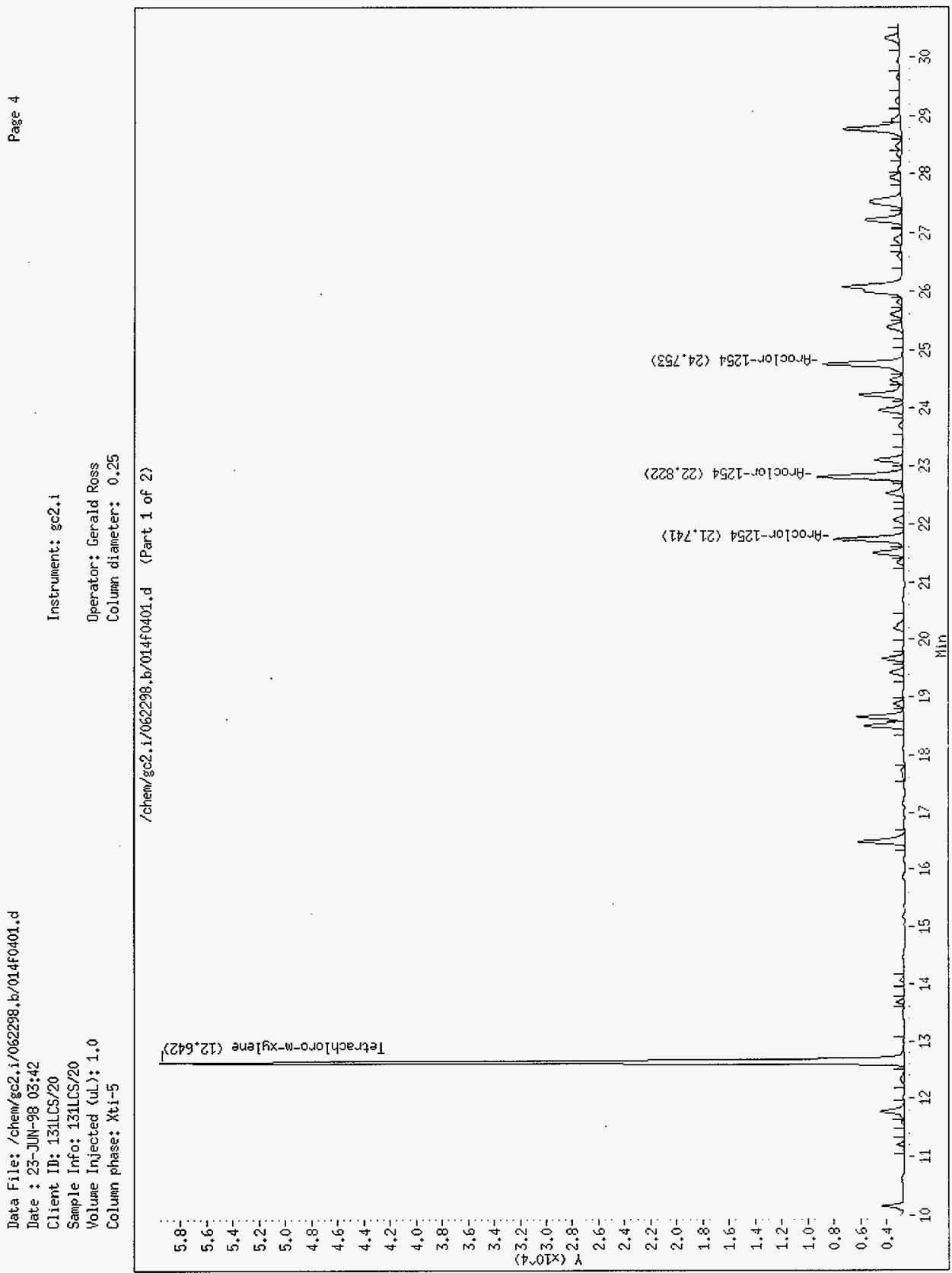


Data File: /chem/gc2, i/062298,b/014f0401,d

Date * 23-JUN-98 03:42

Client ID: 131LCS/20

Instrument: gc2. $\mathrm{i}$

Sample info: 131LCS/20

Volume Injected (uL) $: 1,0$

Operator: Gerald Ross

Column phase: Xti-5

Column diameter: 0.25

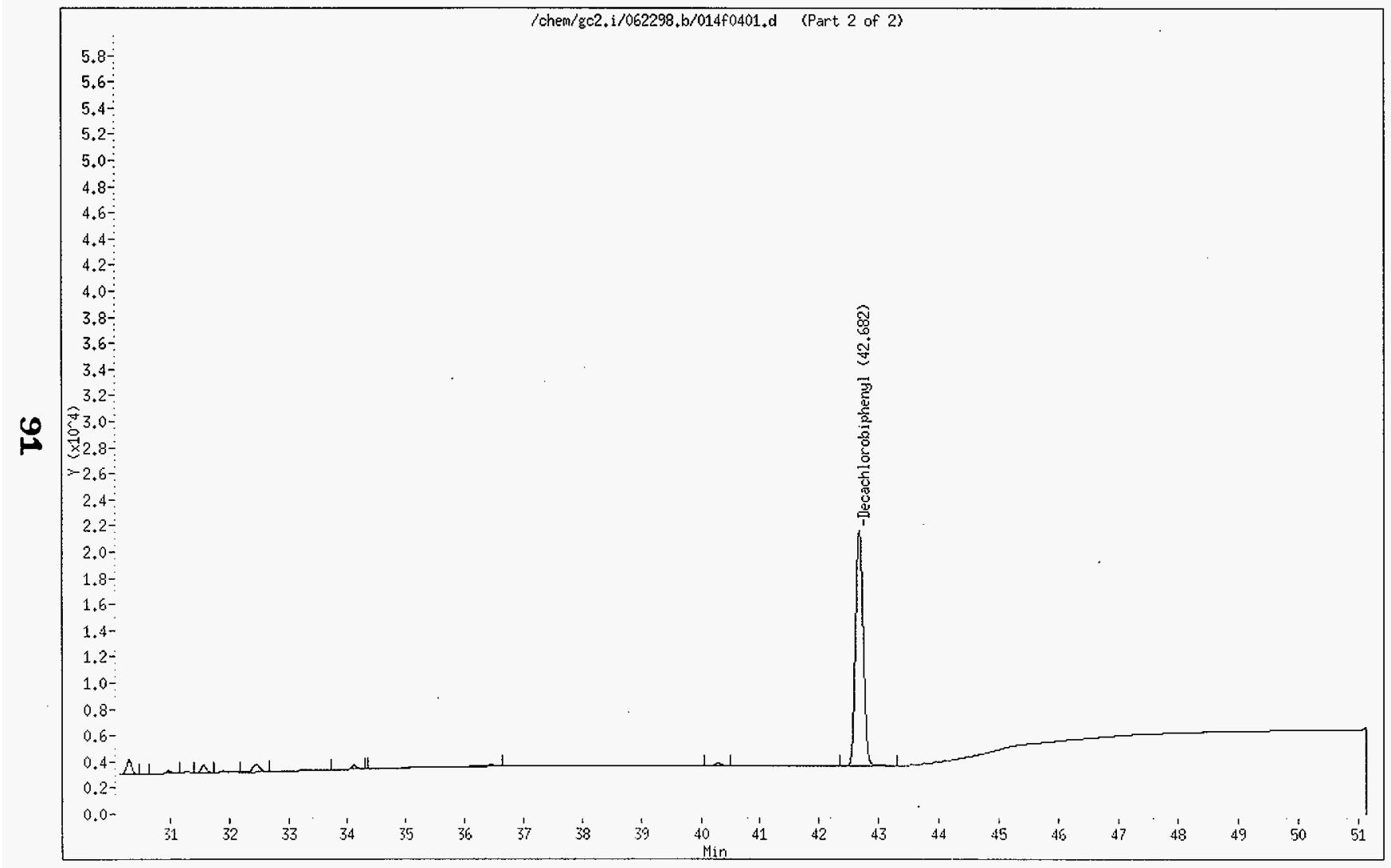


Data File: /chem/gc2.i/062298.b/015f0401.d

Report Date: 20-Jul-1998 07:35

\section{2-S Laboratory}

Data file : /chem/gc2.i/062298.b/015f0401.d

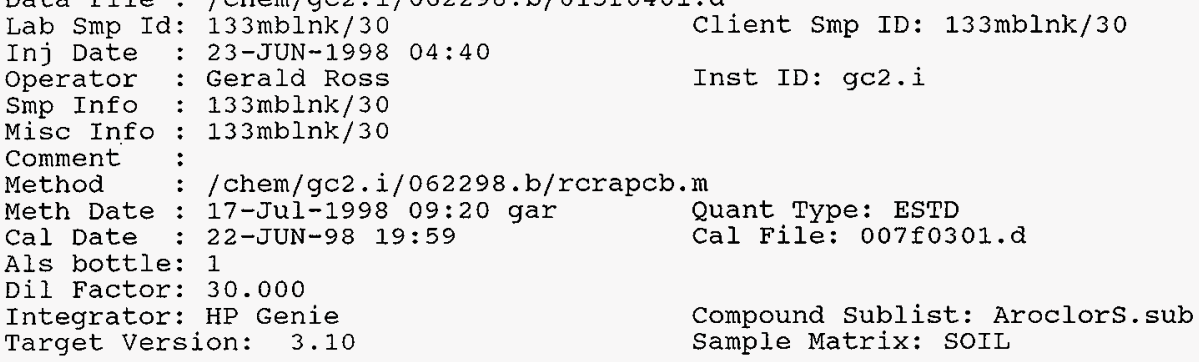

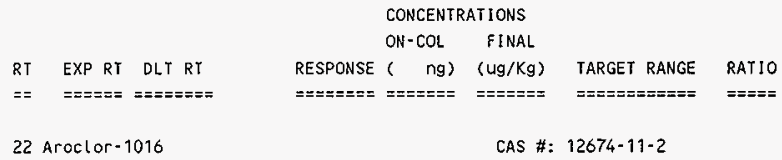

Peaks not detected for Quant. or Qual. signal(s).

23 Aroclor -1221

CAS \#: $11104-28-2$

Peaks not detected for Quant. or Quat. signal(s).

24 Aroclor -1232

CAS \#: $1114-16-5$

Peaks not detected for Quant. or Qual, signal $(\$)$.

25 Aroclor -1242

CAS \#: $53469-21-9$

Peaks not detected for Quant, or Qual, signal(s).

26 Aroclor -1248

CAS \#: $12672-29-6$

Peaks not detected for Quant. or Qual. signal(s).

27 Aroclor -1254

CAS \#: 11097-69-1

Peaks not detected for Quant. or Qual. signal(s). 
Data File:/chem/gc2.i/062298.b/015f0401.d

Report Date: 20-Jul-1998 07:35

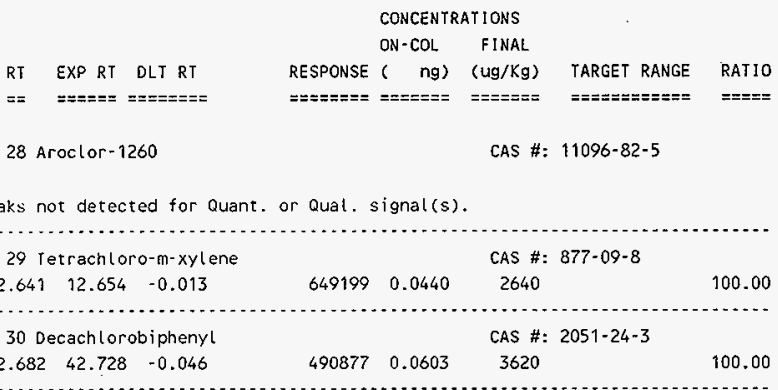




\section{2-S Laboratory}

\section{RECOVERY REPORT}

Client Name:

Sample Matrix: SOLID

Lab Smp Id: $133 \mathrm{mblnk} / 30$

Level: LOW

Data Type: GC MULTI COMP

Spikelist File: RESOLUTION.spk

Method File: /chem/gc2.i/062298.

Misc Info: $133 \mathrm{mblnk} / 30$
Client SDG: 062298

Fraction: PEST

Client Smp ID: $133 \mathrm{mblnk} / 30$

operator: Gerald Ross

SampleType: SAMPLE

Quant Type: ESTD

\begin{tabular}{|c|c|c|c|c|}
\hline SURROGATE COMPOUND & $\begin{array}{l}\text { CONC } \\
\text { ADDED }\end{array}$ & $\begin{array}{c}\text { CONC } \\
\text { RECOVERED }\end{array}$ & $\begin{array}{c}\% \\
\text { RECOVERED }\end{array}$ & LIMITS \\
\hline $\begin{array}{lll}\$ & 29 & \text { Tetrachloro-m-xyle } \\
\$ & 30 & \text { Decachlorobiphenyl }\end{array}$ & $\begin{array}{l}4000 \\
4000\end{array}$ & $\begin{array}{l}2640 \\
3620\end{array}$ & $\begin{array}{l}66.04 \\
90.51\end{array}$ & $\begin{array}{l}50-150 \\
50-150\end{array}$ \\
\hline
\end{tabular}


Data File:/chem/gc2.i/062298.b/015f0401.d Report Date: 20-Jul-1998 07:35

\section{2-S Laboratory \\ TARGET COMPOUNDS}

Client Name:

Lab Smp Id: 133mblnk/30

Sample Location:

Sample Date:

Sample Matrix: Sort

Analysis Type: PEST

Data Type: GC MULTI COMP

Misc Info: $133 \mathrm{mblnk} / 30$
Client SDG: 062298

Client Smp ID: $133 \mathrm{mblnk} / 30$

Sample Point:

Date Received:

quant Type: ESTD

Level: IOW

operator: Gerald Ross

CONCENTRATION UNITS:

CAS NO.

COMPOUND

( $\mathrm{ug} / \mathrm{L}$ or $\mathrm{ug} / \mathrm{KG}$ ) $\mathrm{ug} / \mathrm{Kg}$

\begin{tabular}{r|c}
990 & $\mathrm{U}$ \\
990 & $\mathrm{U}$ \\
990 & $\mathrm{U}$ \\
990 & $\mathrm{U}$ \\
990 & $\mathrm{U}$ \\
990 & $\mathrm{U}$ \\
990 & $\mathrm{U}$ \\
$=\mathrm{m}=========$ & $===$ \\
2640 \\
3620
\end{tabular}


Data File: /chem/gc2, i/062298.b/015f0401,d

Date : 23-JuN-1998 04:40

Client ID: $133 \mathrm{mblnk} / 30$

Sample Info: 133 mblnk $/ 30$

Volume Injected $\{u L\rangle: 1.0$

Columin phase: Xti-5

Operator: Gerald Ross

Column diameter: 0.25

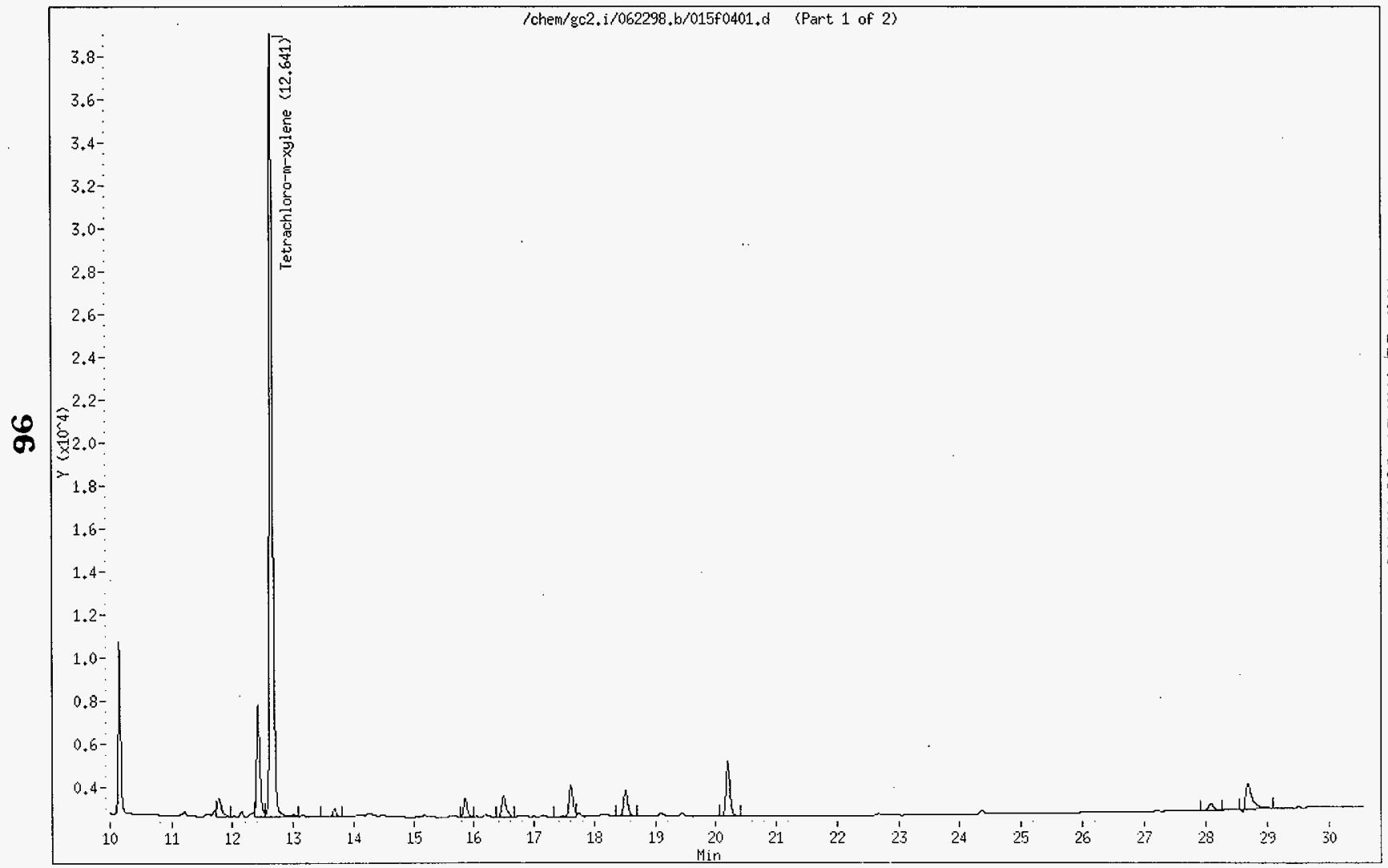


Client ID: $133 \mathrm{mblnk} / 30$

Sample Info: $133 \mathrm{mblnk} / 30$

Yolume Injected $\{\mathrm{uL}$ ): 1,0

Column phase: Xti-5
Instrument: $\mathrm{gc2}+\mathrm{i}$

Qperator: Gerald Ross

Columin diameter: 0.25

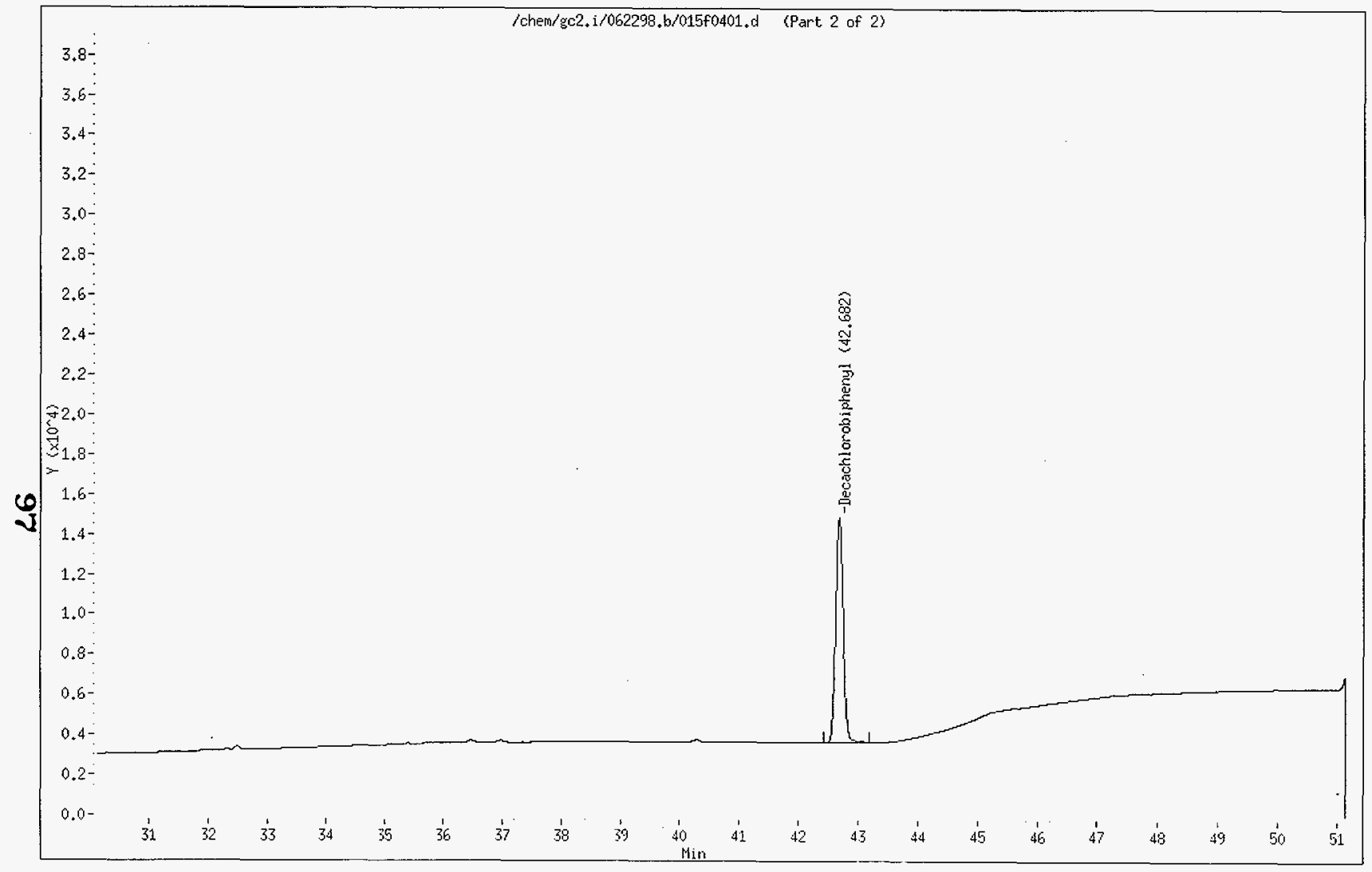


Report Date: 20-Jul-1998 06:25

\section{2-S Laboratory}

Data file : /chem/gc2.i/062298.b/018f0801.d

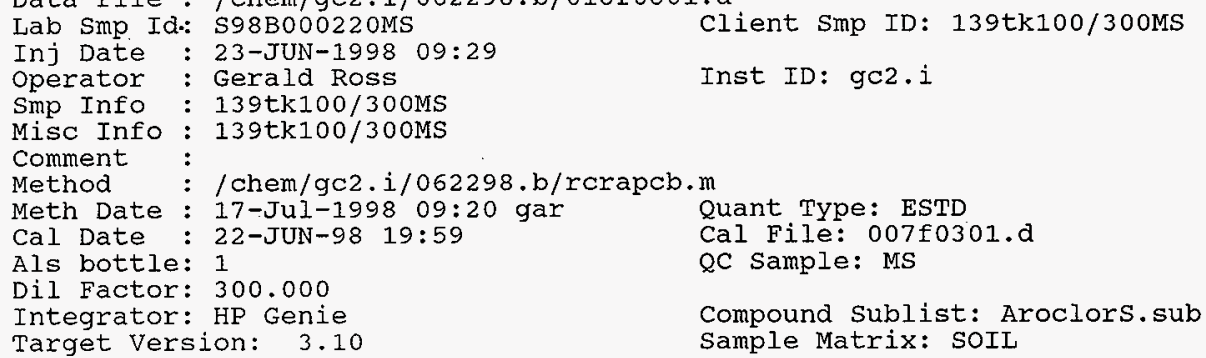

\begin{tabular}{|c|c|c|c|c|c|c|}
\hline & & & CONCENT & ATIONS & & \\
\hline & & & $\mathrm{ON}-\mathrm{COL}$ & FINAL & & \\
\hline RI & EXP RT DLT RT & RESPONSE & ( $n g$ ) & $(u g / K g)$ & TARGET RANGE & RATIO \\
\hline$==$ & 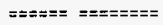 & $==\approx=\Xi=\Omega=$ & $====\Xi==$ & $==ニ= \pm=E$ & 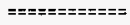 & $====+$ \\
\hline & octor-1016 & & & CAS \# & $12674-11-2$ & \\
\hline
\end{tabular}

Peaks not detected for Quant. or Qual. signal(s).

\begin{tabular}{|c|c|c|c|c|c|c|c|c|}
\hline \multicolumn{4}{|c|}{23 Aroclor-1221 } & \multicolumn{5}{|c|}{ CAS \#: $11104-28-2$} \\
\hline 10.806 & 10.811 & -0.005 & 49407 & & & & & 100.00 \\
\hline 11.920 & 11.923 & -0.003 & 6818 & & & $75.00-$ & 115.00 & 13.80 \\
\hline 13.289 & 13.282 & 0.007 & 11077 & 0.0563 & 19300 & $208.00-$ & 258.00 & 22.42 \\
\hline 13.640 & 13.673 & -0.033 & 9515 & 0.0422 & 14500 & 0.00 & 0.00 & 19.26 \\
\hline 13.851 & 13.849 & 0.002 & 35869 & 0.0719 & 24600 & 0.00 & 0.00 & 72.60 \\
\hline \multicolumn{5}{|c|}{ Average of Peak } & \multicolumn{4}{|l|}{19500} \\
\hline
\end{tabular}

Peaks not detected for Quant. or Qual. signal(s).

25 Aroclor-1242 CAS \#: 53469-21-9

Peaks not detected for Quant. or Qual. signal(s).

26 Aroclor -1248

CAS \#: $12672-29-6$

Operator disabled compound identification.

\begin{tabular}{|c|c|c|c|c|c|c|c|c|}
\hline \multicolumn{5}{|c|}{27 Aroclor-1254 } & \multicolumn{4}{|c|}{ CAS \#: $11097-69-1$} \\
\hline 21.747 & 21.767 & -0.020 & 29945 & 0.0367 & 12600 & & & 100.00 \\
\hline 22.828 & 22.846 & -0.018 & 42063 & 0.0390 & 13400 & $112.00=$ & 152.00 & 140.47 \\
\hline 24.760 & 24.780 & -0.020 & 37937 & 0.0392 & 13400 & $96.00-$ & 136.00 & 126.69 \\
\hline \multicolumn{5}{|c|}{ Average of } & 13100 & & & \\
\hline
\end{tabular}


Data File: /chem/gc2.i/062298.b/018f0801.d

Report Date: 20-Jul-1998 06:25

\begin{tabular}{|c|c|c|c|c|c|c|}
\hline & & & CONCENTR & ATIONS & & \\
\hline & & & $\mathrm{ON}-\mathrm{COL}$ & FINAL & & \\
\hline RT & EXP RT DLT RT & RESPONSE & ( $n g)$ & $(u g / K g)$ & TARGET RANGE & RATIO \\
\hline$==$ & 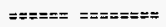 & 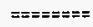 & $== \pm== \pm=$ & $====ニ==$ & $==== \pm=== \pm===$ & $=====$ \\
\hline 28 & clor-1260 & & & CAS \# & $11096-82-5$ & \\
\hline
\end{tabular}

Peaks not detected for Quant. or Quat. signal(s).

$\$ 29$ retrachloro-m-xylene

CAS \#: $877-09-8$

$12.648 \quad 12.654 \quad-0.006$

$92790 \quad 0.00629$

2160

100.00

$\$ 30$ Decachlorobiphenyl

CAS \#: $2051-24-3$

$42.684 \quad 42.728 \quad-0.044$

$99132 \quad 0.0122$

4180

$100.00(R)$

QC Flag Legend

R - Spike/Surrogate failed recovery limits. 
Data File: /chem/gc2.i/062298.b/018f0801.d

Report Date: 20-Jul-1998 06:25

\section{2-S Laboratory \\ RECOVERY REPORT}

client Name:

Sample Matrix: SOLID

Client SDG: 062298

Lab Smp Id: S98B000220MS

Fraction: PEST

Level: LOW

Data Type: GC MULTI COMP

Spikelist File: Aroclor-1254.spk

Method File: /chem/gc2.i/062298.b/rcrapcb.m

Misc Info: 139tk100/300MS

\begin{tabular}{|c|c|c|c|c|}
\hline SPIKE COMPOUND & $\begin{array}{l}\text { CONC } \\
\text { ADDED } \\
\mathrm{ug} / \mathrm{Kg}\end{array}$ & $\begin{array}{c}\text { CONC } \\
\text { RECOVERED } \\
\text { ug/Kg }\end{array}$ & RECOVERED & LIMITS \\
\hline 27 Aroclor -1254 & 9140 & $\begin{array}{r}13100 \\
-\quad 8430 \\
\end{array}$ & 743.69 & $50-150$ \\
\hline \multicolumn{5}{|c|}{$100 \times 4670 / 9140=51.095$} \\
\hline SURROGATE COMPOUND & $\begin{array}{l}\text { CONC } \\
\text { ADDED } \\
\mathrm{ug} / \mathrm{Kg}\end{array}$ & $\begin{array}{c}\text { CONC } \\
\text { RECOVERED } \\
\text { ug/Kg }\end{array}$ & RECOVERED & LIMITS \\
\hline $\begin{array}{l}\$ 29 \text { Tetrachloro-m-xyle } \\
\$ 30 \text { Decachlorobiphenyl }\end{array}$ & $\begin{array}{l}2280 \\
2280\end{array}$ & $\begin{array}{l}2160 \\
4180\end{array}$ & $\begin{array}{c}94.39 \\
182.78 *\end{array}$ & $\begin{array}{l}\overline{50-150} \\
50-150\end{array}$ \\
\hline
\end{tabular}


Data File: /chem/gc2.i/062298.b/018f0801.d Report Date: 20-Jul-1998 06:25

$$
\begin{aligned}
& \text { 222-S Laboratory } \\
& \text { TARGET COMPOUNDS }
\end{aligned}
$$

Client Name:

Lab Smp Id: S98B000220MS

Sample Location:

Sample Date:

Sample Matrix: SOIL

Analysis Type: PEST

Data Type: GC MULTI COMP

Misc Info: 139tk100/300MS client SDG: 062298

Client Smp ID: 139tk100/300MS

Sample Point:

Date Received:

Quant Type: ESTD

Level: LOW

Operator: Gerald Ross

CONCENTRATION UNITS:

( $u g / L$ or $u g / K G) ~ u g / K g$

\begin{tabular}{|c|c|c|}
\hline $12674-11-2-----$ Aroclor -1016 & 9900 & $\mathbf{U}$ \\
\hline $11104-28-2-----$ Aroclor -1221 & 19500 & \\
\hline $1114-16-5------$ Aroclor -1232 & 9900 & $\mathrm{U}$ \\
\hline $53469-21-9-----$ Aroclor -1242 & 9900 & $\mathrm{U}$ \\
\hline $12672-29-6-\cdots-$ Aroclor -1248 & 9900 & $\mathbf{U}$ \\
\hline $11097-69-1-\cdots--$ Aroclor -1254 & 13100 & \\
\hline $11096-82-5----$ Aroclor -1260 & 9900 & $\mathrm{U}$ \\
\hline 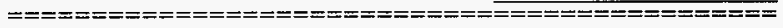 & $==========$ & $====$ \\
\hline 877-09-8--------Tetrachloro-m-xylene & 2160 & \\
\hline $2051-24-3------D e c a c h$ lorobiphenyl & 4180 & \\
\hline
\end{tabular}

$Q$ 


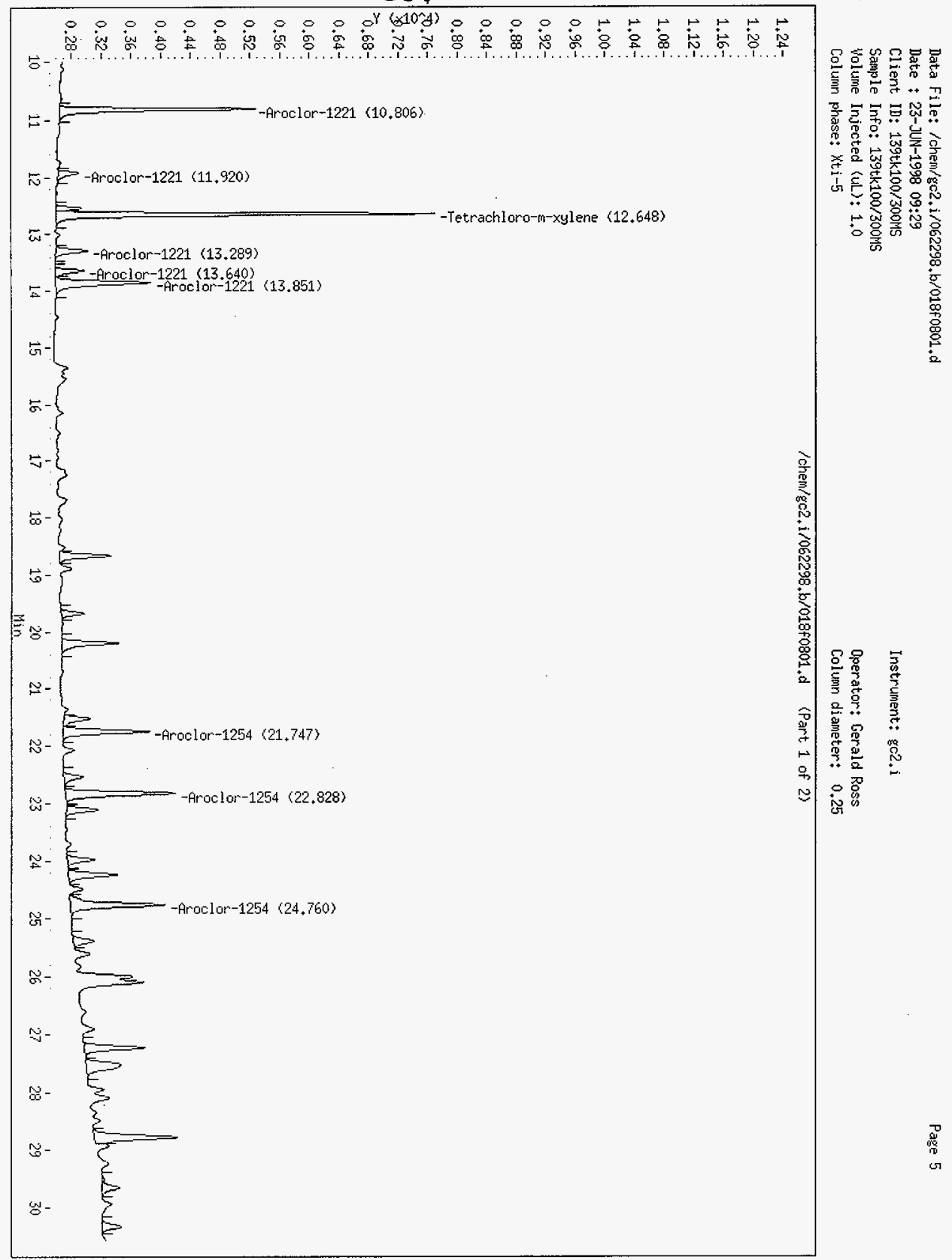


Date : 23-JUN-1998 09:29

Client ID: 139 tk100/300Ms

Sample Info: $139 \mathrm{tk} 100 / 300 \mathrm{HS}$

Volune Injected $\langle u L\rangle \neq 1,0$

Instrument: $\mathrm{gc} 2 . \mathrm{i}$

Colurnn phase: $x \mathrm{ti}-5$

Operator: Gerald Ross

Column diametert $\quad 0.25$

/chem/gc2,i/062298,b/018f0801,d (Part 2 of 2)

$1.2-$

$1.1-^{\circ}$

$1.0-$

$0.9-$

$0.8-$

$0.7-$

宫

守

$=-$

$0.4-$

0.4

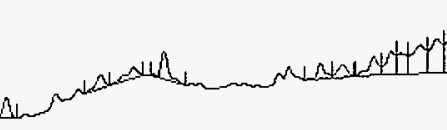

$0.3-$

$0.2-$

$0.1-$

$0.0-$

31

$32 \quad 33$

33

35

36

$37 \quad 38$

39

40

41

42

$44 \quad 45$

46

47

48

4

50 
Data File: /chem/gc2.i/062298.b/019f1001.d

Report Date: 20-Jul-1998 06:25

\section{2-S Laboratory}

Data file : /chem/gc2.i/062298.b/019f1001.d

Lab Smp Id: S98B0000220MSD

Inj Date : 23-JUN-1998 11:25

operator : Gerald Ross

Smp Info : 141tk100/300MSD

Misc Info: $141 \mathrm{tk100/300MSD}$

comment :

Method : /chem/gc2.i/062298.b/rcrapcb.m

Meth Date : 17-Jul-1998 09:20 gar

Cal Date : 22-JUN-98 19:59

Als bottle: 1

Dil Factor: 300.000

Integrator: HP Genie

Target Version: 3.10
Client Smp ID: 141tk100/300MSD

Inst ID: $9 \subset 2 . i$

Quant Type: ESTD

Cal File: $007 \mathrm{f} 0301 . \mathrm{d}$

QC Sample: MSD

Compound Sublist: Aroclors.sub Sample Matrix: SOIL

\begin{tabular}{|c|c|c|c|c|c|c|}
\hline \multirow[b]{3}{*}{ RT } & \multirow[b]{3}{*}{ EXP RT DLT RT } & & \multicolumn{2}{|c|}{ CONCENTRATIONS } & \multirow[b]{3}{*}{ TARGET RANGE } & \multirow{4}{*}{$\begin{array}{l}\text { RATIO } \\
====\end{array}$} \\
\hline & & & $\mathrm{ON}-\mathrm{COL}$ & FINAL & & \\
\hline & & RESPONSE & ( $n g)$ & (ug/Kg) & & \\
\hline$==$ & 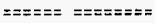 & $==ニ=ニ==0$ & $==== \pm=$ & $==== \pm==$ & 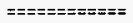 & \\
\hline & clor-1016 & & & CAS \# & $12674-11-2$ & \\
\hline
\end{tabular}

Peaks not detected for Quant. or Qual. signal(s).

23 Aroclor -1221

$\begin{array}{lll}10.811 & 10.811 & 0.000\end{array}$

$11.923 \quad 11.923 \quad 0.000$

$\begin{array}{lll}13.291 & 13.282 & 0.009\end{array}$

$\begin{array}{llll}13.643 & 13.673 & -0.030\end{array}$

$\begin{array}{lll}13.853 & 13.849 & 0.004\end{array}$

Average of Peak Concentrations

24 Aroclor -1232

Peaks not detected for Quant. or Qual, signal(s).

25 Aroclor $\cdot 1242$

$\begin{array}{rr}0 & \\ 0 & \\ 8306 & 0.0422 \\ 7492 & 0.0332 \\ 28320 & 0.0568 \\ \text { Concentrations }=\end{array}$

CAS \#: 11104-28-2

$14100208.00-258.00 \quad 0.00$

$11100 \quad 0.00-\quad 0.00 \quad 0.00$

$18900 \quad 0.00-\quad 0.00 \quad 0.00$

CAS \#: $1114-16-5$

Peaks not detected for Quant. or Qual, signal(s).

26 Aroclor -1248

CAS \#: $12672-29-6$

Operator disabled compound ident ification.

\begin{tabular}{|c|c|c|c|c|c|c|c|c|c|}
\hline \multicolumn{6}{|c|}{27 Aroclor -1254} & \multicolumn{4}{|c|}{ CAS \#: $11097-69-1$} \\
\hline 21.747 & 21.767 & -0.020 & $\cdot$ & 35792 & 0.0439 & 14600 & & & $100.00(R)$ \\
\hline 22.828 & 22.846 & -0.018 & & 50847 & 0.0471 & 15700 & $112.00-$ & 152.00 & 142.06 \\
\hline 24.760 & 24.780 & -0.020 & & 45687 & 0.0472 & 15700 & $96.00=$ & 136.00 & 127.65 \\
\hline \multicolumn{6}{|c|}{ Average of Pea } & \multicolumn{4}{|l|}{15400} \\
\hline
\end{tabular}


Data File: /chem/gc2.i/062298.b/019f1001.d

Report Date: 20-Jul-1998 06:25

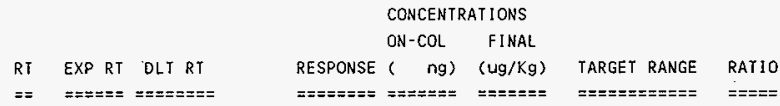

28 Aroclor -1260

CAS \#: $11096-82-5$

Operator disabled compound identification.

$\$ 29$ Tetrachloro-m-xylene

CAS \#: 877-09-8

$\begin{array}{lll}12.649 & 12.654 & -0.005\end{array}$

$921040.00625 \quad 2080$

100.00

$\$ 30$ Decachlorobiphenyl

CAS \#: $2051-24-3$

$\begin{array}{lll}42.686 & 42.728 & -0.042\end{array}$

$855340.0105 \quad 3500$

$100.00(R)$

QC Flag Legend

R - Spike/Surrogate failed recovery limits.

M - Compound response manually integrated. 
Data File: /chem/gc2.i/062298.b/019f1001.d

Report Date: 20-Jul-1998 06:25

\section{2-S Laboratory}

RECOVERY REPORT

Client Name:

Sample Matrix: SOLID

Lab Smp Id: S98B0000220MSD

Level: LoW

Data Type: GC MULTI COMP

Spikelist File: Aroclor-1254.spk

Method

Misc Info: 141 tk100/300MSD
Client SDG: 062298

Fraction: PEST

Client Smp ID: 141tk100/300MSD

Operator: Gerald Ross

SampleType: MSD

Quant Type: ESTD

\begin{tabular}{|c|c|c|c|c|}
\hline SPIKE COMPOUND & $\begin{array}{l}\text { CONC } \\
\text { ADDED } \\
u g / K g\end{array}$ & $\begin{array}{c}\text { CONC } \\
\text { RECOVERED } \\
\text { ug } / \mathrm{Kg}\end{array}$ & RECOVERED & LIMITS \\
\hline 27 Aroclor -1254 & 8890 & $\begin{aligned} & 15400 \\
- & 8430\end{aligned}$ & $-172.85 \pi$ & $\overline{50-150}$ \\
\hline & & $1000 \times 6970 / 88$ & $=78.40 \%$ & $-h$ \\
\hline SURROGATE COMPOUND & $\begin{array}{l}\text { CONC } \\
\text { ADDED } \\
\mathrm{ug} / \mathrm{Kg}\end{array}$ & $\begin{array}{c}\text { CONC } \\
\text { RECOVERED } \\
\mathrm{ug} / \mathrm{Kg}\end{array}$ & $\begin{array}{c}\% \\
\text { RECOVERED }\end{array}$ & LIMITS \\
\hline $\begin{array}{lll}\$ & 29 & \text { Tetrachloro-m-xyle } \\
\$ & 30 & \text { Decachlorobipheny }\end{array}$ & $\begin{array}{l}2220 \\
2220\end{array}$ & $\begin{array}{l}2080 \\
3500\end{array}$ & $\begin{array}{c}93.69 \\
157.71 *\end{array}$ & $\begin{array}{l}50-150 \\
50-150\end{array}$ \\
\hline
\end{tabular}


Data File: /chem/gc2.i/062298.b/019f1001.d

Report Date: 20-Jul-1998 06:25

$$
\begin{aligned}
& \text { 222-S Iaboratory } \\
& \text { TARGET COMPOUNDS }
\end{aligned}
$$

Client Name:

Lab Smp Id: S98B0000220MSD

Sample Location:

Sample Date:

Sample Matrix: SOIL

Analysis Type: PEST

Data Type: GC MULTI COMP

Misc Info: 141tk100/300MSD
Client SDG: 062298

Client Smp ID: 141tk100/300MSD

Sample Point:

Date Received:

Quànt Type: ESTD

Level: LOW

Operator: Gerald Ross

CONCENTRATION UNITS:

(ug/L or ug/KG) ug/Kg

\begin{tabular}{|c|c|c|}
\hline $12674-11-2----$ Aroclor -1016 & 9900 & $\mathrm{U}$ \\
\hline $11104-28-2-----$ Aroclor -1221 & 14700 & \\
\hline $1114-16-5-----$ Aroclor -1232 & 9900 & $\mathrm{U}$ \\
\hline $53469-21-9----$ Aroclor -1242 & 9900 & $\mathrm{U}$ \\
\hline $12672-29-6 \cdots-$ Aroclor -1248 & 9900 & $\mathrm{U}$ \\
\hline $11097-69-1-\cdots-$ Aroclor $-1254^{-}$ & 15400 & \\
\hline $11096-82-5-----$ Aroclor -1260 & 9900 & $\mathbf{U}$ \\
\hline 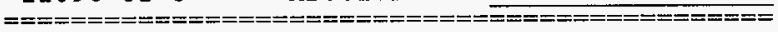 & $======\mathrm{=}=\mathrm{m}=\mathrm{m}=$ & $=====$ \\
\hline 877-09-8------Tetrachloro-m-xylene & 2080 & \\
\hline $2051-24-3---\ldots-$ Decachlorobiphenyl & 3500 & \\
\hline
\end{tabular}




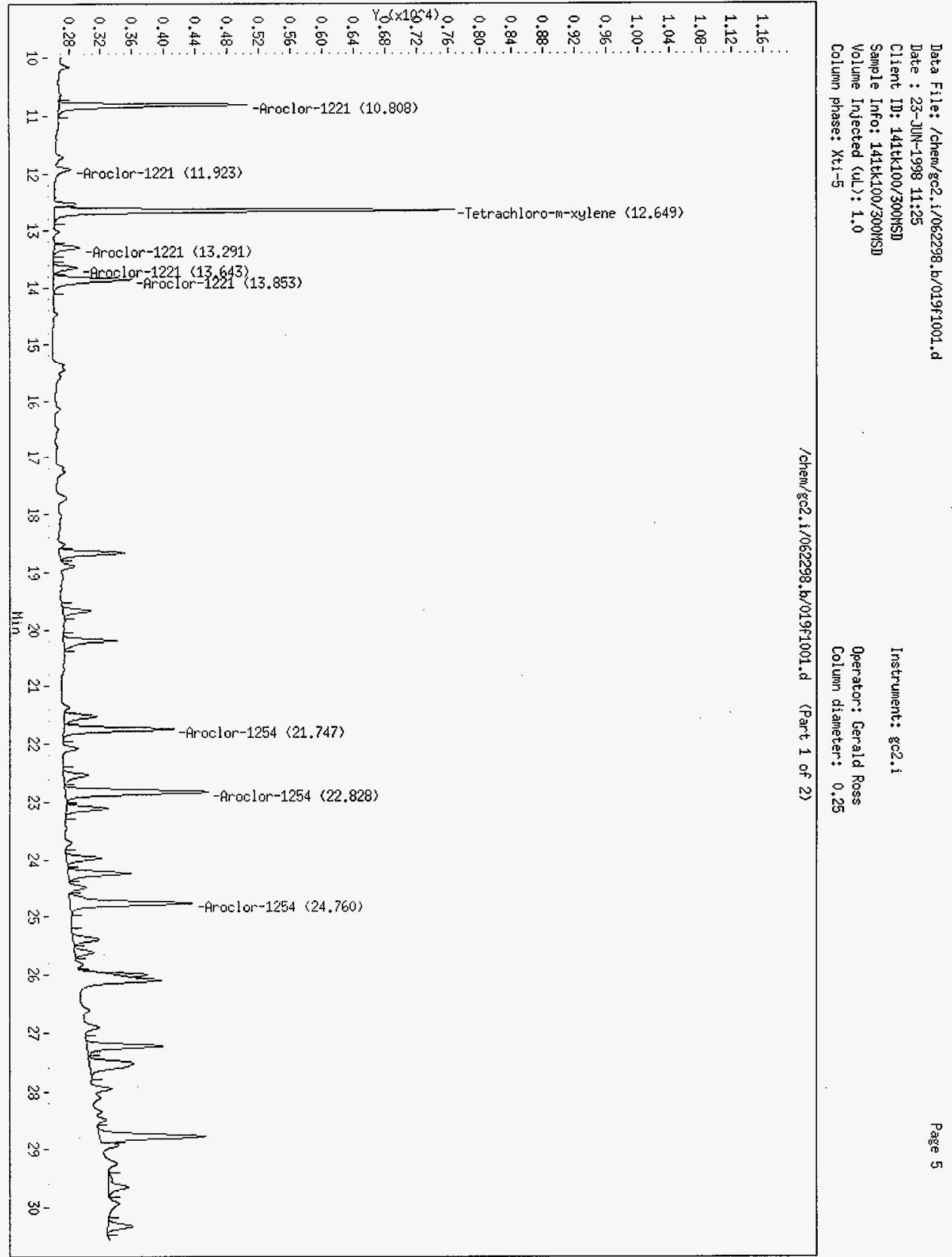


HNF-SD-WM-DP-309 REV. 0

0
4
5
0

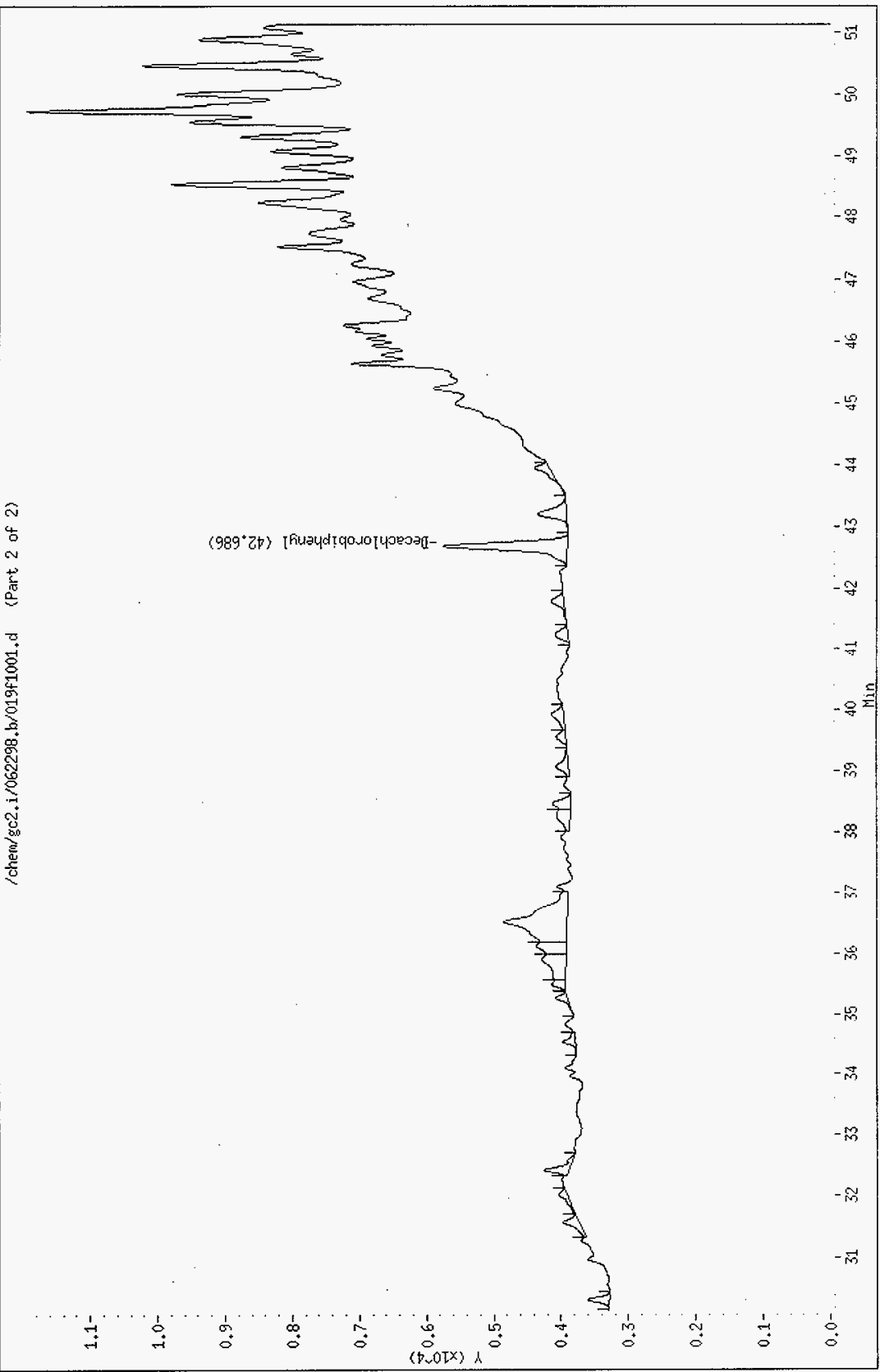

109 


\section{2-S Laboratory}

Data file: /chem/gc2.i/062298.b/020f1201.d

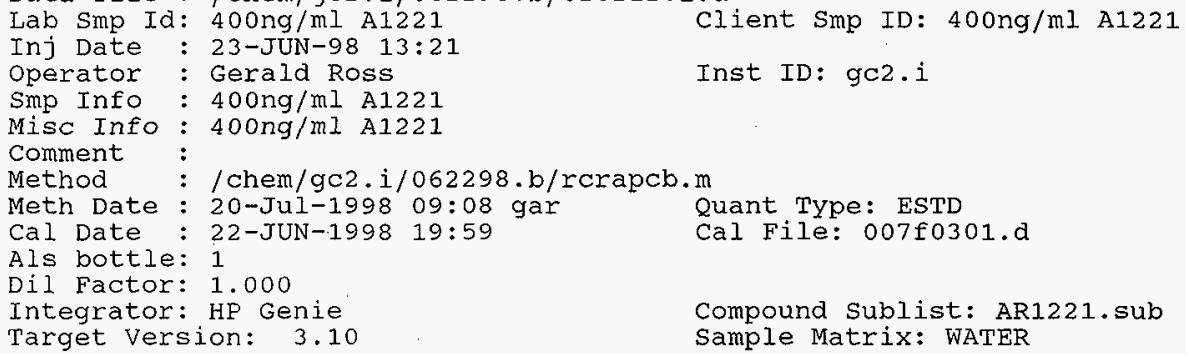

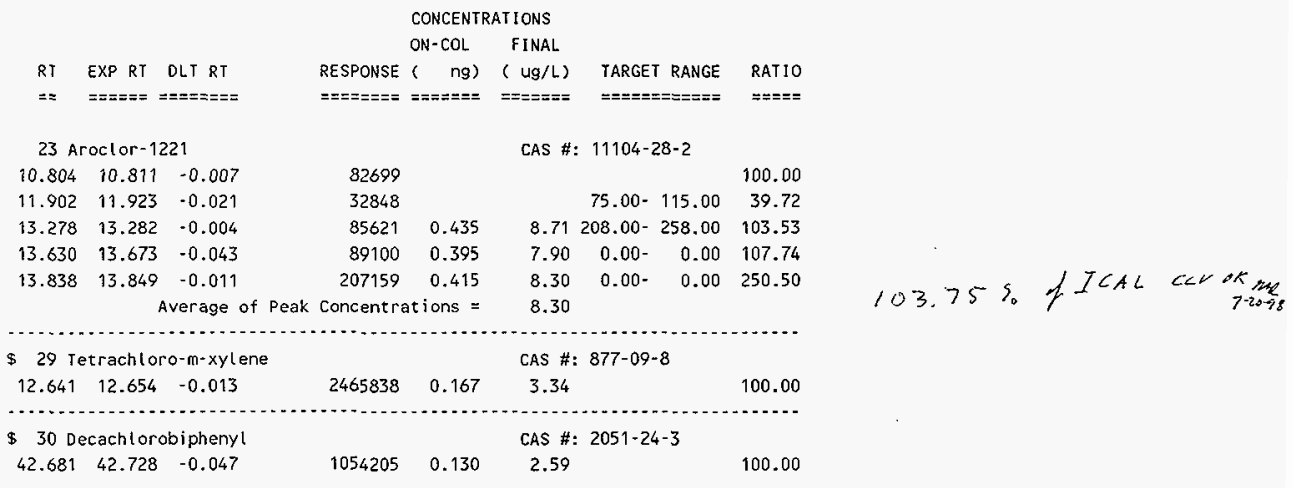


Data File: /chem/gc2.i/062298.b/020f1201.d

Report Date: 20-Jul-1998 09:29

$$
\text { 222-S Laboratory }
$$

RECOVERY REPORT

Client Name:

Sample Matrix: LIQUID

Lab Smp Id: 400ng/ml A1221

Level: LOW

Data Type: GC MULTI COMP

Spikelist File: Aroclor $-1660 . \mathrm{spk}$

Method File:/chem/gc2.i/062298.b/rcrapcb.m

Misc Info: $400 \mathrm{ng} / \mathrm{mI}$ A1221

\begin{tabular}{|c|c|c|c|c|}
\hline SURROGATE COMPOUND & $\begin{array}{l}\text { CONC } \\
\text { ADDED } \\
\text { ug/L }\end{array}$ & $\begin{array}{c}\text { CONC } \\
\text { RECOVERED } \\
\text { ug/L }\end{array}$ & $\begin{array}{c}\% \\
\text { RECOVERED }\end{array}$ & LIMITS \\
\hline $\begin{array}{lll} & 29 & \text { Tetrachloro-m-xyle } \\
\$ & 30 \text { Decachlorobiphenyl }\end{array}$ & $\begin{array}{l}4.00 \\
4.00\end{array}$ & $\begin{array}{l}3.34 \\
2.59\end{array}$ & $\begin{array}{l}83.61 \\
64.79\end{array}$ & $\begin{array}{l}50-150 \\
50-150\end{array}$ \\
\hline
\end{tabular}

Client SDG: 062298

Fraction: PEST

Client Smp ID: $400 \mathrm{ng} / \mathrm{ml}$ A1221

operator: Gerald Ross

SampleType: SAMPLE

Quant Type: ESTD 
Data File: /chem/gc2.i/062298.b/020f1201.d

Report Date: 20-Jul-1998 09:29

$$
\begin{aligned}
& \text { 222-S Laboratory } \\
& \text { TARGET COMPOUNDS }
\end{aligned}
$$

Client Name:

Lab Smp Id: 400ng/ml A1221

Sample Location:

Sample Date:

Sample Matrix: WATER

Analysis Type: PEST

Data Type: GC MULTI COMP

Misc Info: $400 \mathrm{ng} / \mathrm{ml}$ A1221
Client SDG: 062298

Client Smp ID: $400 \mathrm{ng} / \mathrm{ml}$ A1221 Sample Point:

Date Received:

Quant Type: ESTD

Level: LOW

Operator: Gerald Ross

\section{CONCENTRATION UNITS:}

(ug/L or $\mathrm{ug} / \mathrm{KG}$ ) ug/L

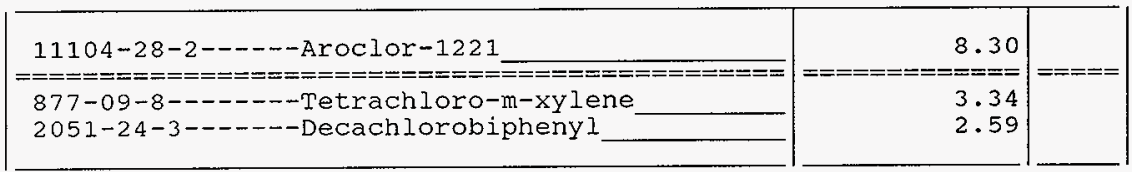




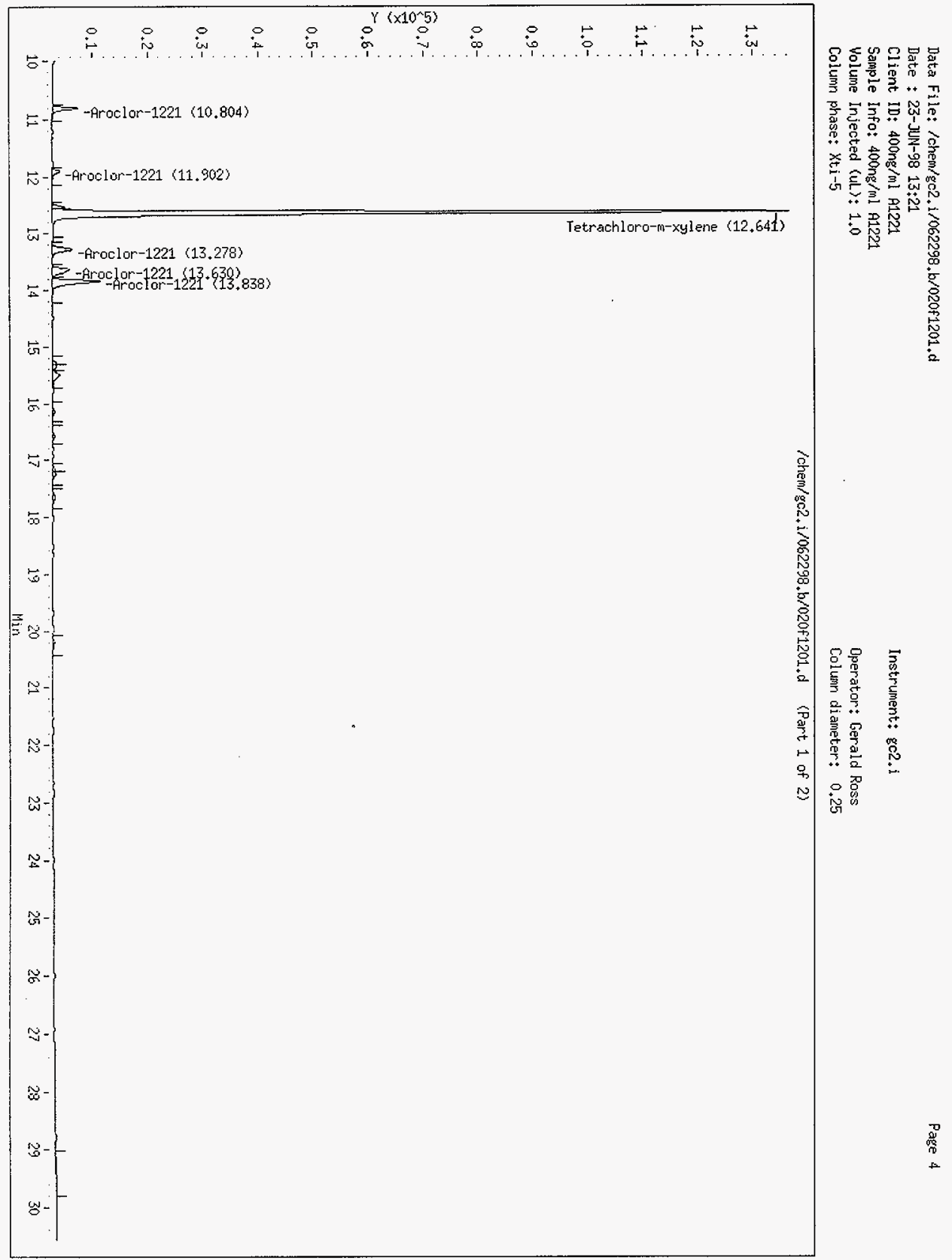


Data File: /chem/gc2,i/062298,b/020f1201.d

Gate : 23-JuN-98 13:21

Client 1D: 400ng/ml A1221

Sample Info: 400ng/ml A1221

Volume Injected (uL): 1,0

Instrument: $g c 2, \mathrm{i}$

Column phase: $X t i-5$

Operator: Gerald Ross

Columin diameter: 0.25

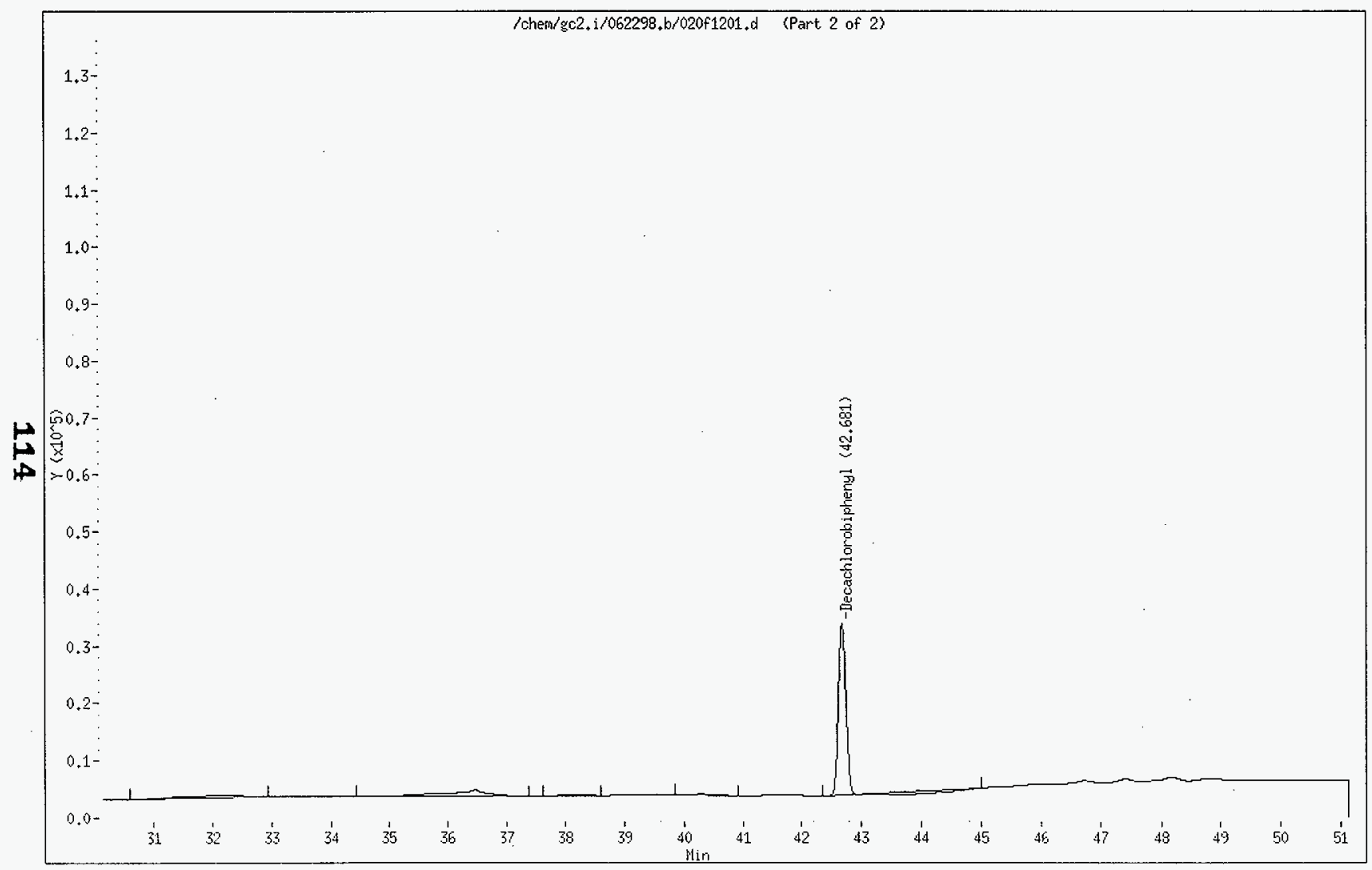


Data File: /chem/gc2.i/062298.b/021f1201.d

Page 1

Report Date: 29-Jun-1998 15:02

\section{2-S Laboratory}

Data file : /chem/gc2.i/062298.b/021f1201.d

Lab Smp Id: $200 \mathrm{ng} / \mathrm{ml}$ A 1660

Client Smp ID: 200ng/ml A1660

Inj Date : 23-JUN-1998 14:19

Operator : Gerald Ross

Smp Info : $200 \mathrm{ng} / \mathrm{ml}$ A1660

Inst ID: $g \mathrm{C} 2 . \mathrm{i}$

Misc Info : $200 \mathrm{ng} / \mathrm{ml}$ A 1660

Comment :

Method : /chem/gc2.i/062298.b/rcrapcb.m

Meth Date : 24-Jun-1998 09:58 gar

Cal Date : 22-JUN-98 19:59

Als bottle: 1

Dil Factor: 1.000

Integrator: HP Genie

Target Version: 3.10

Quant Type: ESTD

Cal File: $007 f 0301 . d$

QC Sample: METHSPIKE

Compound sublist: AR1660.sub Sample Matrix: WATER

\begin{tabular}{|c|c|c|c|c|c|c|c|}
\hline & & & & CONCENTR & ATIONS & & \\
\hline & & & & $\mathrm{ON}-\mathrm{COL}$ & FINAL & & \\
\hline RT & EXP RT & DLT RT & RESPONSE & ( $n g)$ & $(\mathrm{ug} / \mathrm{L})$ & TARGET RANGE & RATIO \\
\hline$==$ & $====\pi==$ & $\Rightarrow= \pm==\pi==$ & $======\Xi$ & $== \pm====$ & $==\pi==\pi=$ & 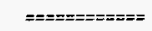 & $=\approx==0$ \\
\hline $22 \mathrm{Ar}$ & roclor-10 & 016 & & & CAS \#: & $12674-11-2$ & \\
\hline 15.353 & 15.374 & -0.021 & 128437 & 0.198 & 3.97 & & 100.00 \\
\hline 17.148 & 17.171 & -0.023 & 138164 & 0.187 & 3.75 & $114.00-134.00$ & 107.57 \\
\hline 17.225 & 17.247 & -0.022 & 191522 & 0.203 & 4.06 & $134.00-154.00$ & 149.12 \\
\hline & & Average of Peak & Concentra & ations n & 3.93 & & \\
\hline $28 \mathrm{Ar}$ & roclor-12 & & & & CAS \#: & $11096-82-5$ & \\
\hline 25.989 & 26.019 & -0.030 & 188487 & 0.194 & 3.87 & & 100.00 \\
\hline 27.223 & 27.253 & -0.030 & 183541 & 0.190 & 3.79 & $89.00-109.00$ & 97.38 \\
\hline 28.770 & 28.800 & -0.030 & 172167 & 0.194 & 3.88 & $72.00-92.00$ & 91.34 \\
\hline & & Average of Peak & Concentre & ations = & 3.85 & & \\
\hline$\$ 29 \mathrm{re}$ & etrachlor & ro-m-xylene & & & CAS \#: & $877-09-8$ & \\
\hline 12.640 & 12.654 & -0.014 & 1628593 & 0.110 & 2.21 & & 100.00 \\
\hline$\$ 30 \mathrm{De}$ & ecachloro & obiphenyl & & & CAS \#: & $2051-24-3$ & \\
\hline 42.678 & 42.728 & -0.050 & 745969 & 0.0917 & 1.83 & & 100.00 \\
\hline
\end{tabular}


Data File: /chem/gc2.i/062298.b/021f1201.d

Report Date: 29-Jun-1998 15:02

\section{2-S Laboratory}

RECOVERY REPORT

Client Name:

Sample Matrix: LIQUID

Lab Smp Id: $200 \mathrm{ng} / \mathrm{ml}$ A1660

Level: LOW

Data Type: GC MULTI COMP

Spikelist File: Aroclor-1660.spk

Method file: /chem/gc2.i/052298.

Misc Info: $200 \mathrm{ng} / \mathrm{ml} \mathrm{A} 1660$
Client SDG: 062298

Fraction: PEST

Client Smp ID: $200 \mathrm{ng} / \mathrm{ml}$ A1660

Operator: Gerald Ross

SampleType: METHSPIKE

Quant Type: ESTD

\begin{tabular}{|c|c|c|c|c|}
\hline SPIKE COMPOUND & $\begin{array}{l}\text { CONC } \\
\text { ADDED } \\
\text { ug/I }\end{array}$ & $\begin{array}{l}\text { CONC } \\
\text { RECOVERED } \\
\text { ug/L }\end{array}$ & RECOVERED & LIMITS \\
\hline $\begin{array}{l}22 \text { Aroclor }-1016 \\
28 \text { Aroclor }-1260\end{array}$ & $\begin{array}{l}4.00 \\
4.00\end{array}$ & $\begin{array}{l}3.93 \\
3.85\end{array}$ & $\begin{array}{l}98.16 \\
96.20\end{array}$ & $\begin{array}{l}50-150 \\
50-150\end{array}$ \\
\hline SURROGATE COMPOUND & $\begin{array}{l}\text { CONC } \\
\text { ADDED } \\
\text { ug/I }\end{array}$ & $\begin{array}{c}\text { CONC } \\
\text { RECOVERED } \\
u g / L\end{array}$ & $\begin{array}{c}\stackrel{\%}{\circ} \\
\text { RECOVERED }\end{array}$ & LIMITS \\
\hline $\begin{array}{lll}\$ & 29 & \text { Tetrachloro-m-xyle } \\
\$ & 30 \text { Decachlorobiphenyl }\end{array}$ & $\begin{array}{l}2.00 \\
2.00\end{array}$ & $\begin{array}{l}2.21 \\
1.83\end{array}$ & $\begin{array}{r}110.45 \\
91.69\end{array}$ & $\begin{array}{l}\overline{50-150} \\
50-150\end{array}$ \\
\hline
\end{tabular}


Data File: /chem/gc2.i/062298.b/021f1201.d

Report Date: 29-Jun-1998 15:02

222-S Laboratory

TARGET COMPOUNDS

Client Name:

Lab Smp Id: $200 \mathrm{ng} / \mathrm{ml}$ A 1660

Sample Location:

Sample Date:

Sample Matrix: WATER

Analysis Type: PEST

Data Type: GC MULTI COMP

Misc Info: $200 \mathrm{ng} / \mathrm{ml}$ A 1660
Client SDG: 062298

Client Smp ID: $200 \mathrm{ng} / \mathrm{ml}$ A1660

Sample Point:

Date Received:

Quant Type: ESTD

Level: LOW

Operator: Gerald Ross
CAS NO.

COMPOUND
CONCENTRATION UNITS:

( $u g / L$ or $u g / K G) ~ u g / L$

\begin{tabular}{|r|r|}
$12674-11-2------$ Aroclor -1016 & 3.93 \\
$11096-82-5------$ Aroclor -1260 & 3.85 \\
$====================================$ \\
$877-09-8-------$ Tetrachloro-m-xylene
\end{tabular} \mid$==============$




\section{$8 \tau \tau$}

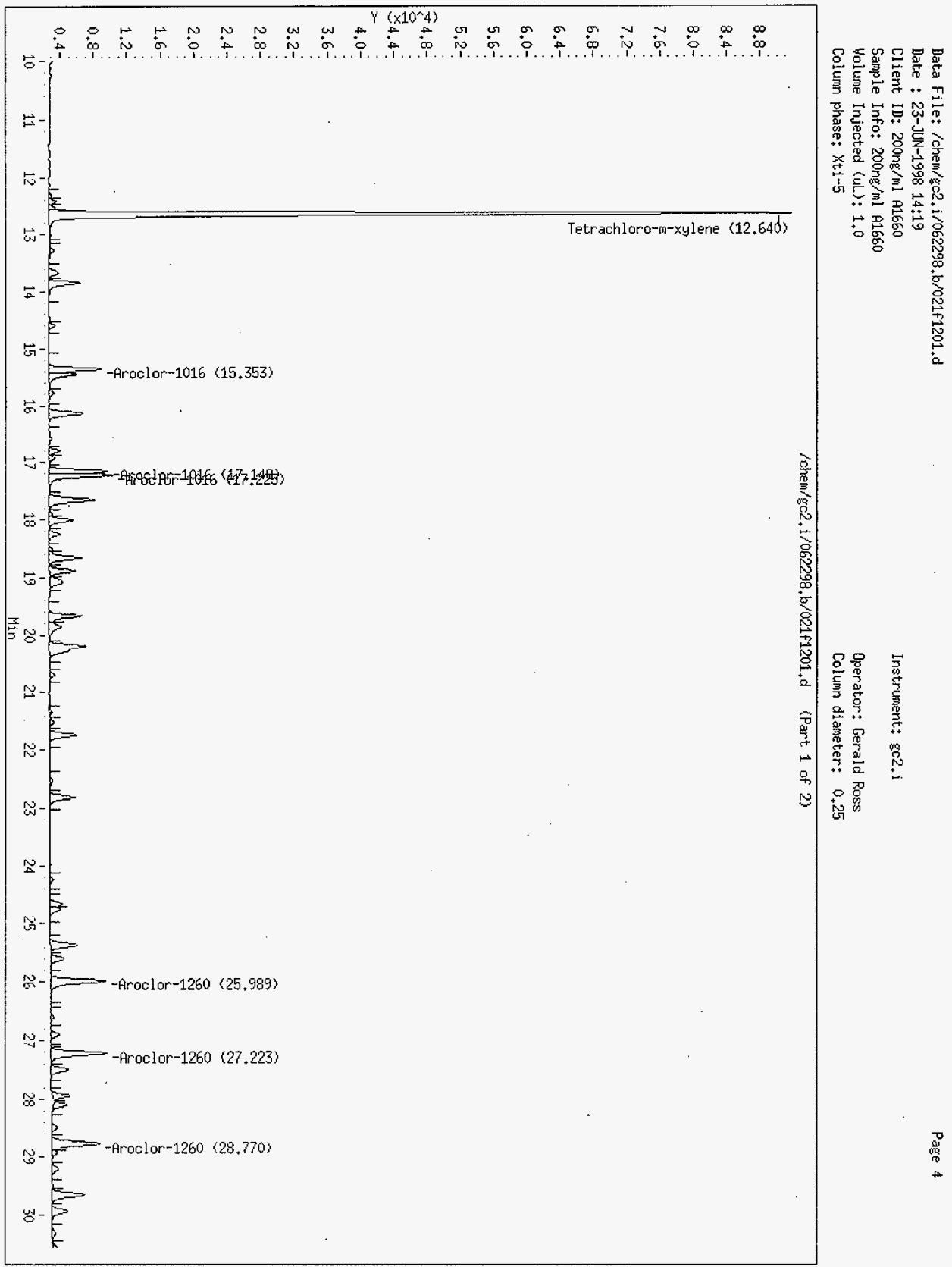


- 23-Juk-1998 14*19

Client ID: 200ng/ml A1660

Sample Info: 200ng/ml A1660

Volume Injected (uL): 1,0

Column phase: Xti-5

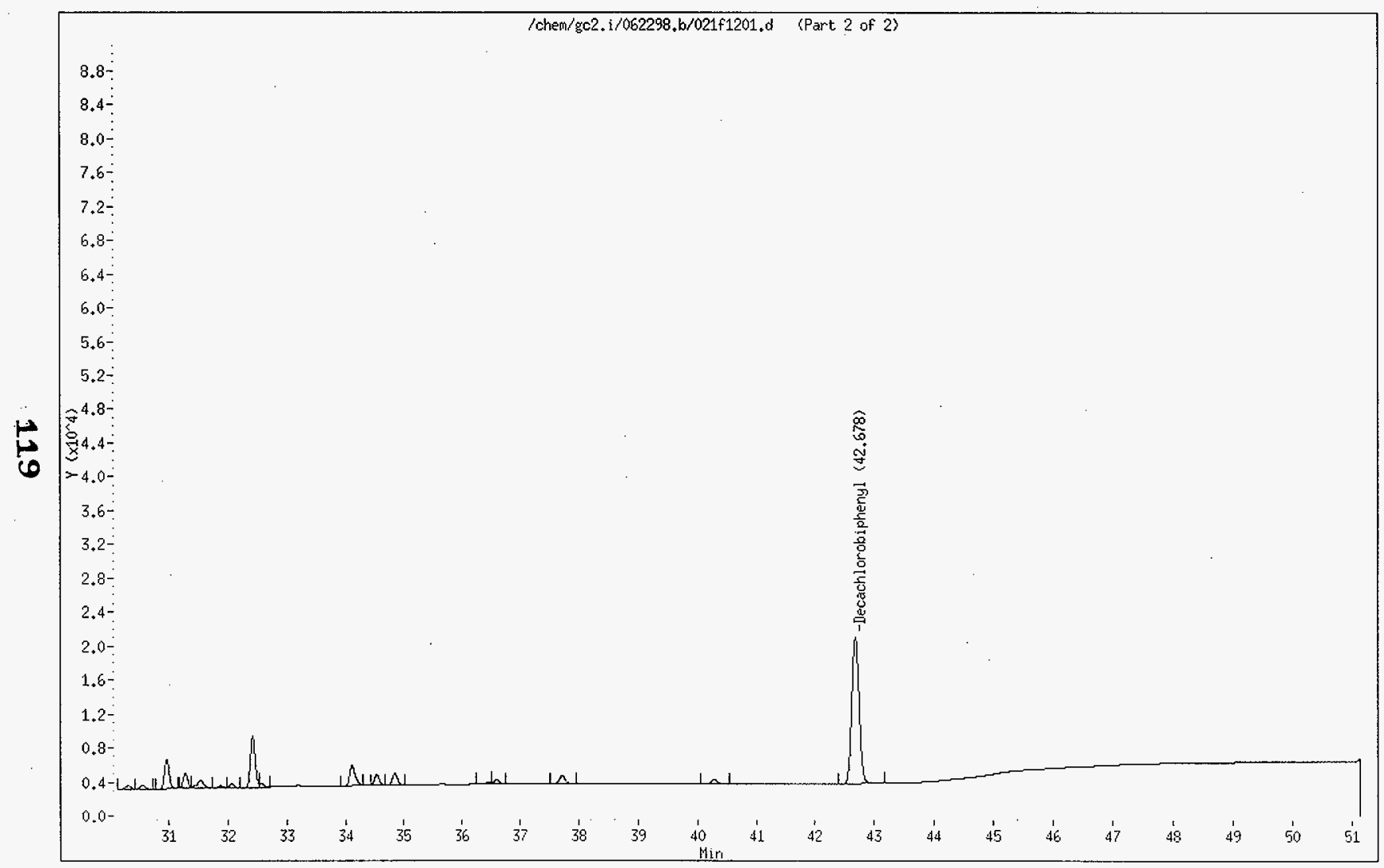

Instrument: gc2, i

Operator: Gerald Ross

Operator: Gerald Ross 
Data File: /chem/gc2.i/062298.b/022f1201.d

Report Date: 29-Jun-1998 13:41

\section{2-S Laboratory}

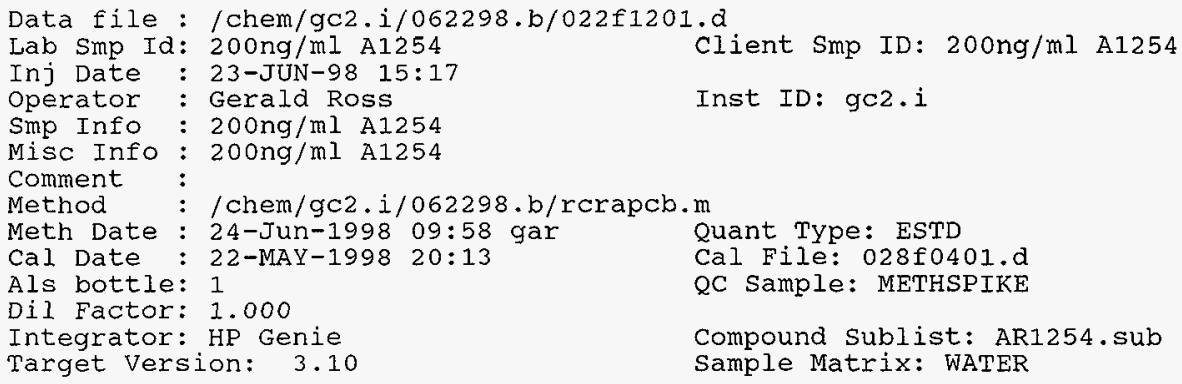

\begin{tabular}{|c|c|c|c|c|c|c|c|}
\hline \multicolumn{8}{|c|}{ CONCENTRATIONS } \\
\hline \multirow[b]{2}{*}{ RT } & \multirow{2}{*}{\multicolumn{2}{|c|}{ EXP RT DLI RT }} & \multirow[b]{2}{*}{ RESPONSE } & $\mathrm{ON}-\mathrm{COL}$ & FINAL & \multirow[b]{2}{*}{ TARGET RANGE } & \multirow[b]{2}{*}{ RATIO } \\
\hline & & & & ( $\mathrm{ng})$ & $(u g / L)$ & & \\
\hline$==$ & $=\boldsymbol{s}=\boldsymbol{=}=\boldsymbol{=}$ & 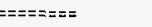 & $===ニ===+$ & $==ニ===-$ & $====+=\Omega$ & $===ニ==ニ=ニ=ニ=$ & $== \pm==$ \\
\hline \multicolumn{3}{|c|}{27 Aroclor-1254 } & & \multicolumn{4}{|c|}{ CAS \#: $11097-69-1$} \\
\hline 21.741 & 21.767 & -0.026 & 173483 & 0.213 & 4.26 & & 100.00 \\
\hline 22.820 & 22.846 & -0.026 & 225475 & 0.209 & 4.18 & $112.00-152.00$ & 129.97 \\
\hline 24.753 & 24.780 & -0.027 & 213414 & 0.220 & 4.41 & $96.00-136.00$ & 123.02 \\
\hline \multicolumn{3}{|c|}{ Average of Peak } & \multicolumn{3}{|c|}{ Concentrations $=$} & & \\
\hline \multicolumn{4}{|c|}{$\$ 29$ Tetrachloro-m-xylene } & \multicolumn{4}{|c|}{ CAS \#: $877=09-8$} \\
\hline 12.642 & 12.654 & -0.012 & 1391748 & 0.0944 & 1.89 & & 100.00 \\
\hline \multicolumn{4}{|c|}{$\$ 30$ Decachlorobiphenyl } & \multicolumn{4}{|c|}{ CAS \#: $2051-24-3$} \\
\hline 42.679 & 42.728 & -0.049 & 904473 & 0.111 & 2.22 & & 100.00 \\
\hline
\end{tabular}


Data File: /chem/gc2.i/062298.b/022f1201.d

Report Date: 29-Jun-1998 13:41

$$
\begin{aligned}
& \text { 222-S Laboratory } \\
& \text { RECOVERY REPORT }
\end{aligned}
$$

Client Name:

Sample Matrix: LIQUID

Lab Smp Id: $200 \mathrm{ng} / \mathrm{ml}$ A1254

Level: LoW

Data Type: GC MUITI COMP

Spikelist File: Aroclor-1254.spk

Method File: /chem/gc2 i/062298.b/

Misc Info: $200 \mathrm{ng} / \mathrm{ml}$ A1254
Client SDG: 062298

Fraction: PEST

Client Smp ID: 200ng/ml A1254 Operator: Gerald Ross

SampleType: METHSPIKE

\begin{tabular}{|c|c|c|c|c|}
\hline SPIKE COMPOUND & $\begin{array}{l}\text { CONC } \\
\text { ADDED } \\
u g / L\end{array}$ & $\begin{array}{l}\text { CONC } \\
\text { RECOVERED } \\
\mathrm{ug} / \mathrm{L}\end{array}$ & $\stackrel{\%}{\%}$ RECOVERED & LIMITS \\
\hline 27 Aroclor -1254 & 4.00 & 4.28 & 107.08 & $\overline{50-150}$ \\
\hline SURROGATE COMPOUND & $\begin{array}{l}\text { CONC } \\
\text { ADDED } \\
\mathrm{ug} / \mathrm{L}\end{array}$ & $\begin{array}{l}\text { CONC } \\
\text { RECOVERED } \\
\text { ug/L }\end{array}$ & $\begin{array}{c}\% \\
\text { RECOVERED }\end{array}$ & LIMITS \\
\hline $\begin{array}{l}\$ 29 \text { Tetrachloro-m-xyle } \\
\$ 30 \text { Decachlorobiphenyl }\end{array}$ & $\begin{array}{l}2.00 \\
2.00\end{array}$ & $\begin{array}{l}1.89 \\
2.22\end{array}$ & $\begin{array}{r}94.39 \\
111.18\end{array}$ & $\begin{array}{l}50-150 \\
50-150\end{array}$ \\
\hline
\end{tabular}

Quant Type: ESTD 
Data File:/chem/gc2.i/062298.b/022f1201.d

Report Date: 29-Jun-1998 13:41

\section{2-S Laboratory \\ TARGET COMPOUNDS}

Client Name:

Lab Smp Id: $200 \mathrm{ng} / \mathrm{ml}$ A.1254

Sample Location:

Sample Date:

Sample Matrix: WATER

Analysis Type: PEST

Data Type: GC MULTI COMP

Misc Info: $200 \mathrm{ng} / \mathrm{ml}$ A1254 client SDG: 062298

Client Smp ID: $200 \mathrm{ng} / \mathrm{ml}$ A1254

Sample point:

Date Received:

Quant Type: ESTD

Level: LOW

Operator: Gerald Ross

CONCENTRATION UNITS:

(ug/L or $\mathrm{ug} / \mathrm{KG}$ ) ug/I

\begin{tabular}{|c|c|c|}
\hline $11097-69-1------$ Aroclor -1254 & 4.28 & \\
\hline 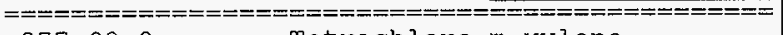 & $==========$ & $=====$ \\
\hline $\begin{array}{l}877-09-8------- \text { Tetrachloro-m-xylene } \\
2051-24-3------ \text { Decachlorobiphenyl }\end{array}$ & $\begin{array}{r}1.89 \\
2.22\end{array}$ & \\
\hline
\end{tabular}


Date : 23-JuN-98 15:17

Client ID: $200 \mathrm{ng} / \mathrm{ml}$ A1254

Sámple Info: 200ng/ml A1254

Volume Injected $(u L): 1,0$

Operator: Gerald Ross

Column phase: $x t i-5$

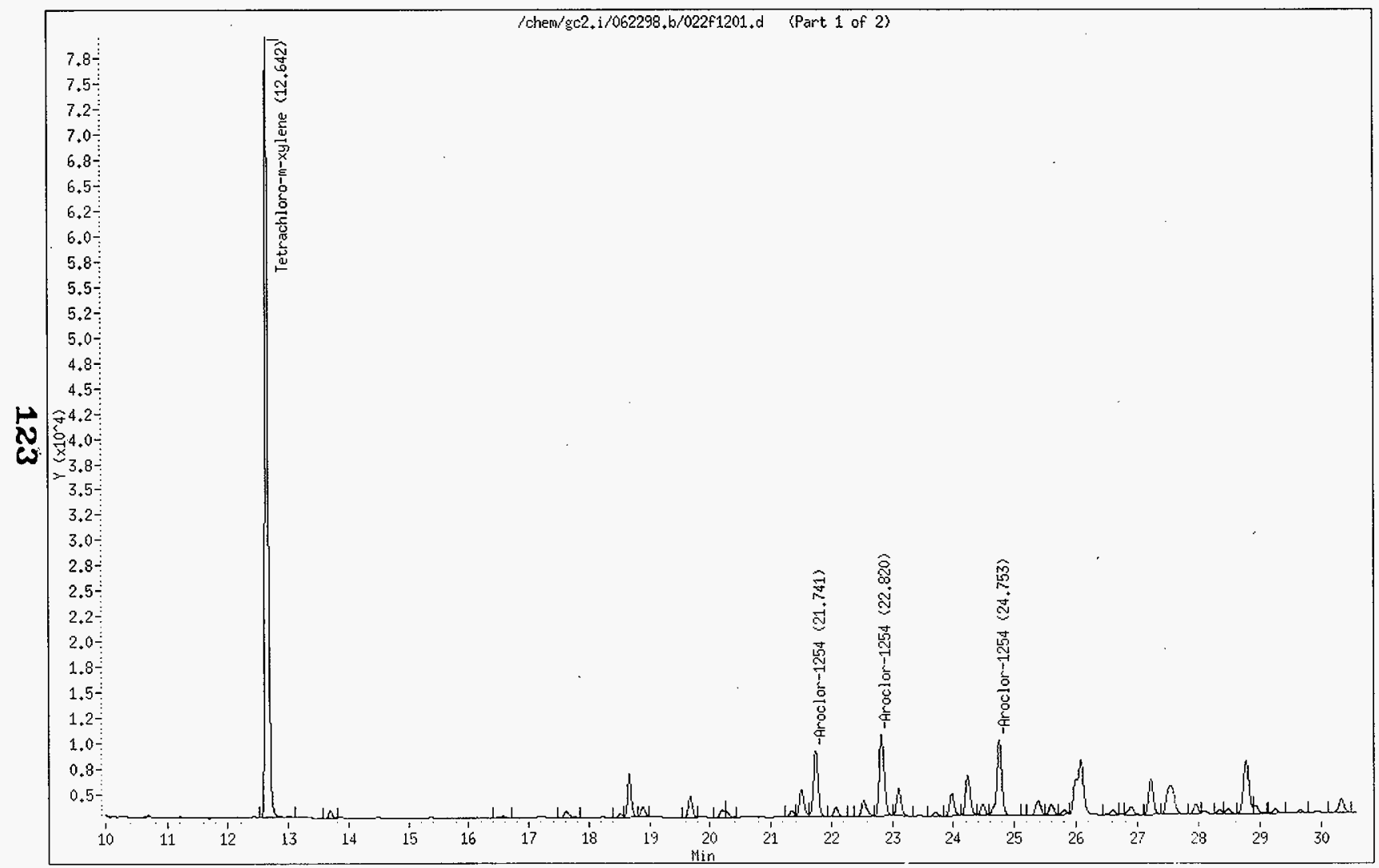




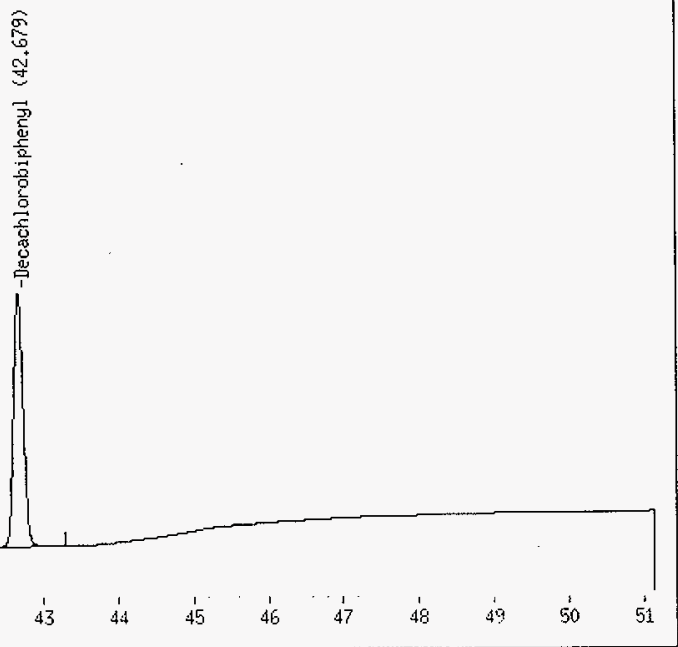




\section{HNF-SD-WM-DP-309 REV. 0}

Data File: /chem/gc2.i/062298.b/022f1301.d

Report Date: 29-Jun-1998 13:41

\section{2-S Laboratory}

Data file : /chem/gc2.i/062298.b/022f1301.d

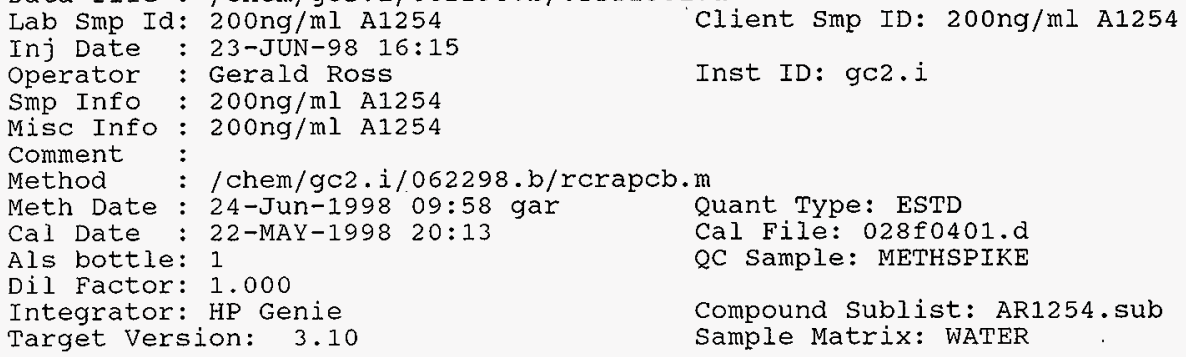

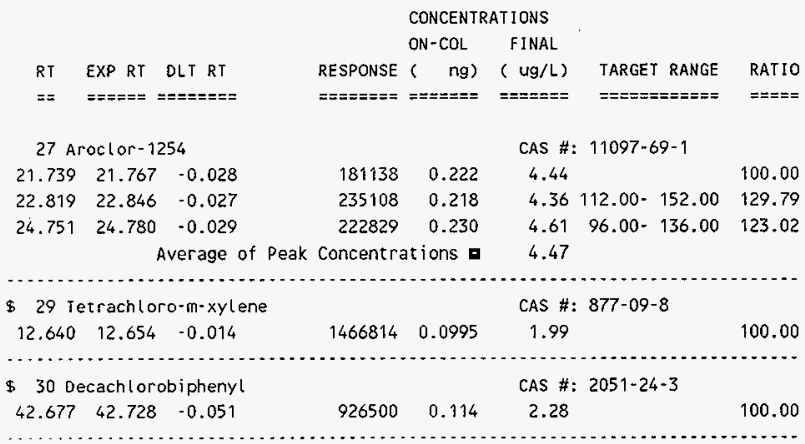




\section{HNF-SD-WM-DP-309 REV. 0}

Data File: /chem/gc2.i/062298.b/022f1301.d

Report Date: 29-Jun-1998 13:41

\section{2-S Laboratory}

RECOVERY REPORT

Client Name:

Sample Matrix: LIQUID

Lab Smp Id: $200 \mathrm{ng} / \mathrm{ml}$ A1254

Level: LOW

Data Type: GC MULTI COMP

Spikelist File: Aroclor-1254.spk

Method File: / chem/gc2.i/062298.b/rcrapcb.m

Misc Info: $200 \mathrm{ng} / \mathrm{ml}$ A1254

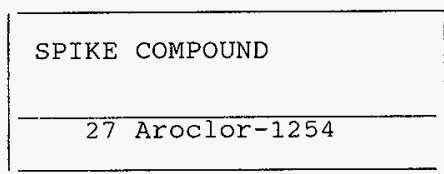

\begin{tabular}{l} 
CONC \\
ADDED $/ \mathrm{L}$ \\
\hline 4.00
\end{tabular}
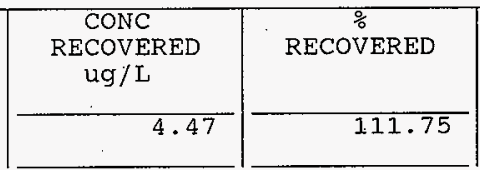

LIMITS

Fraction: PEST

Client Smp ID: $200 \mathrm{ng} / \mathrm{ml}$ A1254

Operator: Gerald Ross

SampleType: METHSPIKE

Quant Type: ESTD

\begin{tabular}{|c|c|c|c|c|}
\hline SURROGATE COMPOUND & $\begin{array}{l}\text { CONC } \\
\text { ADDED } \\
\mathrm{ug} / \mathrm{L}\end{array}$ & $\begin{array}{c}\text { CONC } \\
\text { RECOVERED } \\
\text { ug/L }\end{array}$ & RECOVERED & LIMITS \\
\hline $\begin{array}{lll}\text { \$ } 29 & \text { Tetrachloro-m-xyle } \\
\text { \$. } 30 \text { Decachlorobiphenyl }\end{array}$ & $\begin{array}{l}2.00 \\
2.00\end{array}$ & $\begin{array}{l}1.99 \\
2.28\end{array}$ & $\begin{array}{r}99.48 \\
113.88\end{array}$ & $\begin{array}{l}50-150 \\
50-150\end{array}$ \\
\hline
\end{tabular}


Data File: /chem/gc2.i/062298.b/022f1301.d

Report Date: 29-Jun-1998 13:41

222-S Laboratory

TARGET COMPOUNDS

Client Name:

Lab Smp Id: 200ng/ml A1254

Sample Location:

Sample Date:

Sample Matrix: WATER

Analysis Type: PEST

Data Type: GC MULTI COMP

$\mathrm{Misc}$ Info: $200 \mathrm{ng} / \mathrm{ml} \mathrm{A} 1254$
Client SDG: 062298

Client Smp ID: $200 \mathrm{ng} / \mathrm{ml}$ A1254

Sample Point:

Date Received:

Quant Type: ESTD

Level: LOW

Operator: Gerald Ross

CONCENTRATION UNITS:

(ug/L or $u g / K G$ ) ug/L

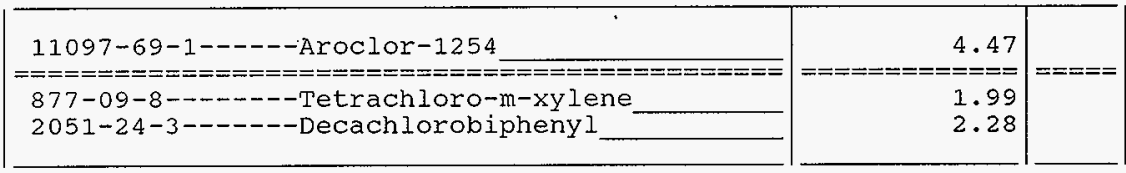


Date : 23-JuN -98 16:15

Client II: 200ng/ml A1254

Sample Info: 200ng/ml R1254

Uolume Injected (uL): 1.0

Instrument: $\mathrm{gc} 2+1$

Column phase: Kt $i-5$

Dperator: Gerald Ross

Column diameter: 0.25

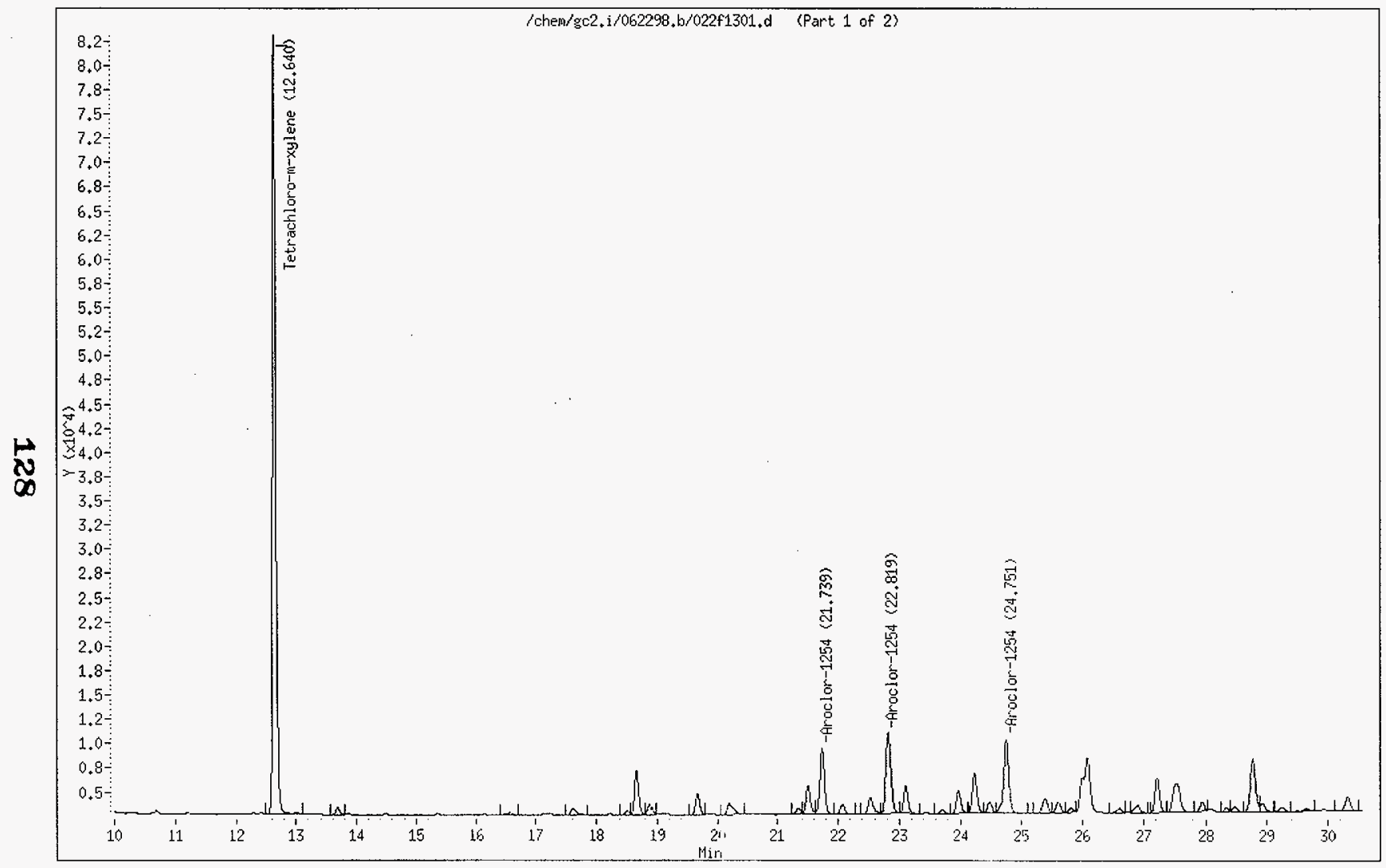


Date : 23-JuN -98 16:15

Client ID: 200ng/ml A1254

Sample Info: 200ng/ml A1254

Volume Injected (uL): 1.0

Operator: Gerald Ross

Column phase: $\mathrm{xt}, \mathrm{i}-5$

Column diameter: 0.25

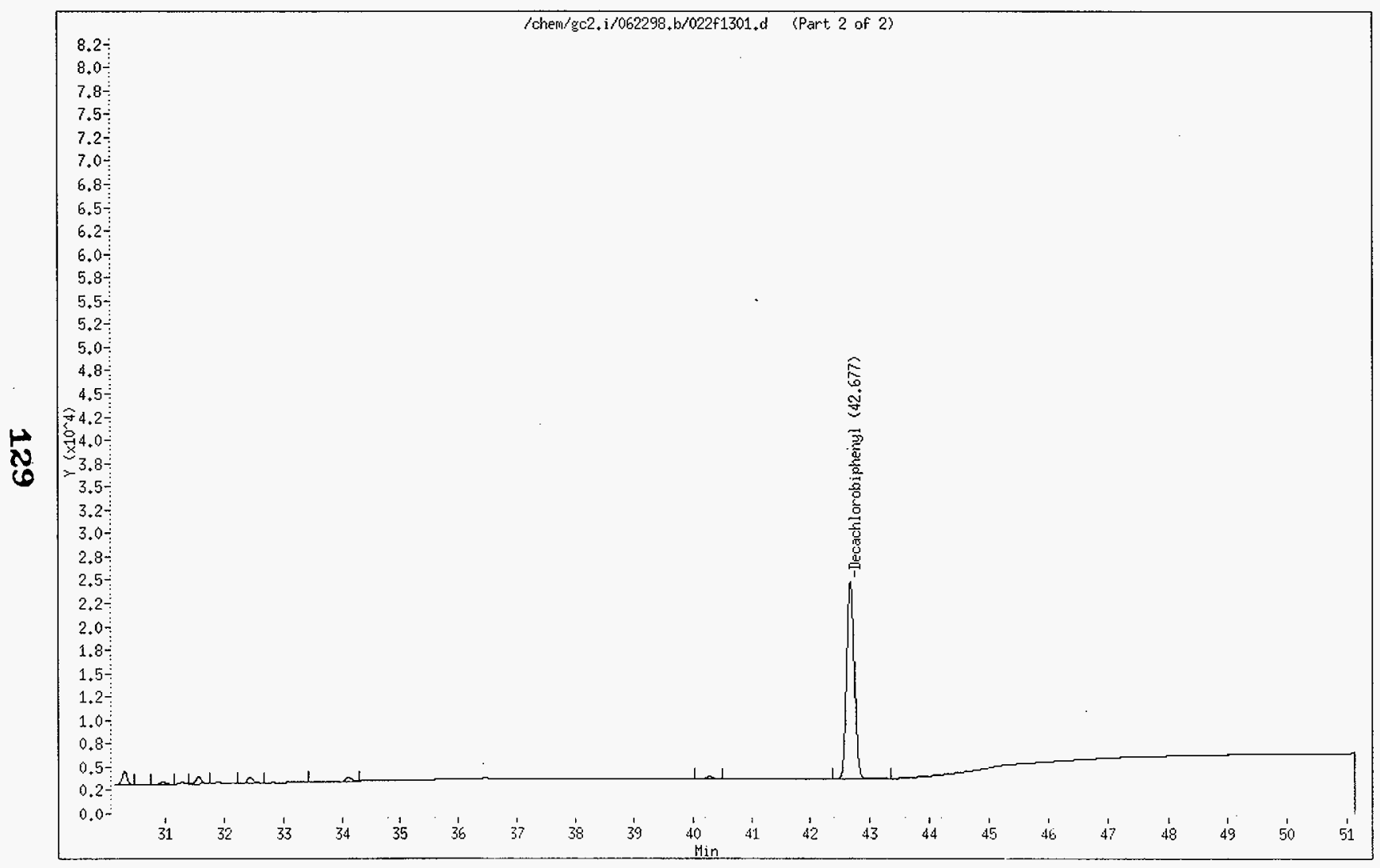


Data File: /chem/gc2.i/062298.b/023f1301.d

Report Date: 20-Jul-1998 06:26

\section{2-S Laboratory}

Data file:/chem/gc2.i/062298.b/023f1301.d

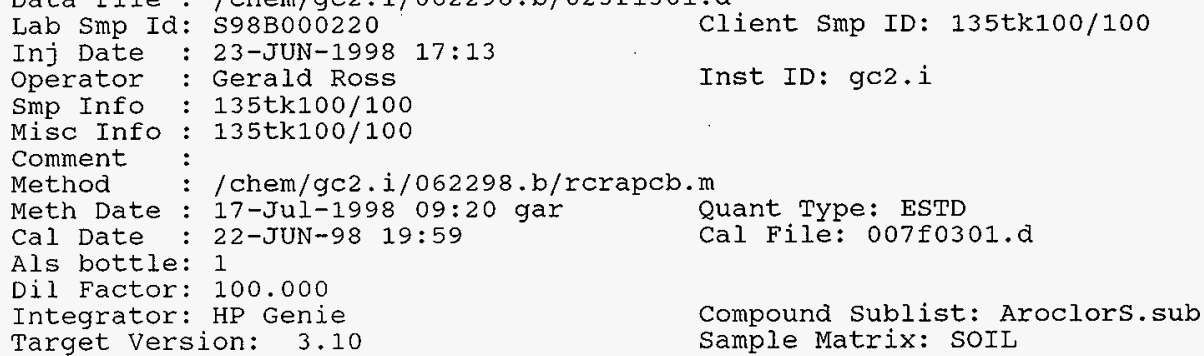

Target Version: 3.10

Sample Matrix: SoIL

\begin{tabular}{|c|c|c|c|c|c|c|}
\hline & & & CONCENTR & TIIONS & & \\
\hline & & & $\mathrm{ON}=\mathrm{COL}$ & FINAL & & \\
\hline RT & EXP RT DLI RT & RESPONSE & ( $n g$ ) & $(u g / K g)$ & TARGE T RANGE & RATIO \\
\hline$==$ & 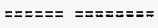 & $=== \pm====$ & $==\pi=\pi==$ & 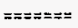 & 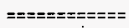 & $=\Xi===$ \\
\hline & clor-1016 & & & CAS \#: & $12674-11-2$ & \\
\hline
\end{tabular}

Operator disabled compound identification.

\begin{tabular}{|c|c|c|c|c|c|c|c|c|}
\hline \multicolumn{4}{|c|}{23 Aroclor -1221} & \multicolumn{5}{|c|}{ CAS \#: $11104-28-2$} \\
\hline 10.800 & 10.811 & -0.011 & 185944 & & & & & 100.00 \\
\hline 11.899 & 11.923 & -0.024 & 43950 & & & $75.00-$ & 115.00 & 23.64 \\
\hline 13.279 & 13.282 & -0.003 & 61456 & 0.312 & 27900 & $208.00-$ & 258.00 & 33.05 \\
\hline 13.628 & 13.673 & -0.045 & 47107 & 0.209 & 18600 & $0.00-$ & 0.00 & 25.33 \\
\hline 13.837 & 13.849 & -0.012 & 166537 & 0.334 & 29800 & $0.00-$ & 0.00 & 89.56 \\
\hline \multicolumn{5}{|c|}{ Average of } & 25400 & & & \\
\hline
\end{tabular}

24 Aroclor -1232

CAS A: $1114-16-5$

Operator disabled compound identification.

25 Aroctor -1242

CAS \#: 53468-21-9

Operator disabled compound ident ification.

26 Aroctor -1248 CAS \#: $12672-29-6$

Operator disabled compound ident ification.

\begin{tabular}{|c|c|c|c|c|c|c|c|c|}
\hline \multicolumn{4}{|c|}{27 Aroclor -1254} & \multicolumn{5}{|c|}{ CAS \#: 11097-69-1 } \\
\hline 21.742 & 21.767 & -0.025 & 71969 & 0.0883 & 7880 & & & 100.00 \\
\hline 22.821 & 22.846 & -0.025 & 102405 & 0.0950 & 8470 & $112.00-$ & 152.00 & 142.29 \\
\hline 24.754 & 24.780 & -0.026 & 97100 & 0.100 & 8950 & $96.00-$ & 136.00 & 134.92 \\
\hline \multicolumn{3}{|c|}{ Average o } & oncentre & ions $=$ & 8430 & & & \\
\hline
\end{tabular}


Data File: /chem/gc2.i/062298.b/023f1301.d

Report Date: 20-Jul-1998 06:26

\begin{tabular}{|c|c|c|c|c|c|c|}
\hline & & & CONCENT & ATIONS & & \\
\hline & & & $\mathrm{ON}-\mathrm{COL}$ & FINAL & & \\
\hline $\mathrm{RT}$ & EXP RT DLT RT & RESPONSE & ( $\mathrm{ng})$ & $(\mathrm{ug} / \mathrm{Kg})$ & TARGET RANGE & RATIO \\
\hline$==$ & 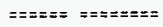 & $==\pi===$ = & $==\Sigma====$ & $==ニ=ニ ニ=$ & 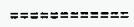 & $=z===$ \\
\hline & roclor -1260 & & & CAS \#: & $13096-82-5$ & \\
\hline
\end{tabular}

operator disabled compound identification.

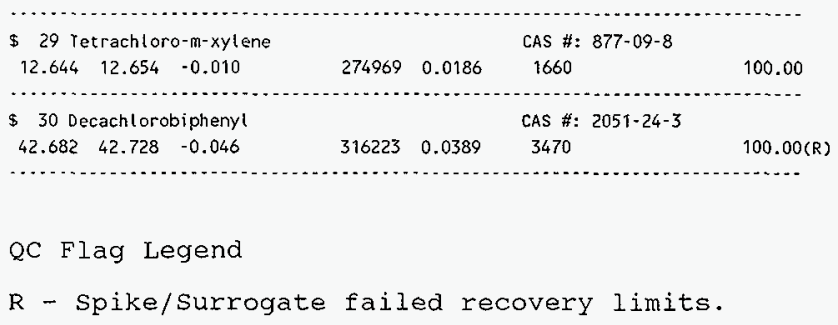


Data File: /chem/gc2.i/062298.b/023f1301.d

Report Date: 20-Jul-1998 06:26

$$
\text { 222-S Laboratory }
$$

RECOVERY REPORT

Client Name:

Sample Matrix: SOLID

Lab Smp Id: S98B000220

Level: LOW

Data Type: GC MULTI COMP

SpikeList File: RESOLUTION.spk

Method File: /chem/gc2.i/062298,b/rcrapcb.m

Misc Info: $135 \mathrm{tk} 100 / 100$

Client SDG: 062298

Fraction: PEST

Client Smp ID: 135tk100/100

Operator: Gerald Ross

sampleType: SAMPLE

Quant Type: ESTD

\begin{tabular}{|c|c|c|c|c|}
\hline SURROGATE COMPOUND & $\begin{array}{l}\text { CONC } \\
\text { ADDED } \\
\mathrm{ug} / \mathrm{Kg}\end{array}$ & $\begin{array}{c}\text { CONC } \\
\text { RECOVERED } \\
\text { ug/Kg }\end{array}$ & RECOVERED & LIMITS \\
\hline $\begin{array}{lll}\$ & 29 & \text { Tetrachloro-m-xyle } \\
\$ & 30 & \text { Decachlorobiphenyl }\end{array}$ & $\begin{array}{l}1780 \\
1780\end{array}$ & $\begin{array}{l}1660 \\
3470\end{array}$ & $\begin{array}{c}93 \cdot 24 \\
194.35 *\end{array}$ & $\begin{array}{l}50-150 \\
50-150\end{array}$ \\
\hline
\end{tabular}


Data File: /chem/gc2.i/062298.b/023f1301.d

Report Date: 20-Jul-1998 06:26

222-S Laboratory

TARGET COMPOUNDS

Client Name:

Lab Smp Id: S98B000220

Sample Location:

Sample Date:

Sample Matrix: SOIL

Analysis Type: PEST

Data Type: GC MULTI COMP

Misc Info: $135 \mathrm{tk} 100 / 100$
Client SDG: 062298

Client Smp ID: 135tk100/100

Sample Point:

Date Received:

Quant Type: ESTD

Level: LoW

Operator: Gerald Ross

CONCENTRATION UNITS:

$(\mathrm{ug} / \mathrm{L}$ or $\mathrm{ug} / \mathrm{KG}) \mathrm{ug} / \mathrm{Kg}$
$Q$

\begin{tabular}{r|c|}
3300 & $\mathrm{U}$ \\
25400 & \\
3300 & $\mathrm{U}$ \\
3300 & $\mathrm{U}$ \\
3300 & $\mathrm{U}$ \\
8430 & \\
3300 & $\mathrm{U}$ \\
$===========$ \\
1660 \\
3470
\end{tabular} \mid$===$


Sample Info: 135tk100/100

Volume Injected (uL): 1.0

Dperator: Gerald Ross

Column phase: $x t i-5$

Columin dianeter: 0.25

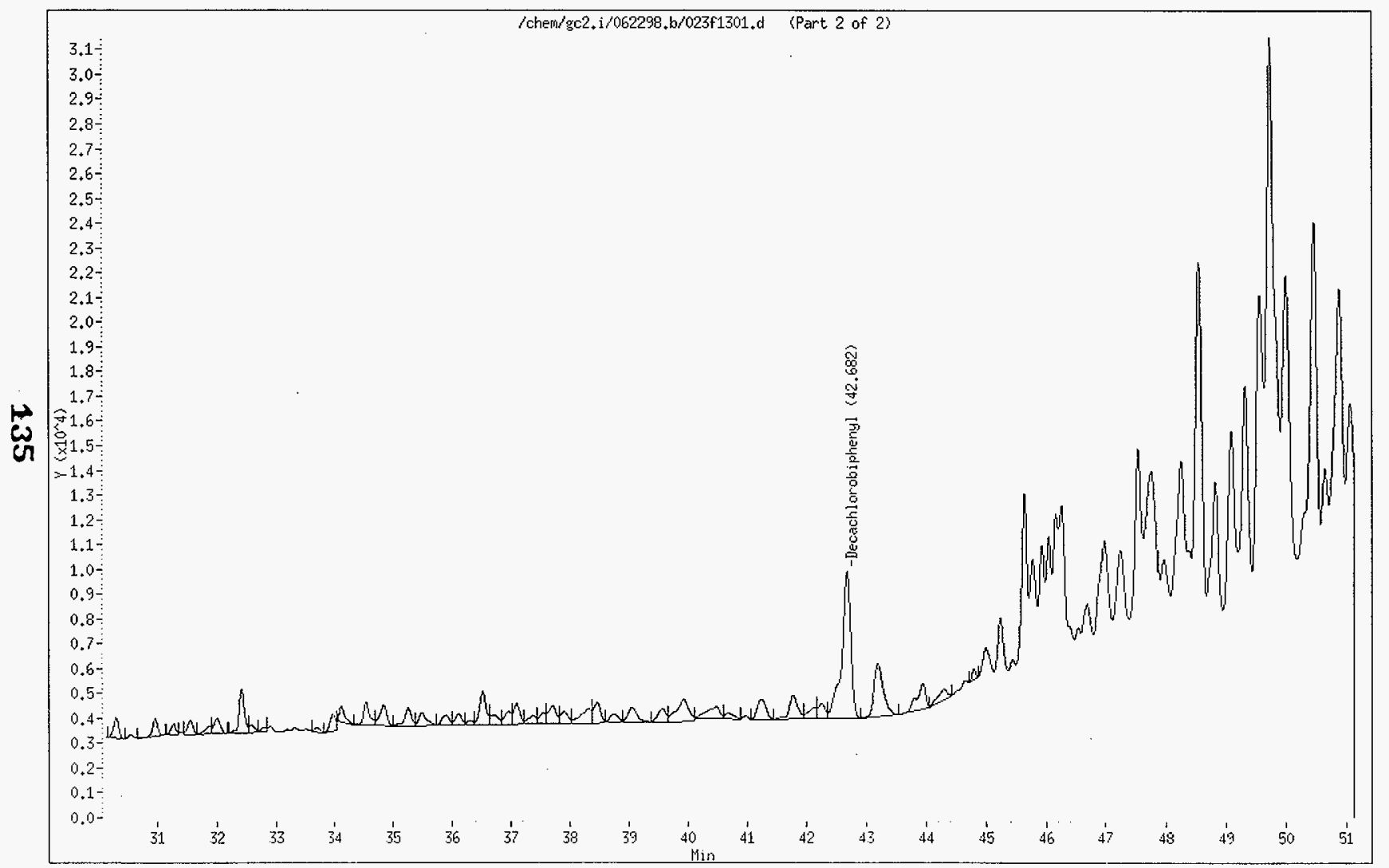


Data File: /chem/gc2.i/062298.b/025f1301.d Report Date: 20-Jul-1998 07:30

\section{2-s Laboratory}

Data file:/chem/gc2.i/062298.b/025f1301.d

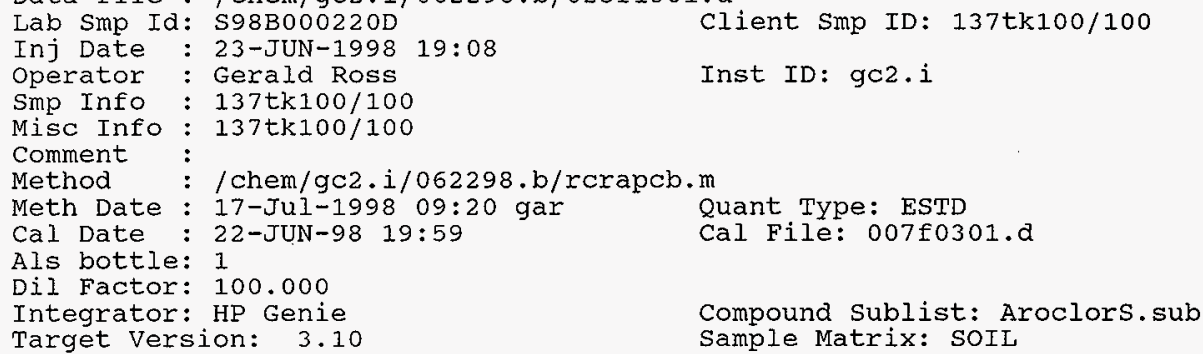

\begin{tabular}{|c|c|c|c|c|c|c|}
\hline \multirow[b]{3}{*}{$R T$} & \multirow[b]{3}{*}{ EXP RT DLT RT } & & \multicolumn{2}{|c|}{ CONCENTRATIONS } & \multirow[b]{3}{*}{ TARGET RANGE } & \multirow[b]{3}{*}{ RATIO } \\
\hline & & & $\mathrm{ON}-\mathrm{COL}$ & FINAL & & \\
\hline & & RESPONSE & ( $\mathrm{ng})$ & (ug/Kg) & & \\
\hline$==$ & 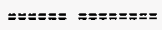 & $========$ & $======+$ & $======$ & $======== \pm==8$ & $====$ \\
\hline & oclor-1016 & & & CAS \# & $12674-11-2$ & \\
\hline
\end{tabular}

Operator disabled compound identification.

\begin{tabular}{|c|c|c|c|c|c|c|c|c|}
\hline \multicolumn{4}{|c|}{23 Aroclor $=1223$} & \multicolumn{5}{|c|}{ CAS \#: $11104-28-2$} \\
\hline 10.802 & 10.811 & -0.009 & 94238 & & & & & $100.00(\mathrm{M}$ \\
\hline 11.906 & 11.923 & -0.017 & 20456 & & & $75.00-$ & 115.00 & 21.71 \\
\hline 13.283 & 13.282 & 0.001 & 43436 & 0.221 & 17300 & $208.00-$ & 258.00 & 46.09 \\
\hline 13.630 & 13.673 & -0.043 & 31098 & 0.138 & 10800 & 0.00 & 0.00 & 33.00 \\
\hline 13.840 & 13.849 & -0.009 & 112258 & 0.225 & 17600 & $0.00-$ & 0.00 & 119.12 \\
\hline \multicolumn{5}{|c|}{ Average of Peak } & 15300 & & & \\
\hline
\end{tabular}

24 Aroclor -1232

CAS \#: $1114-16-5$

Operator disabled compound identification.

25 Aroclor -1242

CAS \#: 53469-21-9

Operator disabled compound identification.

26 Aroclor -1248

CAS \#: $12672-29-6$

Operator disabled compound identification.

\begin{tabular}{|c|c|c|c|c|c|c|c|c|}
\hline \multicolumn{4}{|c|}{27 Aroclor -1254} & \multicolumn{5}{|c|}{ CAS \#: $11097-69-1$} \\
\hline 21.742 & 21.767 & -0.025 & 66506 & 0.0816 & 6400 & & & 100.00 \\
\hline 22.822 & 22.846 & -0.024 & 97930 & 0.0908 & 7120 & $112.00-$ & 152.00 & 147.25 \\
\hline 24.755 & 24.780 & -0.025 & 90709 & 0.0938 & 7360 & $96.00-$ & 136.00 & 136.39 \\
\hline \multicolumn{5}{|c|}{ Average of Pea } & 6960 & & & \\
\hline
\end{tabular}


Data File: /chem/gc2,i/062298.b/025f1301.d

Report Date: 20-Jul-1998 07:30

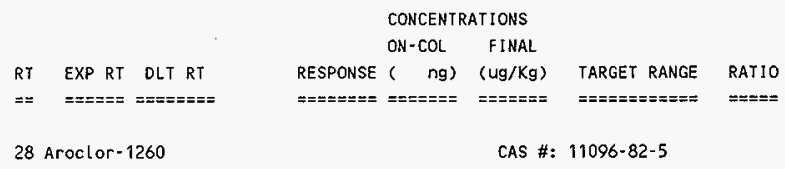

Operator disabled compound identification.

$\$ 29$ Tetrachloro-m-xylene

CAS \#: $877-09-8$

$\begin{array}{lll}12.644 & 12.654 & -0.010\end{array}$

1690

100.00

\$ 30 Decachlorobiphenyl

CAS \#: $2051-24-3$

$42.682 \quad 42.728 \quad-0.046$

2740

$100.00(R)$

QC Flag Legend

R - Spike/Surrogate failed recovery limits.

M - Compound response manually integrated. 
Data File: /chem/gc2.i/062298.b/025f1301.d

Report Date: 20-Jul-1998 07:30

\section{2-S Laboratory}

RECOVERY REPORT

Client Name:

Sample Matrix: SOLID

Client SDG: 062298

Lab Smp Id: S98B000220D

Fraction: PEST

Level: LoW

Data Type: GC MULTI COMP

SpikeList File: RESOLUTION.spk

Method File: /chem/gc2.i/062298.b/rcrapcb.m

Misc Info: $137 \mathrm{tk} 100 / 100$

\begin{tabular}{|c|c|c|c|c|}
\hline SURROGATE COMPOUND & $\begin{array}{l}\text { CONC } \\
\text { ADDED } \\
\mathrm{ug} / \mathrm{Kg}\end{array}$ & $\begin{array}{c}\text { CONC } \\
\text { RECOVERED } \\
\text { ug./Kg }\end{array}$ & RECOVERED & LIMITS \\
\hline$\$ 29$ Tetrachloro-m-xyle & 1570 & 1690 & 107.48 & $\overline{50-150}$ \\
\hline$\$ 30$ Decachlorobiphenyl & 1570 & 2740 & $174.92 *$ & $50-150$ \\
\hline
\end{tabular}


Data File: /chem/gc2.i/062298.b/025f1301.d

Report Date: 20-Jul-1998 07:30

\section{2-S Laboratory}

TARGET COMPOUNDS

Client Name:

Lab Smp Id: S98B000220D

Sample Location:

Sample Date:

Sample Matrix: SOIL Analysis Type: PEST

Data Type: GC MULTI COMP

Misc Info: $137 \mathrm{tk} 100 / 100$
Client SDG: 062298

client Smp ID: 137tk100/100

Sample Point:

Date Received:

Quant Type: ESTD

Level: LOW

Operator: Gerald Ross

CONCENTRATION UNITS:

(ug/L or ug/KG) ug/Kg

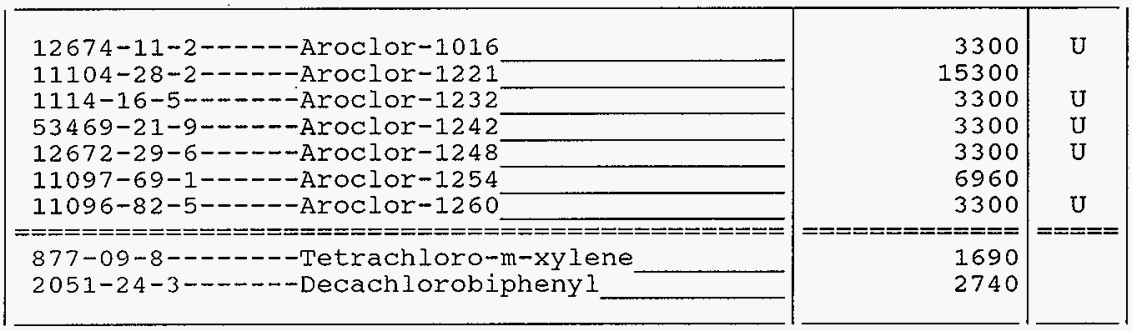


23-JuN -1998 19:08

Client II: $137 \mathrm{tk} 100 / 100$

Sample Info: 137tk100/100

Volume Injected $\{u L\}: 1.0$

Operator: Gerald Ross

Column phase: $x t i-5$

Column diameter: 0.25

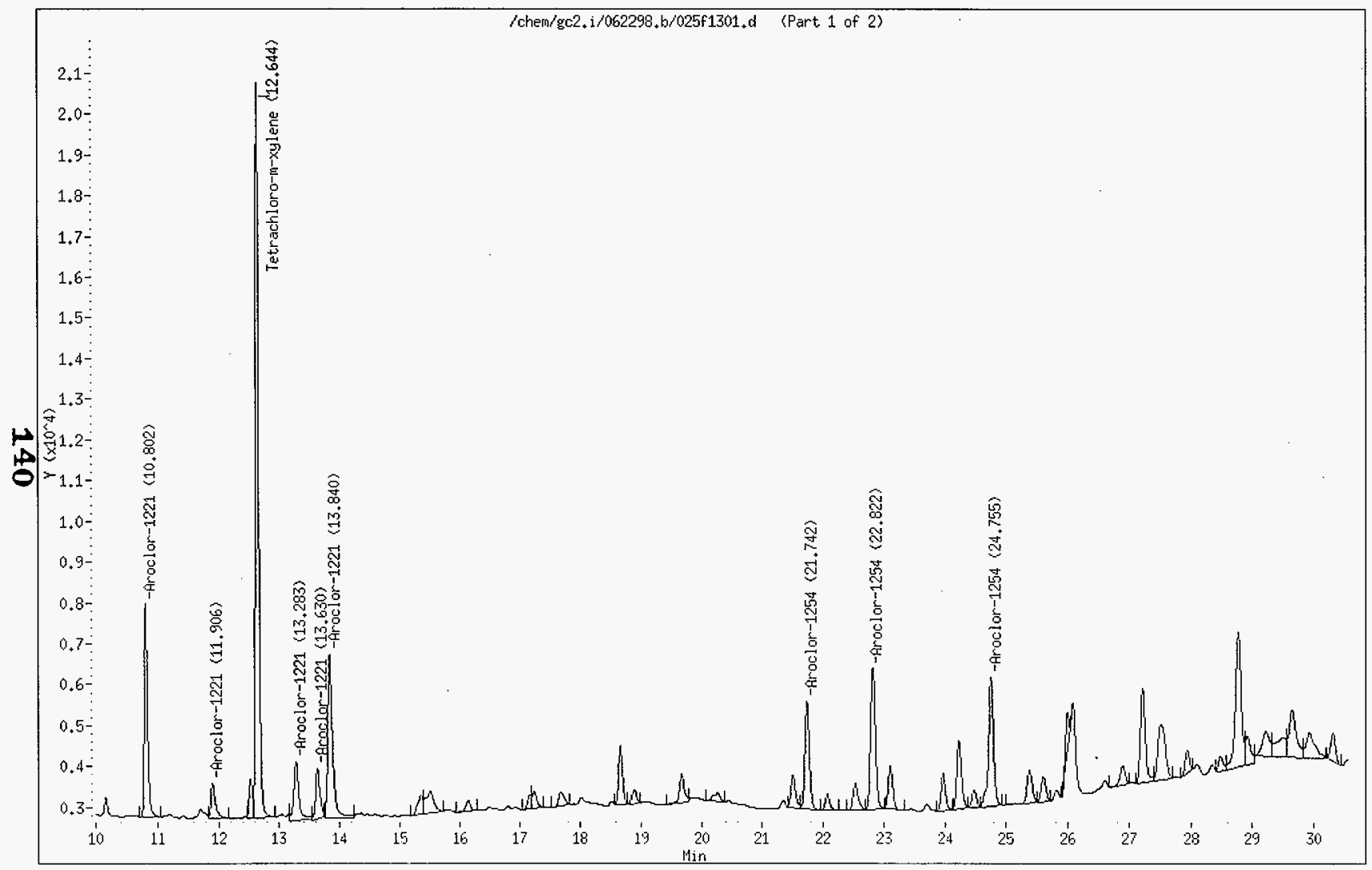


HNF-SD-WM-DP-309 REV. 0

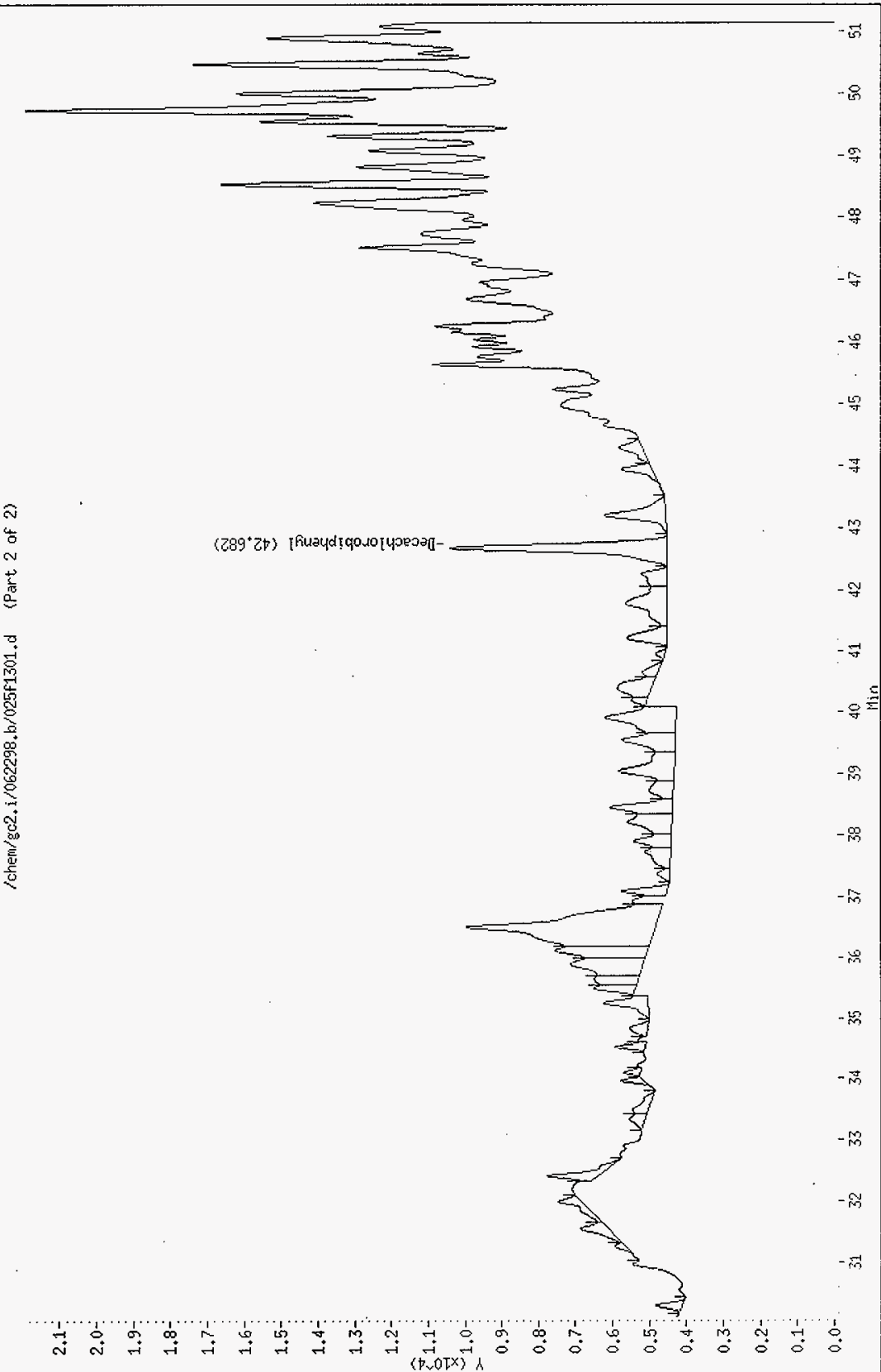


Data File: /chem/gc2.i/062298.b/027f1301.d

Report Date: 20-Jul-1998 06:26

\section{2-S Laboratory}

Data file : /chem/gc2.i/062298.b/027f1301.d

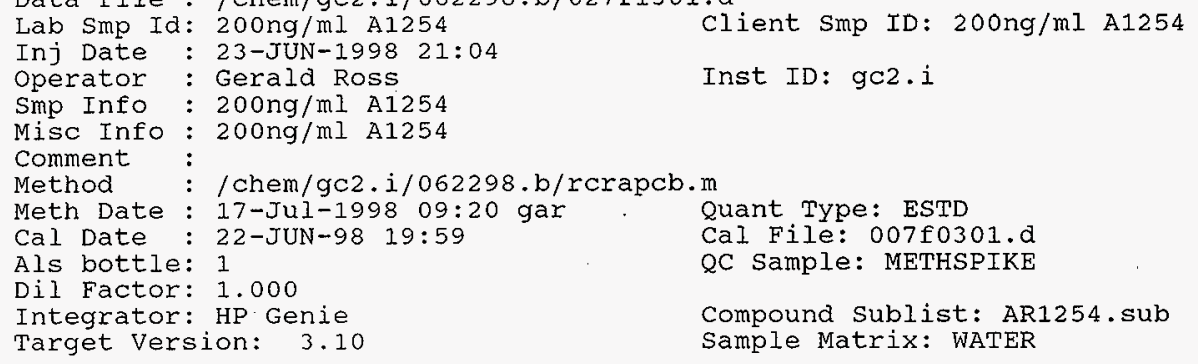
Target Version: $3.10 \quad$ Sample Matrix: WATER

Client Smp ID: $200 \mathrm{ng} / \mathrm{ml}$ A1254 Inst ID: $\mathrm{gc} 2 . \mathrm{i}$ QC Sample: METHSPIKE

Compound Sublist: AR1254.sub

\begin{tabular}{|c|c|c|c|c|c|c|c|}
\hline & & & & CONCENTR & ATIONS & & \\
\hline & & & & $\mathrm{ON}-\mathrm{COL}$ & FINAL & & \\
\hline$R T$ & EXP RT & DLI RT & RESPONSE & ( $\mathrm{ng})$ & $(\mathrm{ug} / L)$ & TARGET RANGE & RATIO \\
\hline$==$ & $z= \pm x==$ & 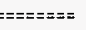 & $==\Sigma=ニ=ニ=$ & $===ニ=ニ=$ & $=======$ & $============$ & $=====$ \\
\hline $27 \mathrm{Ar}$ & roclor-1 & 254 & & & CAS \#: & : $11097-69-1$ & \\
\hline 21.740 & 21.767 & -0.027 & 163253 & 0.200 & 4.00 & & 100.00 \\
\hline 22.820 & 22.846 & -0.026 & 211562 & 0.196 & 3.92 & $112.00-152: 00$ & 129.59 \\
\hline 24.753 & 24.780 & -0.027 & 196065 & 0.203 & 4.05 & $96.00-136.00$ & 120.10 \\
\hline & & Average 0 & Concentre & ations = & 3.99 & & \\
\hline$\$ 29 \mathrm{r}$ & etrachlo & ro-m=xyle & & & CAS \#: & : $877-09-8$ & \\
\hline 12.641 & 12.654 & -0.013 & 1351424 & 0.0916 & 1.83 & & 100.00 \\
\hline$\$ 300$ & ecachlor & obiphenyl & & & CAS \#: & $=2051-24-3$ & \\
\hline 42.679 & 42.728 & -0.049 & 800141 & 0.0984 & 1.97 & & 100.00 \\
\hline
\end{tabular}


Data File: /chem/gc2.i/062298.b/027f1301.d Report Date: 20-Jul-1998 06:26

\section{2-S Laboratory \\ RECOVERY REPORT}

Client Name:

Sample Matrix: LIQUID

Lab Smp Id: $200 \mathrm{ng} / \mathrm{ml}$ A1254

Level: LoW

Data Type: GC MULTI COMP

Spikelist File: Aroclor-1254.spk

Method File:/chem/gc2.i/062298.b/rcrapcb.m

Client SDG: 062298

Fraction: PEST

Client Smp ID: 200ng/ml A1254

operator: Gerald Ross

SampleType: METHSPIKE

Quant Type: ESTD

Misc Info: $200 \mathrm{ng} / \mathrm{ml}$ A1254

\begin{tabular}{|c|c|c|c|c|}
\hline SPIKE COMPOUND & $\begin{array}{l}\text { CONC } \\
\text { ADDED } \\
\text { ug/L }\end{array}$ & $\begin{array}{c}\text { CONC } \\
\text { RECOVERED } \\
\text { ug/L }\end{array}$ & RECOVERED & LIMITS \\
\hline 27 Aroclor-1254 & 4.00 & 3.99 & 99.85 & $\overline{50-150}$ \\
\hline SURROGATE COMPOUND & $\begin{array}{l}\text { CONC } \\
\text { ADDED } \\
\mathrm{ug} / \mathrm{L}\end{array}$ & $\begin{array}{c}\text { CONC } \\
\text { RECOVERED } \\
\text { Ug/L }\end{array}$ & $\begin{array}{c}\% \\
\text { RECOVERED }\end{array}$ & LIMITS \\
\hline $\begin{array}{lll}\$ & 29 & \text { Tetrachloro-m-xyle } \\
\$ & 30 & \text { Decachlorobiphenyl }\end{array}$ & $\begin{array}{l}2.00 \\
2.00\end{array}$ & $\begin{array}{l}1.83 \\
1.97\end{array}$ & $\begin{array}{l}91.65 \\
98.35\end{array}$ & $\begin{array}{l}50-150 \\
50-150\end{array}$ \\
\hline
\end{tabular}


Data File: /chem/gc2.i/062298.b/027f1301.d

Report Date: 20-Jul-1998 06:26

$$
\begin{aligned}
& \text { 222-S Laboratory } \\
& \text { TARGET COMPOUNDS }
\end{aligned}
$$

Client Name:

Lab Smp Id: $200 \mathrm{ng} / \mathrm{ml}$ A1254

Sample Location:

Sample Date:

Sample Matrix: WATER

Analysis Type: PEST

Data Type: GC MULTI COMP

Misc Info: 200ng/ml A.1254
Client SDG: 062298

Client Smp ID: $200 \mathrm{ng} / \mathrm{ml}$ A1254

Sample Point:

Date Received:

Quant Type: ESTD

Level: LoW

Operator: Gerald Ross

CONCENTRATION UNITS:

( $\mathrm{ug} / \mathrm{L}$ or $\mathrm{ug} / \mathrm{KG}$ ) ug/L

\begin{tabular}{|c|c|c|}
\hline $11097-69-1-----$ Aroclor -1254 & 3.99 & \\
\hline 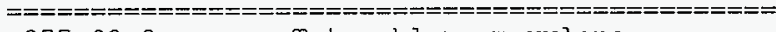 & $============$ & $====$ \\
\hline 877-09-8-1.m-Tetrachloro-m-xylene & 1.83 & \\
\hline $205 \perp-24-3-------$ decacnior 001 pneny 1 & & \\
\hline
\end{tabular}


Client ID: 200 ong $/ \mathrm{ml}$ A1254

Instrument: $\mathrm{gc} 2 . \mathrm{i}$

Sample Info: $200 \mathrm{ng} / \mathrm{ml}$ A1254

Volume Injected $\langle u L\rangle: 1,0$

Operator: Gerald Ross

Column phase: $\mathrm{Xti}-5$

Columin di aneter: 0.25

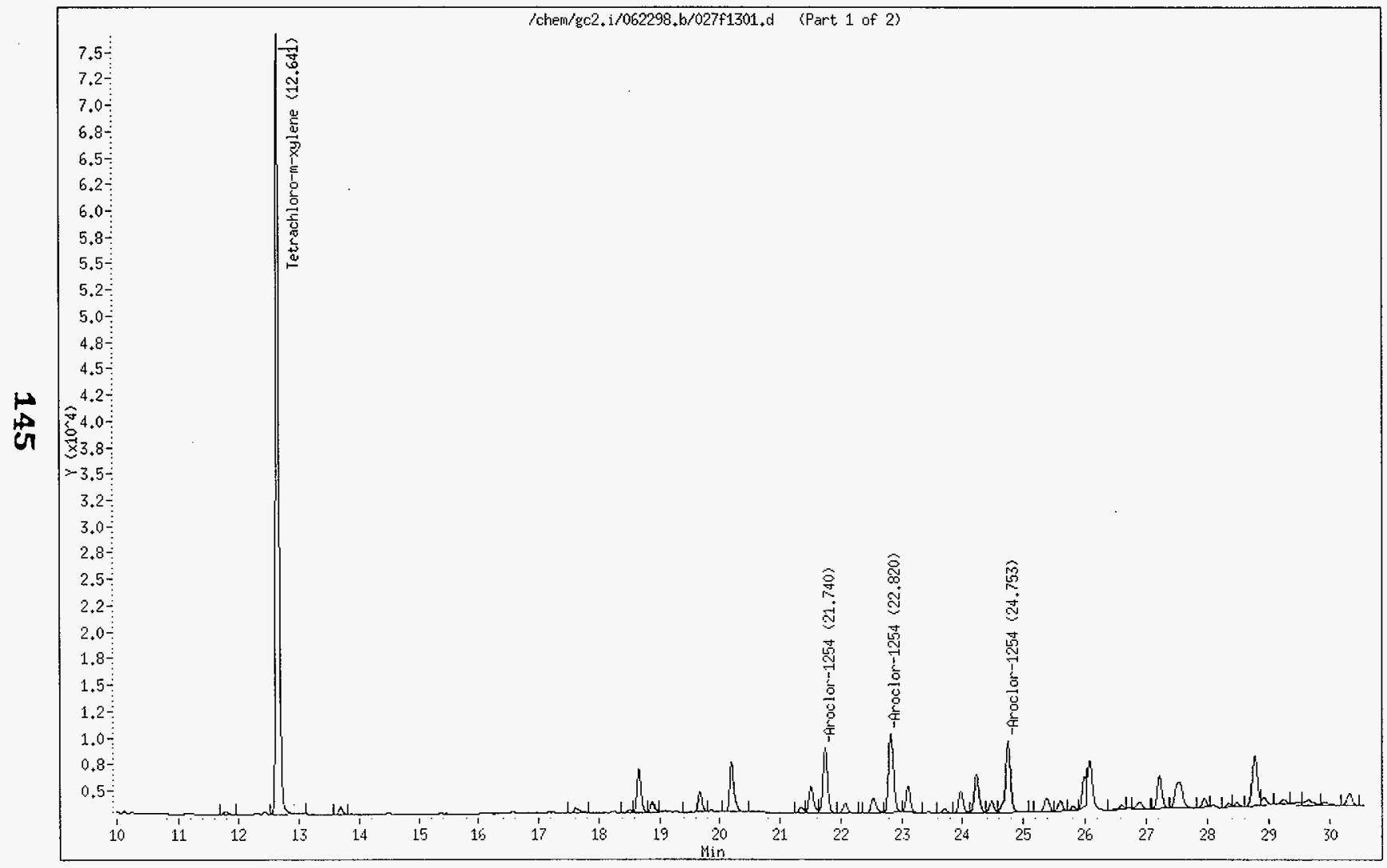


ate : 23-JUN-1998 21:04

Client ID: 200ng/ml R1254

Sample Info: 200ng/ml A1254

Volume Injected $\{u L\}: 1.0$

Dperator: Gerald Ross

Column phase: $x_{t} \mathbf{i}-5$

Colums diameter: 0.25

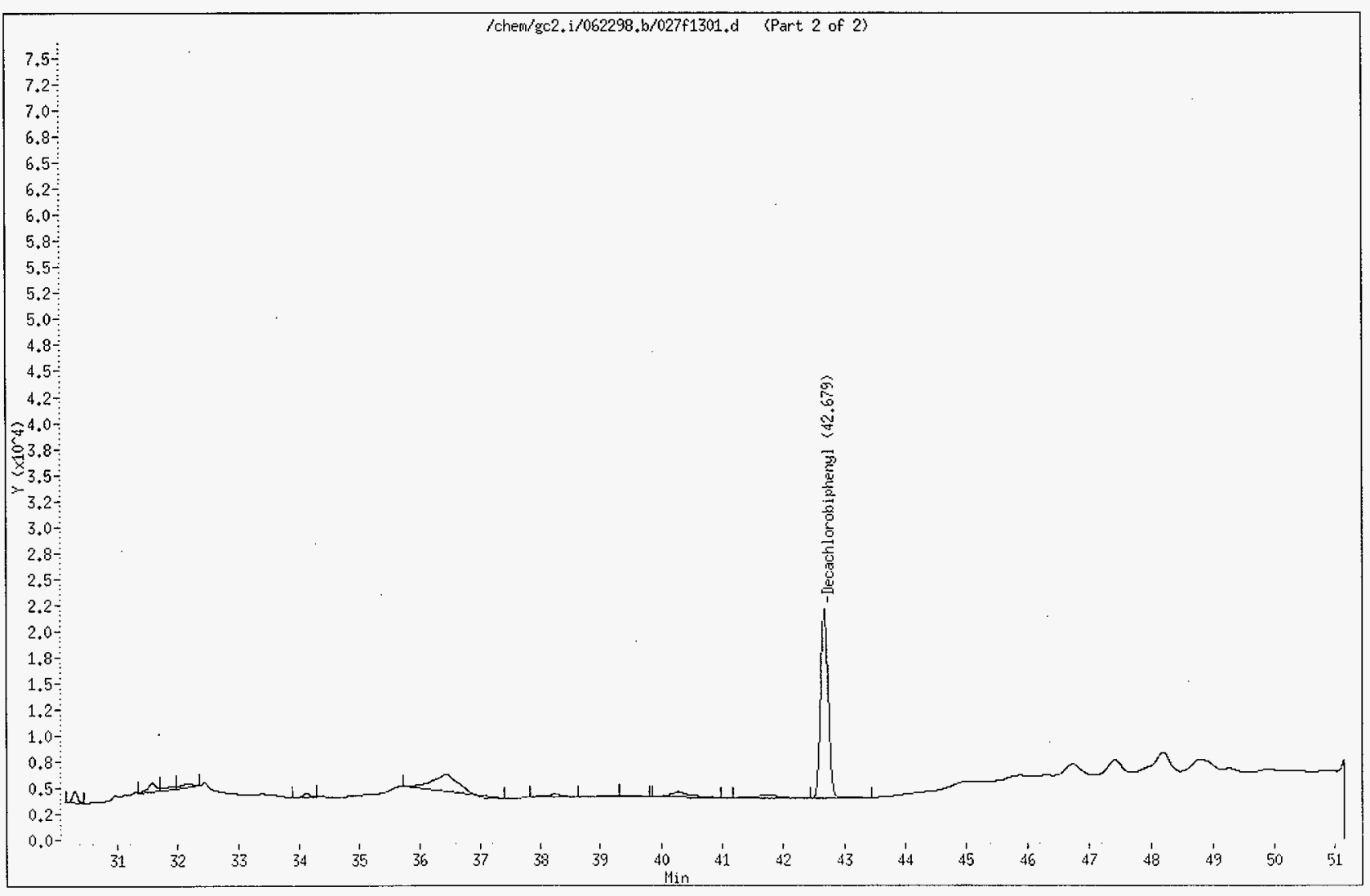

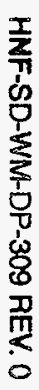


Data File: /chem/gc2.i/062298.b/027fl401.d

Report Date: 29-Jun-1998 13:42

\section{2-S Laboratory}

Data file:/chem/gc2.i/062298.b/027f1401.d

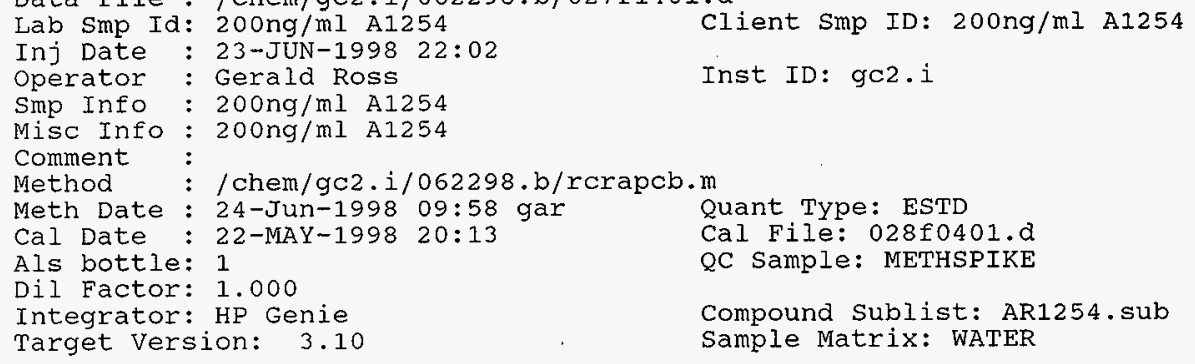

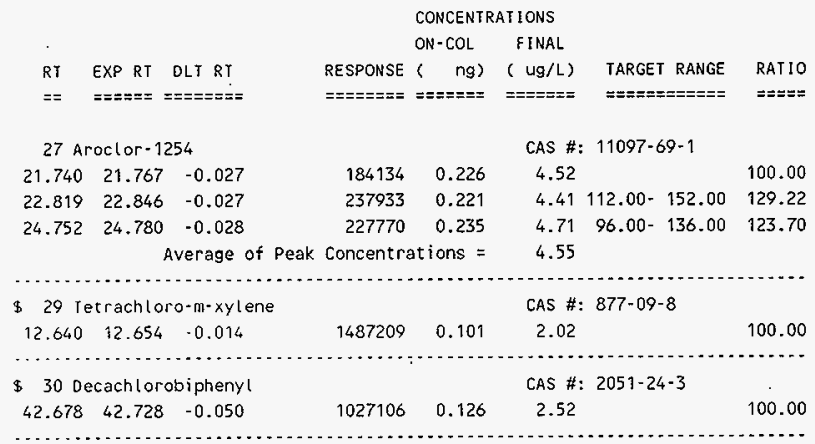


Data File: /chem/gc2.i/062298.b/027f1401.d

Report Date: 29-Jun-1998 13:42

\section{2-S Laboratory}

RECOVERY REPORT

Client Name:

Sample Matrix: LIQUID

Lab Smp Id: $200 \mathrm{ng} / \mathrm{ml}$ A1254

Leve 1: LoW

Data Type: GC MULTI COMP

Spikelist File: Aroclor $-1254.5 p k$

Method File:/chem/gc2.i/062298.b/rcrapcb.m

Client SDG: 062298

Fraction: PEST

Client Smp ID: 200ng/ml A1254

Operator: Gerald Ross

SampleType: METHSPIKE

Quant Type: ESTD

Misc Info: 200ng/mI A1254

\begin{tabular}{|c|c|c|c|c|}
\hline SPIKE COMPOUND & $\begin{array}{l}\text { CONC } \\
\text { ADDED } \\
\text { ug/I }\end{array}$ & $\begin{array}{c}\text { CONC } \\
\text { RECOVERED } \\
\text { ug/L }\end{array}$ & RECOVERED \\
\hline 27 Aroclor-1254 & $\frac{4.00}{4.55}$ & $\frac{1}{113.65}$ & $\frac{50-150}{50}$ \\
\hline
\end{tabular}

\begin{tabular}{|c|c|c|c|c|}
\hline SURROGATE COMPOUND & $\begin{array}{l}\text { CONC } \\
\text { ADDED } \\
\text { uq } / L\end{array}$ & $\begin{array}{c}\text { CONC } \\
\text { RECOVERED } \\
\text { UG } / \mathrm{L}\end{array}$ & RECOVERED & LIMITS \\
\hline $\begin{array}{lll}\$ & 29 & \text { Tetrachloro-m-xyle } \\
\$ & 30 & \text { Decachlorobiphenyl }\end{array}$ & $\begin{array}{l}2.00 \\
2.00\end{array}$ & $\begin{array}{l}2.02 \\
2.52\end{array}$ & $\begin{array}{l}100.86 \\
126.25\end{array}$ & $\begin{array}{l}50-150 \\
50-150\end{array}$ \\
\hline
\end{tabular}


Data File: /chem/gc2.i/062298.b/027f1401.d

Report Date: 29-Jun-1998 13:42

\section{2-S Laboratory \\ TARGET COMPOUNDS}

Client Name:

Lab Smp Id: 200ng/ml A1254

Sample Location:

Sample Date:

Sample Matrix: WATER

Analys is Type: PEST

Data Type: GC MULTI COMP

Misc Info: $200 \mathrm{ng} / \mathrm{ml}$ A1254
Client SDG: 062298

Client Smp ID: $200 \mathrm{ng} / \mathrm{ml}$ A1254

Sample Point:

Date Received:

Quant Type: ESTD

Level: LoW

Operator: Gerald Ross

CAS NO.

COMPOUND

CONCENTRATION UNITS:

(ug/L or ug/KG) ug/L

Q

4.55

11097-69-1------Aroclor-1254

$\left|========\begin{array}{r}4.55 \\ =-3.02 \\ 2.52\end{array}\right|=====$


Date : 23-JUN-1998 22:02

Client ID: $200 \mathrm{ng} / \mathrm{ml}$ A1254

Instrument: gc2, i

Sample Info: 200ng/ml A1254

Yolume Injected $(u L): 1.0$

Operator: Gerald Ross

Coluon phase: Xti -5

Column dianteter: 0.25
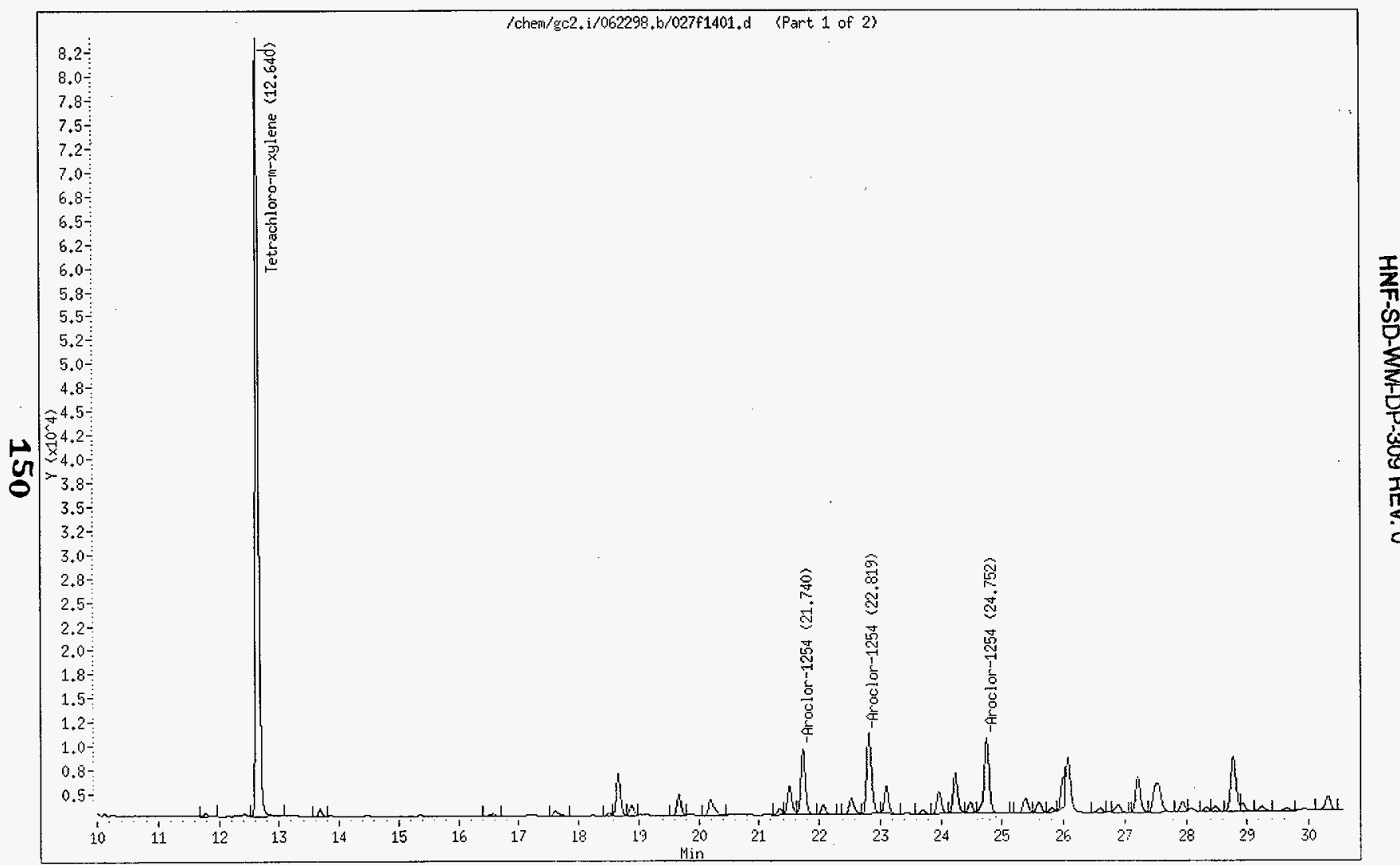
Data File: /chem/gc2, i/062298,b/027f1401,d

Date : 23-JuN-1998 22:02

Client ID: $200 \mathrm{ng} / \mathrm{ml}$ A1254

Sample Info: 200ng/ml A1254

Volume Injected (uL) $\div 1,0$

Instrument: $\mathrm{gc} 2.1$

Column phase: $X_{t i-5}$

Dperator: Gerald Ross

Column diameter: 0.25

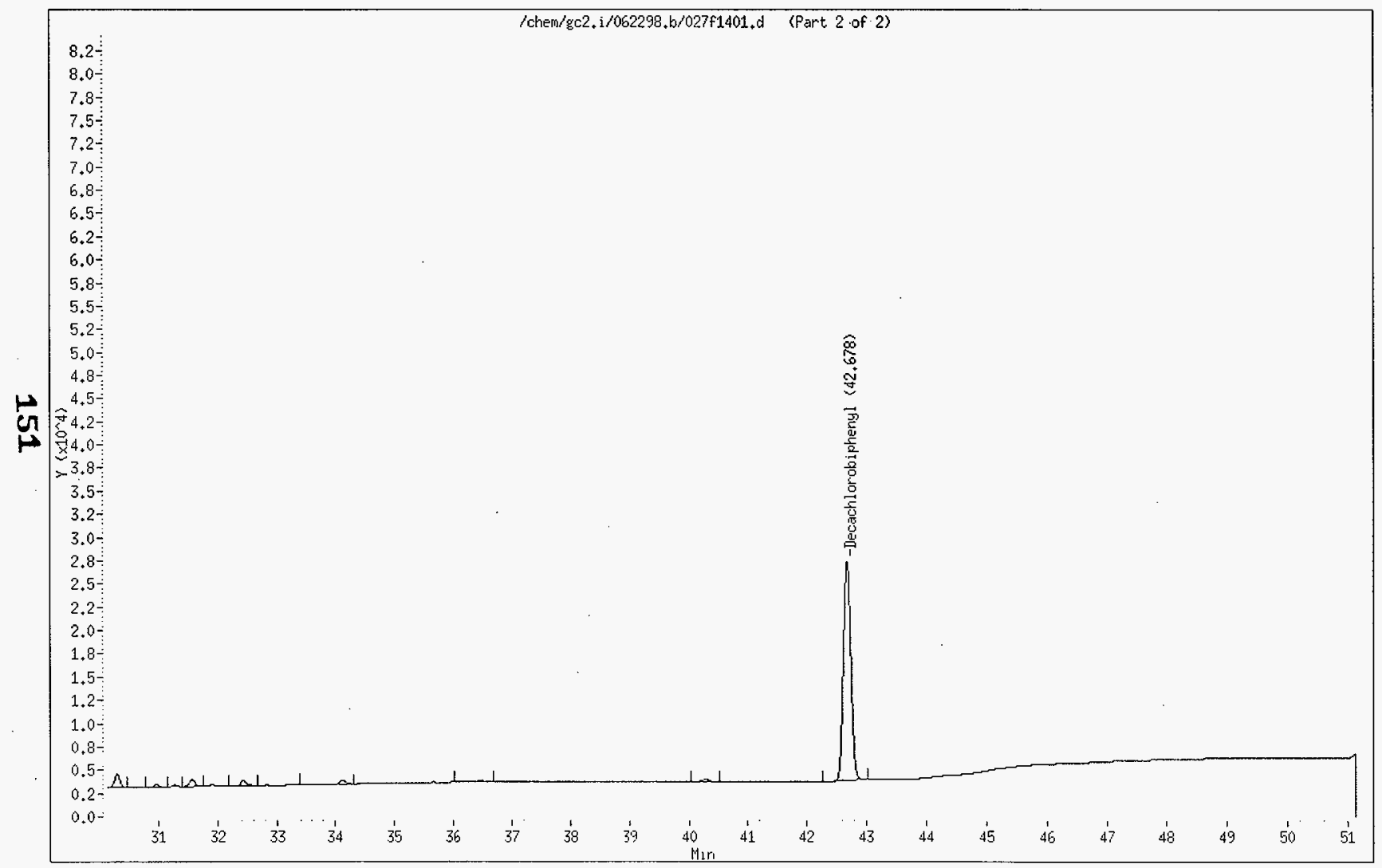


Report Date: 13-Jul-1998 14:37

\section{2-S Laboratory}

Data file:/chem/gc2.i/062298.b/028f1401.d

Lab smp Id: $400 \mathrm{ng} / \mathrm{ml}$ A1221

Inj Date : 23-JUN-1998 23:00

operator : Gerald Ross

Smp Info : 400ng/ml Al221

Misc Info : 400 ng/ml A1221

comment :

Method : /chem/gc2.i/062298.b/rcrapcb.m

Meth Date : 24-Jun-1998 09:58 gar

Cal Date : 22-JUN-98 19:59

Als bottle: 1

Dil Factor: 1.000

Integrator: HP Genie

Target Version: 3.10
Client Smp ID: $400 \mathrm{ng} / \mathrm{ml}$ A1221

Inst ID: $g c 2 . i$
Quant TYpe: ESTD

Cal File: 007f0301.d

Compound Sublist: AR1221.sub Sample Matrix: WATER

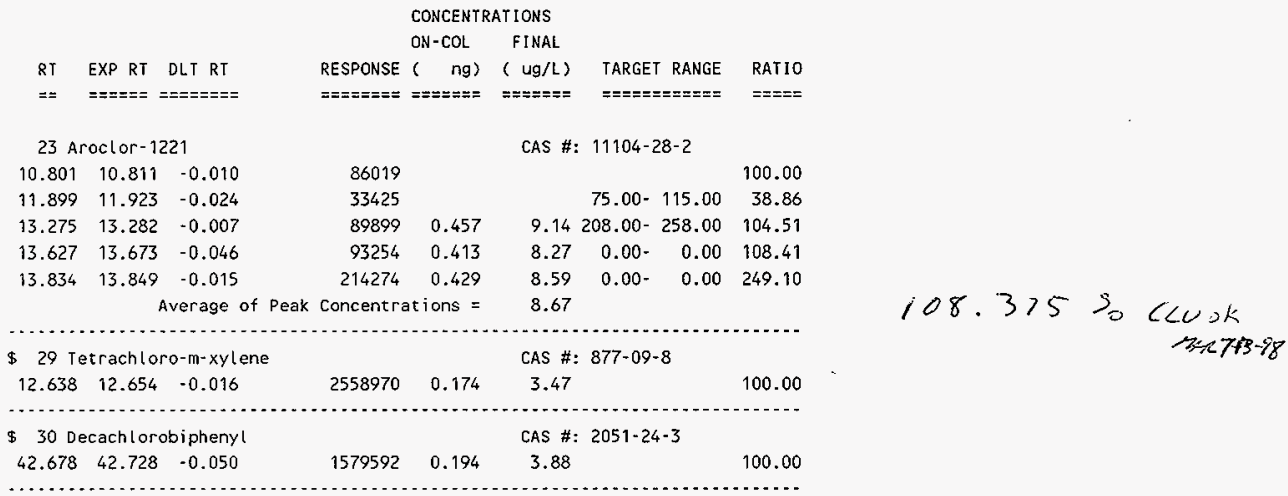


Data File: /chem/gc2.i/062298.b/028f1401.d

Report Date: 13-Jul-1998 14:37

222-S Laboratory

RECOVERY REPORT

Client Name:

Sample Matrix: IIQUID

Lab Smp Id: 400ng/ml Al221

Level: LoW

Data Type: GC MULTI COMP

SpikeList File: Aroclor-1221.spk

Method File: /chem/gc2.i/062298.b/rcrapcb.m

Misc Info: $400 \mathrm{ng} / \mathrm{ml}$ A1221

Client SDG:' 062298

Fraction: PEST

Client Smp ID: 400ng/ml A1221

Operator: Gerald Ross

SampleType: SAMPLE

Quant Type: ESTD

\begin{tabular}{|c|c|c|c|c|}
\hline SURROGATE COMPOUND & $\begin{array}{l}\text { CONC } \\
\text { ADDED } \\
u g / L\end{array}$ & $\begin{array}{c}\text { CONC } \\
\text { RECOVERED } \\
\text { ug/L }\end{array}$ & $\stackrel{\stackrel{\circ}{\circ}}{\text { RECOVERED }}$ & LIMITS \\
\hline $\begin{array}{lrl}\$ & 29 & \text { Tetrachloro-m-xyle } \\
\text { \$ } 30 \text { Decachlorobiphenyl }\end{array}$ & $\begin{array}{l}4.00 \\
4.00\end{array}$ & $\begin{array}{l}3.47 \\
3.88\end{array}$ & $\begin{array}{l}86.77 \\
97.08\end{array}$ & $\begin{array}{l}50-150 \\
50-150\end{array}$ \\
\hline
\end{tabular}


Data File: /chem/gc2.i/062298.b/028f1401.d

Report Date: 13-Jul-1998 14:37

$$
\text { 222-s Laboratory }
$$

TARGET COMPOUNDS

Client Name:

Lab smp Id: 400ng/mI A1221

Sample Location:

Sample Date:

Sample Matrix: WATER

Analysis Type: PEST

Data Type: GC MULTI COMP

Misc Info: $400 \mathrm{ng} / \mathrm{ml} \mathrm{A} 1221$
Client SDG: 062298

Client Smp ID: $400 \mathrm{ng} / \mathrm{ml}$ A1221

Sample point:

Date Received:

Quant Type: ESTD

Level: LoW

operator: Gerald Ross

CONCENTRATION UNITS:

( $\mathrm{ug} / \mathrm{L}$ or $\mathrm{ug} / \mathrm{KG}$ ) ug/L

\begin{tabular}{|c|c|c|}
\hline $11104-28-2---m-$ Aroclor -1221 & $\begin{array}{r}8.67 \\
\end{array}$ & \\
\hline 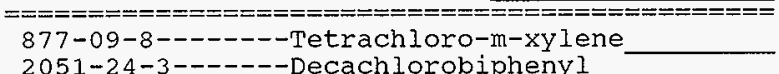 & $\begin{aligned}=-m=== & === \\
& 3.47 \\
& 3.88\end{aligned}$ & $====$ \\
\hline
\end{tabular}


Sample Info: d00ng/ml A1221

Volume Injected $(u L): 1,0$

Column phase: $\mathrm{Xti}_{\mathrm{t}-5}$

Operator: Gerald Ross

Column diameter: 0,25

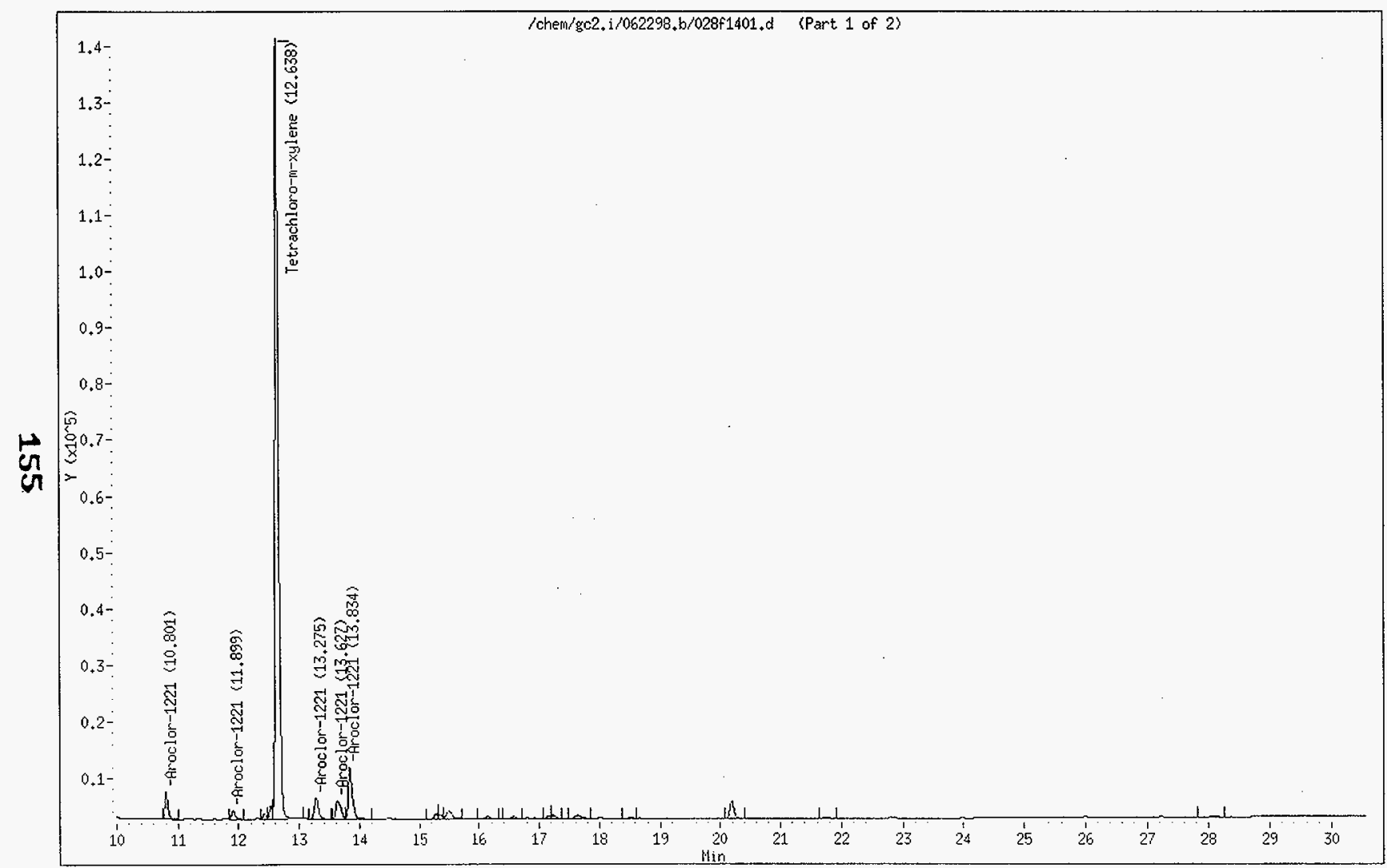


Data File: /chem/gc2.i/062298.b/029f1401.d Report Date: 29-Jun-1998 13:43

\section{2-S Laboratory}

Data file : /chem/gc2.i/062298.b/029f1401.d
Lab Smp Id: $200 \mathrm{ng} / \mathrm{ml}$ A1660
Inj Date : 23-JUN-98 23:58
operator : Gerald Ross
Simp Info : $200 \mathrm{ng} / \mathrm{ml}$ A 1660
Client Smp ID: $200 \mathrm{ng} / \mathrm{ml}$ A1660
Misc Info : $200 \mathrm{ng} / \mathrm{ml}$ A 1660
Inst ID: $g c 2 . i$
Comment :
Method : /chem/gc2.i/062298.b/rcrapcb.m
Meth Date : 24-Jun-1998 09:58 gar Quant Type: ESTD
Cal Date : 22-MAY-1998 20:13 Cal File: 028f0401.d
Als bottle: 1
Dil Factor: 1.000
Integrator: HP Genie
Target Version: 3.10
Compound Sublist: AR1660.sub Sample Matrix: WATER

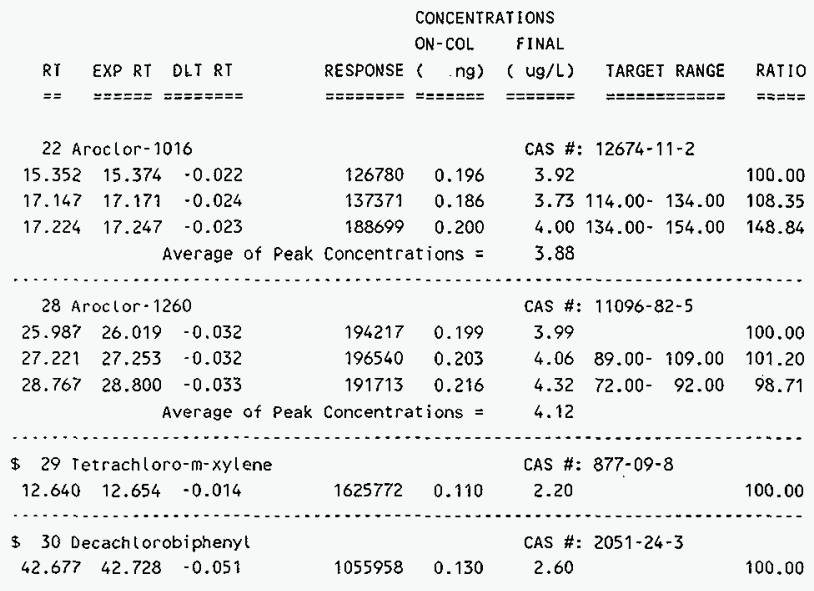


Data File: /chem/gc2.i/062298.b/029f1401.d

Report Date: 29-Jun-1998 13:43

$$
\begin{aligned}
& \text { 222-S Laboratory } \\
& \text { RECOVERY REPORT }
\end{aligned}
$$

Client Name:

Sample Matrix: LIQUID

Lab Smp Id: $200 \mathrm{ng} / \mathrm{ml}$ A1660

Level: LOW

Data Type: GC MULTI COMP

spikelist File: RESOLUTION.spk

Method File:/chem/gc2.i/062298.b/rcrapcb.m

\begin{tabular}{|c|c|c|c|c|}
\hline SURROGATE COMPOUND & $\begin{array}{l}\text { CONC } \\
\text { ADDED } \\
u g / L\end{array}$ & $\begin{array}{c}\text { CONC } \\
\text { RECOVERED } \\
\text { ug/L }\end{array}$ & RECOVERED & LIMITS \\
\hline $\begin{array}{l}\$ 29 \text { Tetrachloro-m-xyle } \\
\$ 30 \text { Decachlorobiphenyl }\end{array}$ & $\begin{array}{l}2.00 \\
2.00\end{array}$ & $\begin{array}{l}2.20 \\
2.60\end{array}$ & $\begin{array}{l}110.26 \\
129.80\end{array}$ & $\begin{array}{l}50-150 \\
50-150\end{array}$ \\
\hline
\end{tabular}

Misc Info: $200 \mathrm{ng} / \mathrm{ml} \mathrm{A} 1660$
Client SDG: 062298

Fraction: PEST

Client Smp ID: 200ng/ml A1660

operator: Gerald Ross

SampleType: SAMPLE

Quant Type: ESTD 
Data File:/chem/gc2.i/062298.b/029f1401.d

Report Date: 29-Jun-1998 13:43

\section{2-s Laboratory}

TARGET COMPOUNDS

Client Name:

Lab Smp Id: $200 \mathrm{ng} / \mathrm{ml}$ A1660

Sample Location:

Sample Date:

Sample Matrix: WATER

Analysis Type: PEST

Data Type: GC MULTI COMP

Misc Info: $200 \mathrm{ng} / \mathrm{ml}$ A 1660
Client SDG: 062298

Client Smp ID: $200 \mathrm{ng} / \mathrm{ml}$ A1660

Sample point:

Date Received:

Quant Type: ESTD

Level: LOW

operator: Gerald Ross

CONCENTRATION UNITS:

(ug/L or $u g / K G) ~ u g / L$

\begin{tabular}{|c|c|c|}
\hline 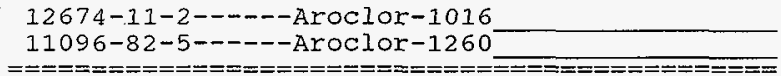 & $\begin{array}{r}3.88 \\
4.12\end{array}$ & \\
\hline $\begin{array}{l}877-09-8-\ldots-1 \\
2051-24-3 \cdots--0 \text { - Decach lorobiphenyl }\end{array}$ & $\begin{array}{r}2.20 \\
2.60\end{array}$ & $=-=$ \\
\hline
\end{tabular}


Data File; /chem/gc2.i/062298.b/029f1401.d

Date : 23-JuN-98 23:58

Client ID: $200 \mathrm{ng} / \mathrm{ml}$ A 1660

Sample Info: 200ng/ml A1660

Volume Injected (uL): 1,0

Colurin phase: Xti-5
Instrument: $\mathrm{gc2}+\mathrm{i}$

Operator: Gerald Ross

Coluan diameter: 0.25

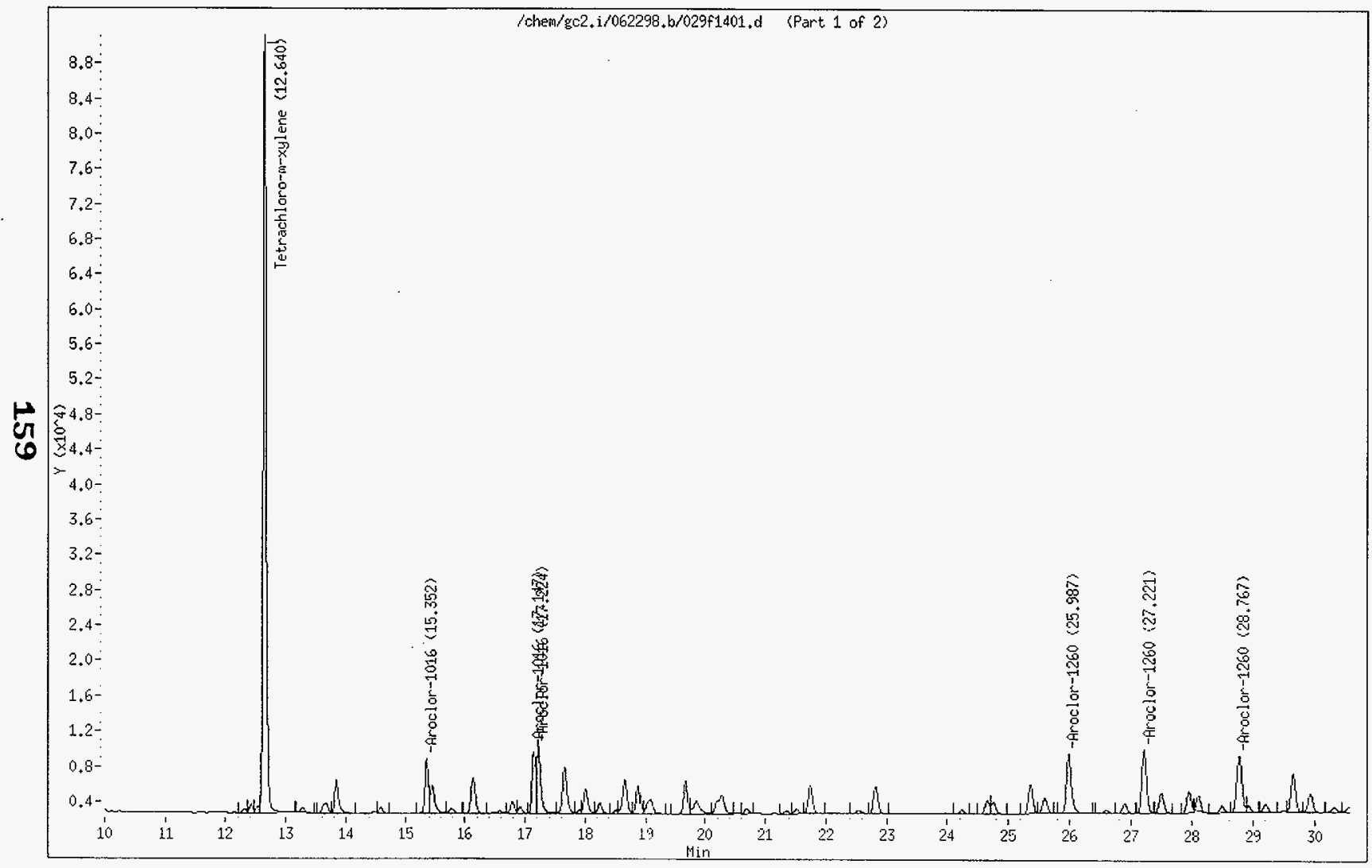




\section{DISTRIBUTION SHEET}

\begin{tabular}{|c|c|c|c|c|}
\hline \multirow{2}{*}{$\begin{array}{l}\text { To } \\
\text { Distribution }\end{array}$} & \multirow{2}{*}{\multicolumn{2}{|c|}{ Production Planning \& Control }} & \multicolumn{2}{|c|}{ Page 1 of 1} \\
\hline & & & \multicolumn{2}{|c|}{ Date: $07 / 29 / 98$} \\
\hline \multirow{2}{*}{\multicolumn{3}{|c|}{$\begin{array}{l}\text { Project Title/Work Order } \\
\text { HNF-SD-WM-DP-309, Rev. } 0 \text { "Final Report for Tank } 100 \text { Sump Sludge } \\
\text { (KON332) for Polychlorinated Biphenyl's (PCB)" }\end{array}$}} & \multicolumn{2}{|c|}{ EDT N0.: EDT-623162 } \\
\hline & & & \multicolumn{2}{|c|}{ ECN NO.: N/A } \\
\hline & MSIN & $\begin{array}{l}\text { Text With } \\
\text { all Attach }\end{array}$ & $\begin{array}{l}\text { EDT/ECN } \\
\text { ONLY }\end{array}$ & \\
\hline
\end{tabular}

Lockheed Martin Hanford Corp.
A. E. Young
R1-10
$x$

Lockheed Martin Services, Inc.

Central Files

A3-88 1

Los Alamos Technical Associates

M. T. Ellsworth

T6-12

$X^{*}$

Waste Management of Hanford. Inc.

R. K. Fuller

T6-50

\$3-30

K. L. Powel1

$\mathrm{T} 6-03$

S. M. Steele

T6-03

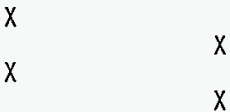

* Needs only releasing paperwork, not a copy of the released document. 